\title{
THE RESONANT BODY TRANSISTOR
}

\author{
A Dissertation \\ Presented to the Faculty of the Graduate School \\ of Cornell University \\ in Partial Fulfillment of the Requirements for the Degree of \\ Doctor of Philosophy
}

by

Dana Weinstein

August 2009 
(C) 2009 Dana Weinstein

ALL RIGHTS RESERVED 


\section{THE RESONANT BODY TRANSISTOR}

Dana Weinstein, Ph.D.

Cornell University 2009

With quality factors $(Q)$ often exceeding 10,000 , vibrating micromechanical resonators have emerged as leading candidates for on-chip versions of high- $Q$ resonators used in wireless communications systems, sensor networks, and clocking sources in microprocessors. However, extending the frequency of MEMS resonators generally entails scaling of resonator dimensions leading to increased motional impedance. In this dissertation, I introduce a new transduction mechanism using dielectric materials to improve performance and increase frequency of silicon-based RF acoustic resonators.

Traditionally, electrostatically transduced mechanical resonators have used air-gap capacitors for driving and sensing vibrations in the structure. To increase transduction efficiency, facilitate fabrication, and enable $\mathrm{GHz}$ frequencies of operation, it is desirable to replace air-gap transducers with dielectric films. In my doctoral work, I designed, fabricated, and demonstrated dielectrically transduced silicon bulk-mode resonators up to $6.2 \mathrm{GHz}$, marking the highest acoustic frequency measured in silicon to date. The concept of internal dielectric transduction is introduced, in which dielectric transducers are incorporated directly into the resonator body. With dielectric films positioned at points of maximum strain in the resonator, this transduction improves in efficiency with increasing frequency, enabling resonator scaling to previously unattainable frequencies. Using internal dielectric transduction, longitudinal-mode resonators exhibited the highest frequency-quality factor $(f . Q)$ product in silicon to date 
at $5.1 \times 10^{13} \mathrm{~s}^{-1}$.

These resonators were measured by capacitively driving and sensing acoustic vibrations in the device. However, capacitive detection often requires 3port scalar mixer measurement, complicating monolithic integration of the resonators with CMOS circuits. The internal dielectric bulk-mode resonators can be utilized in a 2-port configuration with capacitive drive and piezoresistive detection, in which carrier mobility is dynamically modulated by elastic waves in the resonator. Piezoresistive sensing of silicon-based dielectrically transduced resonators was demonstrated with $1.6 \%$ frequency tuning and control of piezoresistive transconductance $g_{m}$ by varying the current flowing through the device. Resonant frequency, determined by lithographically defined dimensions, was demonstrated over a wide frequency range. These degrees of freedom enable acoustic resonators spanning a large range of frequencies on a single chip, despite design restrictions of the CMOS process.

As we scale to deep sub-micron (DSM) technology, transistor cut-off frequencies increase, enabling the design of CMOS circuits for RF and mm-wave applications $>60 \mathrm{GHz}$. However, DSM transistors have limited gain and integrated passives demonstrate low $Q$, resulting in poor efficiency. A successful transition into DSM CMOS requires enhanced-gain and high-Q components operating at microwave frequencies. In this work, a merged NEMS-CMOS device is introduced that can function as a building block to enhance the performance of RF circuits. The device, termed the Resonant Body Transistor (RBT), consists of a field effect transistor embedded in the body of a high-frequency NEMS resonator implementing internal dielectric transduction. The results of this work indicate improved resonator performance with increased frequency, providing a means of scaling MEMS resonators to previously unattainable frequencies in 
silicon. With the transduction methods developed in this work, a hybrid NEMSCMOS RBT enables low-power, narrow-bandwidth low noise amplifier design for transceivers and low phase-noise oscillator arrays for clock generation and temperature sensing in microprocessors. 


\section{BIOGRAPHICAL SKETCH}

Dana Weinstein was born on September 25, 1982 to Eliahu and Orly Weinstein in Petach Tikva, Israel. Dana graduated from Francis Parker High School in San Diego, CA in 2000. She received Bachelor of Arts degrees in Physics and in Astrophysics from the University of California, Berkeley in 2004. During her time at Berkeley, she worked on ultrafast x-ray physics in Professor Roger Falcone's lab and at the Advanced Light Source at Lawrence Berkeley National Lab. She joined the Applied Physics department at Cornell University in 2004, working with Professor Sunil Bhave on RF MEMS resonators. Dana completed her Doctor of Philosophy in Applied Physics in 2009. She is currently an assistant professor in Electrical Engineering and Computer Science at the Massachusetts Institute of Technology. 
To my parents and sister for their patience and support. 


\section{ACKNOWLEDGEMENTS}

First and foremost, I wish to thank my research advisor, Professor Sunil Bhave, for introducing me to the field of MEMS and for his innovative ideas which helped launch my graduate research. I thank him for providing all the resources for which a graduate student could ever hope, and for his guidance, support, and friendship throughout the years. I sincerely thank my committee members, Professors Amit Lal, Paul McEuen, and Harold Craighead for their input and support in my research.

I am thankful to my fellow MEMS students for provoking discussions and collaborations on multiple fronts. From the OxideMEMS lab, I thank Hengky Chandrahalim, Lih-Feng Cheow, Chen Chen, Eugene Hwang, Suresh Sridaran, Tiffany Cheng, Susmita Bhandari, Laura Fegely, David Hutchison, and Wilson Zhou. From SonicMEMS, I wish to thank Shankar Radhakrishnan, Rajesh Duggirala, Ayesa Sinha, Kursad Araz, Serhan Ardanuc, Alper Bozkurt, Nori Yoshimizu, Steven Tin, Abhishek Ramkumar, Siva Prasad, Janet Shen, Kwame Amponsah, Larry Lu, and Yue Shi.

A significant portion of my doctoral work revolved around the fabrication

of MEMS devices. Of course, this would not have been possible without the Cornell Nanoscale Science and Technology Facility (CNF). I am grateful to the entire CNF staff for providing and maintaining one of the most comprehensive academic cleanrooms in the country. Their training and technical advice was invaluable to my graduate work. I would also like to thank David Fried, whose Independent-Gate FinFET process was the inspiration for my own fabrication process. I extend a special thanks to Alexander Gondarenko, Jae-Goo Lee, Carl Poitras, Rick Brown, Shankar Radhakrishnan, Gregory Simelgor, Quentin Diduck, and Steve Hickman for their help and advice on fabrication. I am end- 
lessly grateful to Abhishek Ramkumar for teaching me the ropes of microfabrication at the start of my graduate work, and for his superhuman generosity and patience both inside and outside the cleanroom. And of course, a very special thanks to Shahyaan Desai, who always had 40 solutions ready for every cleanroom problem, and around whom everything seemed to work. I am indebted to him for keeping me sane in the most trying of times.

Our collaborations with the Sony Nanotechnology Development Department in Japan was instrumental in my research. I am thankful to Dr. Koichi Ikeda, Shinya Morita and Shun Mitarai for their long hours and hard work, and for providing their expertise to the realization of high-yield dielectrically transduced resonators. Thanks to Dr. Hitoshi Tamada at Sony for making our collaborations possible.

I would also like to thank my collaborators and co-workers at IBM Watson Research Center, including Wilfried Haensch, Leland Chang, Jeffrey Sleight, Steven Laux, Steve Koester, Mike Guillorn, Lidija Sekaric, Oki Gunawan, Amlan Majumdar, David Greenberg, Xiao Hu Liu, and Paul Soloman. My experiences at IBM helped elucidate some of the key questions in device research.

My most heartful thanks go to my friends, both near and far, who remind me to have a little fun now and then and who have supported me throughout the years. And finally, I thank my family who has put up with me and my shenanigans for over a quarter century.

This work was funded by the DoD National Defence Science and Engineering Grant (NDSEG), Lockheed Martin, and Army Research Labs. 
TABLE OF CONTENTS

Biographical Sketch . . . . . . . . . . . . . . . iii

Dedication .................... iv

Acknowledgements . . . . . . . . . . . . . . . . v

Table of Contents . . . . . . . . . . . . . . . vii

List of Tables . . . . . . . . . . . . . . . . . ix

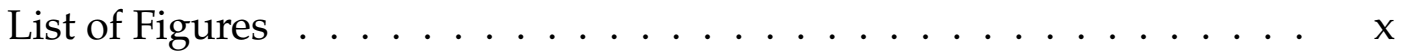

1 Introduction 1

1.1 Applications of Micro Electro-Mechanical Resonators . . . . . . . 1

1.2 Electrostatic Transduction Mechanisms . . . . . . . . . . . . . 2

1.2.1 Air-Gap Transduction . . . . . . . . . . . . . . . . . 2

1.2.2 External Dielectric Transduction . . . . . . . . . . . 7

1.2.3 Internal Dielectric Transduction . . . . . . . . . . . 8

2 MEMS Filters For Radio Communications 10

2.1 Radio Front End Design . . . . . . . . . . . . . . . . . 10

2.2 Thickness-Shear Mode Filters . . . . . . . . . . . . . . . . . . 12

2.2.1 Quarter-Wave Thickness Shear Bar Resonator . . . . . . . 13

2.2.2 Fabrication . . . . . . . . . . . . . . . . . . . . . . 19

2.2.3 Experimental Results . . . . . . . . . . . . . . . . . 20

2.2.4 Half-Wave Thickness Shear Bar Filters . . . . . . . . . . . . 23

2.3 Balanced-Unbalanced MEMS Filter . . . . . . . . . . . . . . . . . . 25

2.4 2D Resonator Array For MEMS Filters . . . . . . . . . . . . . . . 34

2.4 .1 Theory . . . . . . . . . . . . . . . . . 34

2.4.2 Experimental Setup . . . . . . . . . . . . . . . . . . . 38

2.4 .3 Experimental Results . . . . . . . . . . . . . . . . . . . . . . 41

2.4 .4 Conclusion . . . . . . . . . . . . . . . . . 45

3 Internal Dielectric Transduction: Theory 47

3.1 Motivation . . . . . . . . . . . . . . . . . . 47

3.2 Analysis: Capacitive Drive And Sense . . . . . . . . . . . . . 48

3.3 Analysis: Electrostriction . . . . . . . . . . . . . . . . . . 54

$4 \quad$ Internal Dielectric Transduction: Fabrication 57

4.1 IDT Devices At The Cornell Nanoscale Facility . . . . . . . . . . . 57

4.2 IDT Devices At The Sony Nanotechnology Development Department . . . . . . . . . . . . . . . . . . 59

5 Internal Dielectric Transduction: Measured Results 63

5.1 Capacitive Drive And Sense . . . . . . . . . . . . . . . . 63

5.2 Capacitive Drive And Piezoresistive Sense . . . . . . . . . . . 69 
6 The Resonant Body Transistor: Theory 79

6.1 Motivation .................... 79

6.2 Principle Of Operation . . . . . . . . . . . . . . . . . . . . . 80

6.3 Noise Analysis . . . . . . . . . . . . . . . . . . 85

6.3.1 Electrostatic Drive Noise . . . . . . . . . . . . . . . 85

6.3.2 Mechanical Thermal Noise . . . . . . . . . . . . . . 89

6.3 .3 Sensing Noise . . . . . . . . . . . . . . . . 91

6.3.4 Conclusion ................... 96

7 The Resonant Body Transistor: Fabrication 98

7.1 ALN: Alignment Marks . . . . . . . . . . . . . . . . . . . 99

7.2 RX: Single-Crystal Silicon Fin And Inner Resonator . . . . . . . . 100

7.3 PC: Polysilicon Gate And Outer Resonator . . . . . . . . . . . . . 101

7.4 Ion Implant Of Device Layer . . . . . . . . . . . . . . . . . . . . . . . . . . . . . . . . . . . . . . . . . .

7.5 Metallization ................... 106

7.5.1 SUB And CA: FOX Cladding And Contact Vias . . . . . . 106

7.5.2 M1: Nickel Silicide . . . . . . . . . . . . . . . . . 108

7.5.3 M1: Nickel Metallization . . . . . . . . . . . . . . . 109

7.6 REL: Release . . . . . . . . . . . . . . . . . . . . . 110

8 The Resonant Body Transistor: Measured Results 113

8.1 Measurement Setup . . . . . . . . . . . . . . . . . . . . 113

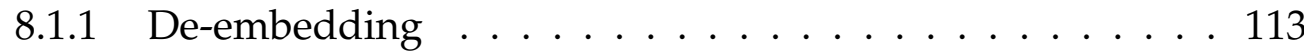

8.1 .2 RF Measurement . . . . . . . . . . . . . . 116

8.2 Experimental Results . . . . . . . . . . . . . 117

9 Future Directions $\quad 121$

9.1 Unreleased Resonant Body Transistors . . . . . . . . . . . . . . . . 121

9.2 The Resonant Body Oscillator . . . . . . . . . . . . . . . . . . . . . . . . . . . . 122

9.3 Synchronization . . . . . . . . . . . . . . . 123

$\begin{array}{ll}\text { A Dielectric vs. Piezoelectric Transduction } & 125\end{array}$

A.1 Dielectric Film Actuation . . . . . . . . . . . . . . . . . 125

A.1.1 Beam Shape Due To Dielectric Stress Transfer . . . . . . . . 125

A.1.2 Sensing And Motional Impedance . . . . . . . . . . . . 128

A.2 Piezoelectric Film Actuation . . . . . . . . . . . . . . . . . . . 131

A.2.1 Beam Shape Due To Piezoelectric Stress Transfer . . . . . . 131

A.2.2 Sensing And Motional Impedance . . . . . . . . . . . . . 132

A.3 Comparison Of Actuation Transfer Functions . . . . . . . . . . . . 133

B RBT Fabrication Process $\quad 135$

$\begin{array}{ll}\text { Bibliography } & 142\end{array}$ 


\section{LIST OF TABLES}

2.1 Summary Of $50 \Omega$ Terminated Filter Performance . . . . . . . . 45

6.1 Symbol Definition . . . . . . . . . . . . . . . . . 81

6.2 Assumed Constants . . . . . . . . . . . . . . . . . . . 82

6.3 Johnson Noise For Resonator Drive . . . . . . . . . . . . . 87

6.4 Cutoff Frequency At Drive Point . . . . . . . . . . . . . . 88

B.1 RBT Fabricatin Process . . . . . . . . . . . . . . . . . . . . . . 135 


\section{LIST OF FIGURES}

1.1 Schematic of air-gap electrostatic transduction in a longitudinal bar resonator. An equivalent circuit can be established for the resonator with an RLC circuit. . . . . . . . . . . . . . . . . . 4

2.1 Two-pole mechanical filter, comprising two mass-springdamper systems joined by a coupling spring. . . . . . . . . . . .

2.2 Driving mechanism for shear acoustic vibrations in a resonator using lateral dielectric transduction. . . . . . . . . . . . . . 13

2.3 Sensing mechanism for shear acoustic vibrations in a resonator using lateral dielectric transduction. . . . . . . . . . . . . . 14

2.4 X-displacement contour plot from Ansys simulation of the symmetric quarter-wave thickness shear mode. . . . . . . . . . . . . 15

2.5 Unreleased thickness shear bar diagram. . . . . . . . . . . . 16

2.6 SOI-based micro-fabrication process for lateral dielectrically transduced resonators. . . . . . . . . . . . . . . . . . . . 19

2.7 SEM of a silicon nitride-on-silicon unreleased bar resonator. Measured transmission of the thickness shear mode of the unreleased resonator in air. . . . . . . . . . . . . . . . . . . 20

2.8 Measured transmission of the shear mode of the partially released resonator in air. The reduction in anchor size increased the quality factor almost three-fold. . . . . . . . . . . . . . . 22

2.9 (left) Tuning schematic for the partially released bar resonator, applying a bias voltage to bend the resonator perpendicular to the resonant direction of motion. (right) Resonant frequency vs. tuning voltage $V_{P}$ of the bar resonator. A tuning range of about $5 \mathrm{MHz}$ is observed. . . . . . . . . . . . . . . .

2.10 ANSYS contour plot of the symmetric half-wave thickness shear mode. This mode causes the tether suspension at the nodal plane to move along the Z-axis, as indicated by the down arrows. . . .

2.11 ANSYS simulation of length $(L)$ vs. resonant frequency of the 3.2 $\mu m$ thick half-wave shear mode resonator. The pure shear mode resonant frequency of the bar is $844 \mathrm{MHz} . \ldots \ldots . . \ldots 26$

2.12 (a) Scanning electron micrograph of a hafnium dioxide-onsilicon fully released bar resonator. (b) Scanning electron micrograph of the $30 \mathrm{~nm}$ hafnium dioxide layer on the top of the silicon resonator. . . . . . . . . . . . . . . .

2.13 Measured transmission response of a half-wave thickness shear mode resonator in air. The $f . Q$ product of the resonator is $6.2 \times 10^{12} .28$

2.14 Scanning electron micrograph of a mechanically coupled shear arc filter. . . . . . . . . . . . . . . . . . 28 
2.15 Transmission response of the mechanically coupled arc filter. The arc filter characteristics show improved stop-band rejection and $20 \mathrm{~dB}$ shape factor compared to the electrically coupled filters. 29

2.16 Schematic of a radio front end with in-line RF switch, filter, and balun transformer. These three components can be replaced with a single-ended to differential MEMS filter. . . . . . . . . . . . .

2.17 Driving mechanism for longitudinal acoustic vibrations in a resonator using lateral dielectric transduction. . . . . . . . . . . . 30

2.18 Sensing mechanism for longitudinal acoustic vibrations in a resonator using lateral dielectric transduction. . . . . . . . . . . .

2.19 Scanning electron micrograph of single-ended to differential MEMS filter. The antenna signal drives resonance in a breathingmode disk, which couples mechanically to a wine glass ring resonator. . . . . . . . . . . . . . . . . . . .

2.20 Equivalent circuit for single-ended to differential MEMS filter comprised of two types resonators coupled with a mechanical spring. . . . . . . . . . . . . . . . . .

2.21 Measured frequency response of a $425 \mathrm{MHz}$ single-ended to differential filter. . . . . . . . . . . . . . . . . . 34

2.22 Measured frequency response of a $910 \mathrm{MHz}$ single-ended to differential filter. . . . . . . . . . . . . . . . . . . .

2.23 Broad frequency sweep of the $910 \mathrm{MHz}$ filter, showing lower frequency spurious modes suppressed below $-40 \mathrm{~dB}$. . . . . . . . . 36

2.24 Switching capabilities of the MEMS filter. Applying a bias voltage across the dielectric transducer passes signals in the targeted passband. Switching that bias voltage off allows no signal to pass. 37

2.25 2D mechanical coupling configuration under investigation to reduce effective resonator variations. Here, the coupling stiffness

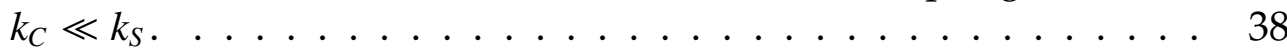

2.26 Scanning electron micrographs of (a) a 1D 4-pole filter, (b) 4 electrically summed 1D 4-pole filters, (c) a 4x4 array of resonators, coupled weakly in both directions, and (d) a $4 \times 4$ array of resonators, coupled weakly in one direction and strongly in the other. 39

2.27 $50 \Omega$ terminated $S_{21}$ transmission response of a single wine glass

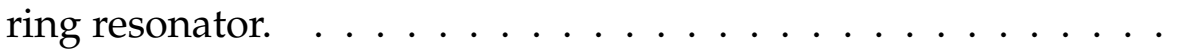

$2.2850 \Omega$ terminated $S_{21}$ transmission plot of (a)1D 4-pole filter, (b) comparison of 1D filter with 4 electrically summed 1D filters, (c) comparison of 1D filter with $4 \times 4$ 2D weakly coupled array, and (d) comparison of 1D filter with 4x4 2D strongly coupled array. . 
3.1 (a) Schematic of dielectrically transduced free-free longitudinal bulk mode resonator. The dielectric films are incorporated into the resonator, driving and sensing electrostatically. (b) Cross section of bar resonator. A bias voltage $V_{D C}$ is applied to the resonator. An AC voltage $v_{\text {in }}$ on one end drives resonance, while an output current $i_{\text {out }}$ is measured at the other. The normalized amplitudes of the 3rd and 9th longitudinal mode harmonics are displayed. . . . . . . . . . . . . . . . .

3.2 $R_{X}$ as a function of fractional dielectric position $\left|\frac{2 d}{L}\right|$ for the $3 \mathrm{rd}$ and 9th harmonic longitudinal (width-extensional) modes of a $8.5 \mu \mathrm{m}$ long bar. $\mathrm{g}=15 \mathrm{~nm}$, resonator thickness is $2.5 \mu \mathrm{m}$, width is $40 \mu \mathrm{m}, V_{D C}=10 \mathrm{~V}, \varepsilon_{f}=7 \varepsilon_{0}$, and an $f Q$ product of $5 \times 10^{13}$ is assumed. . . . . . . . . . . . . . . .

3.3 $R_{X}$ scaling with frequency, normalized to the cross-sectional area of the resonator. $\mathrm{g}=15 \mathrm{~nm}, V_{D C}=10 \mathrm{~V}$, and $\varepsilon_{f}=7 \varepsilon_{0}$. The dielectric films are placed at maximum strain. An $f . Q$ product of $5 \times 10^{13}$ is assumed. . . . . . . . . . . . .

4.1 Micro-fabrication process for internal dielectric transduced resonators. . . . . . . . . . . . . . . . . . .

4.2 Left: Modal analysis of 3rd harmonic resonant mode shape of the bar resonator, simulated in Ansys. Right: Scanning Electron Micrograph of a dielectrically transduced silicon bar resonator (8.5 $\mu m$ long $\times 40 \mu m$ wide $\times 2.5 \mu m$ tall). The inset image shows the thin gap between the polysilicon and single crystal regions of the resonator. . . . . . . . . . . . . . . . . . . . .

4.3 An abbreviated illustration of the Sony-based surface micromachining process for dielectrically transduced resonators. The longitudinal resonators are comprised of Polysilicon with $15 \mathrm{~nm}$ Silicon Nitride transducers. . . . . . . . . . . . . . . . . . . .

4.4 Left: Scanning Electron Micrograph of an all-Polysilicon dielectrically transduced silicon bar resonator $(8.5 \mu \mathrm{m}$ long $\times 40 \mu \mathrm{m}$ wide $\times 2 \mu \mathrm{m}$ tall). The inset image shows the $15 \mathrm{~nm}$ Nitride transducer sandwiched between two Polysilicon regions of the

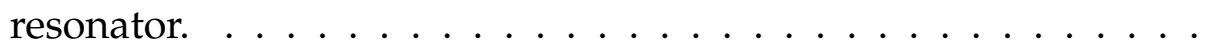

5.1 Schematic of scalar mixer measurement of the 3-port MEMS resonator. The resonator (DUT) acts as a mixer for the input RF and $\mathrm{LO}$ signals. The resonance is detected at RF - $\mathrm{LO}$, thus preventing effects of feed-through capacitance in the transmitted frequency response . . .................. 
5.2 Measured frequency response of 3rd and 9th harmonic resonance of the silicon bar resonator. The grey traces $(0 \mathrm{~V})$ indicate the LO leakage of the device. Applying a $5 \mathrm{~V}$ bias excites acoustic resonance, shown in black. The 9 th harmonic exhibits a $9.8 \mathrm{~dB}$ absolute improvement in signal strength over the 3rd harmonic mode, with a $Q$ increase of $6.6 \times$. Normalizing the resonant peaks by $Q$, we extract a $2 \mathrm{~dB}$ enhancement of transduction efficiency in the $4.5 \mathrm{GHz}$ resonance relative to the $1.5 \mathrm{GHz}$ resonant mode.

5.3 Motional impedance vs. position of dielectric transducer in 3rd and 9 th harmonics of 8 different resonators. Selective positioning of dielectric can excite \# 1 only 9th harmonic at $4.72 \mathrm{GHz}$, \# 2 only 3rd harmonic at $1.55 \mathrm{GHz}$, and \# 3 both 3rd and 9th harmonics. . . . . . . . . . . . . . . . .

5.4 Measured frequency response of 3rd and 9th harmonics of two bar resonators. The 9th harmonic at $6.2 \mathrm{GHz}$ marks the highest frequency measured in silicon to date. . . . . . . . . . . . . .

5.5 Motional impedance scaling with resonant frequency. Comparison of resonators with varying harmonics $\mathrm{n}$ and quality factors (Q) necessitates normalization of $\mathrm{Rx}$ as shown (normalization factor $Q_{0}=5000$ ). As predicted by theory, Rx improves with increasing frequency. . . . . . . . . . . . . . .

5.6 Contour plot of current density in the resonator. Current flow is uniform through most of the resonator body. Current nonuniformity at input and output of the resonator is the primary source of piezoresistive signal. . . . . . . . . . . . . . .

5.7 Spatial distribution of piezoresistive signal. The symmetry of the strain in the resonator results in cancellation of the signal in the majority of the body. The piezoresistance peaks near the routing beams generate the detected resonant signal. . . . . . . . . . 72

5.8 Schematic of measurement setup for internal dielectric actuation and piezoresistive detection. . . . . . . . . . . . . 73

5.9 Measured frequency response of transconductance gm of the resonator for varying drain currents. . . . . . . . . . . . . . . 75

5.10 Measured resonant frequency scaling with increasing drain current. Resistive heating thermally expands the resonator, decreasing resonant frequency with increasing internal temperature . . 76

5.11 Measured electromechanical $Q_{e m}$ with increasing drain current. . 76

5.12 Experimental resonator transconductance $g_{m}$ scaling with increasing drain current. . . . . . . . . . . . . . . . 77

6.1 (left) Longitudinal bar resonator driven and sensed using Internal Dielectric Transduction. (right) A Resonant Body Transistor (RBT) driven with Internal Dielectric Transduction. . . . . . . . . 80

6.2 Principle of operation for an internal dielectrically driven RBT. . 83 
6.3 Frequency scaling comparison of motional impedance $R_{X}$ for a capacitive resonator and an RBT. . . . . . . . . . . . . .

6.4 Relative performance of a capacitive resonator to the RBT scaling with frequency. . . . . . . . . . . . . . . . 86

6.5 Frequency scaling of the motional admittance of the RBT. . . . . 86

6.6 Schematic of measurement setup for a capacitively-sensed IDT resonator. The resonator body is biased to $V_{D C}$, while an RF excitation across one dielectric transducer drives resonance. An RF current is detected across the second dielectric transducer. . . . .

6.7 Flicker noise current in an IDT sensed using the MOSFET configuration. The pA amplitude of the noise current at frequencies of interest imply that flicker noise does not dominate the noise in the system.

7.1 (left) Layout of die comprising RBTs ranging from 10 to $20 \mathrm{GHz}$ along (100) and (110) directions and split-gate FinFETs along (110) direction. (right) Zoomed in view of a single device showing metal pads and routing to the device. . . . . . . . . . . 98

7.2 (left) Hard oxide mask (RX) to pattern the SOI device layer. (right) Device layer with remaining hard oxide mask etched in an ICP RIE chlorine etch. . . . . . . . . . . . . . . . . . . 101

7.3 (left) Polysilicon planarization with an etch stop on the hard oxide mask used for device layer patterning. (right) Hard oxide mask (PG) patterning for poly gate definition and ion implant mask. . . . . . . . . . . . . . . . . . . . . . . . . 103

7.4 (left) Polysilicon etch in an ICP RIE chlorine etch to define gates and resonator body. (right) Removal of RX hard oxide mask for ion implant of device layer. . . . . . . . . . . . . . . . . . . . . 104

7.5 (left) Contact holes etched into field oxide for electrical connection to the device. (right) Nickel silicide and metallization using conformal e-beam evaporation and liftoff. . . . . . . . . . . 107

7.6 XPS scan of a Ni silicide sample indicating no Au contamination or other impurities during silicide formation. . . . . . . . . . . 110

7.7 (left) Released FinFET showing metal contacts and release window. (right) Released RBT and FinFET. The darker region in silicon indicates the undoped active area of the device. . . . . . . . . 112

8.1 De-embedding structures fabricated on-chip to subtract parasitic capacitance and inductance of the probe pads and routing, lowering the measurement floor. . . . . . . . . . . . . . . . . 114

8.2 Measurement setup for the resonant body transistor. . . . . . 116 
8.3 De-embedded transconductance measurement of a Split-Gate FinFET with both gates tied together and biased into strong inversion. The fin width is $50 \mathrm{~nm}$ and gate length is $500 \mathrm{~nm}$, with a $\mathrm{W} / \mathrm{L}$ ratio of $\sim 0.4 \ldots \ldots \ldots \ldots$. . . . . . . . . . . . 118

8.4 Measured RBT frequency response at its 3rd harmonic longitudinal resonance. . . . . . . . . . . . . . . . . . . . . . . . . 119

9.1 Cross section of CMOS stack showing acoustically excited Resonant Body Transistors and patterning of silicon, insulation, and metal layers to localize acoustic resonance. . . . . . . . . . . . 122

9.2 Schematic of Resonant Body Oscillator comprising an RBT. Acoustic resonance in the transistor will enable low-power, low phase noise oscillators at $\mathrm{GHz}$ frequencies with $<1 \mu \mathrm{m}^{2}$ footprint. 123

9.3 Acoustic synchronization of an array of Resonant Body Oscillators through mechanical coupling between adjacent Resonant Body Transistors. Coupling can be induced from vibrations through the substrate or insulation or using mechanical coupling springs. . . . . . . . . . . . . . . . . . . . . . . 124 
CHAPTER 1

INTRODUCTION

\subsection{Applications of Micro Electro-Mechanical Resonators}

Semiconductor electromechanical resonators, with quality factors $(Q)$ often exceeding 10,000, provide a low-power, small footprint, CMOS-compatible alternative to various electrical components in wireless communication and signal processing. As the communication industry moves towards quad-band and 7band technology, there is a growing demand for light-weight, low-power, compact cell phones that operate at the global range of frequencies. Currently, radio front ends require 10-15 large filters fabricated on different substrates using conventional Surface Acoustic Wave (SAW) or Film Bulk Acoustic Resonator (FBAR) technology. However, filter banks composed of high- $Q$ micromechanical filters can be fabricated on-chip in silicon, reducing size, weight, cost, and power in radio communications.

Silicon micromechanical resonators also have promising applications in $\mathrm{mi}-$ croprocessor technology. As microprocessors scale to higher frequencies of operation and towards multi-core systems, clocking precision and synchronization at every register becomes increasingly important. High- $Q$, small footprint, CMOS-integrated mechanical resonators can provide synchronized clocking arrays in high-performance microprocessors with reduced power, jitter, and skew. 


\subsection{Electrostatic Transduction Mechanisms}

Within the realm of Micro Electro-Mechanical Systems (MEMS), there exist many species of mechanically resonant devices accumulated over more than half a century of research and development. Two of the primary classifications for these resonators are (1) the resonant eigenmode implemented in the device (e.g. flexural, longitudinal, shear, torsional, compound mode) and (2) the transduction mechanism used to excite and detect motion. The term transduction, as it pertains to MEMS resonators, means the transfer of energy from one domain to another. For example, an electrostatic transducer converts an electrical signal into a mechanical vibration, or vice versa.

A variety of transduction mechanisms can be used to drive and sense MEMS resonators, each with its own advantages and disadvantages. Popular transducers include electrostatic, piezoelectric, thermal, optical, opto-mechanical, and magnetic transducers, to name a few. This work focuses on electrostatic transduction, in which an electrical signal is converted into mechanical vibrations using an electrostatic (capacitive) force. The induced mechanical energy is then transferred back into an electrical output through a time-varying capacitance.

\subsubsection{Air-Gap Transduction}

One of the most notable inventions in the field of MEMS resonators is the combdrive resonator in 1989 by Bill Tang and Roger Howe [1]. The comb-drive is comprised of a mass freely suspended by flexural beams anchored to a substrate. The suspended mass includes rows of fingers (comb) forming a large capacitance when the fingers are interdigitated with an anchored comb. The 
air-gap capacitance of the comb-drive generates a force used to accelerate the mass back and forth, resonating at a frequency corresponding to the comb-drive mass and the stiffness of the flexural beam suspension. This device popularized MEMS resonators and Polysilicon surface micromachining in the early 90s, launching a generation of air-gap electrostatically transduced resonators.

More recently, the push to higher frequencies of operation has prompted the development of bulk-mode resonators using air-gap transduction [2, 3]. The principle of air-gap electrostatic transduction in these bulk-mode devices is as follows.

Let us take the example of a longitudinal bar resonator driven and sensed using two air-gap capacitors, as shown in Figure 1.1. A bias voltage $V_{D C}$ is first applied to the resonator body. A driving AC voltage $v_{\text {in }}$ applied to the drive electrode generates a force on the resonator surface

$$
\begin{aligned}
F_{\text {cap }} & =\frac{d U_{\text {cap }}}{d x} \\
& =\frac{d}{d x}\left(\frac{1}{2} C_{\text {drive }} V^{2}\right) \\
& =\frac{1}{2} V^{2} \frac{\partial C}{\partial x} \\
& =\frac{1}{2}\left(V_{D C}+v_{i n} e^{i \omega t}\right)^{2} \frac{\partial}{\partial x}\left(\frac{\varepsilon_{0} A_{c a p}}{g+x}\right) \\
& \approx \frac{\varepsilon_{0} A_{c a p}}{g^{2}}\left(V_{D C}^{2}+2 V_{D C} v_{i n} e^{i \omega t}+v_{i n}^{2} e^{i 2 \omega t}\right)
\end{aligned}
$$

where $U_{c a p}$ is the energy stored in the capacitor, $A_{c a p}$ is the area of the capacitor, $g$ is the capacitive gap, $\varepsilon_{0}$ is the permittivity of free space, and $\omega$ is the frequency of the driving signal. Equation 1.1 dictates that the electrostatic drive results in a capacitive force at DC, $\omega$, and $2 \omega$. The DC component of the force generates a constant displacement of the bar resonator, not resulting in any resonant vibrations. In the case of the bar resonator in Figure 1.1, the drive and sense 
capacitors generate opposing DC forces which cancel each other out. The force at $2 \omega$ is proportional to $v_{i n}^{2}$. Assuming $v_{i n} \ll V_{D C}$, this term is negligible relative to the force at $\omega$. Therefore, the only force term relevant to electrostatic transduction in this case is

$$
f_{c a p, \omega}=V_{D C} v_{i n} \frac{\varepsilon_{0} A_{c a p}}{g^{2}} .
$$
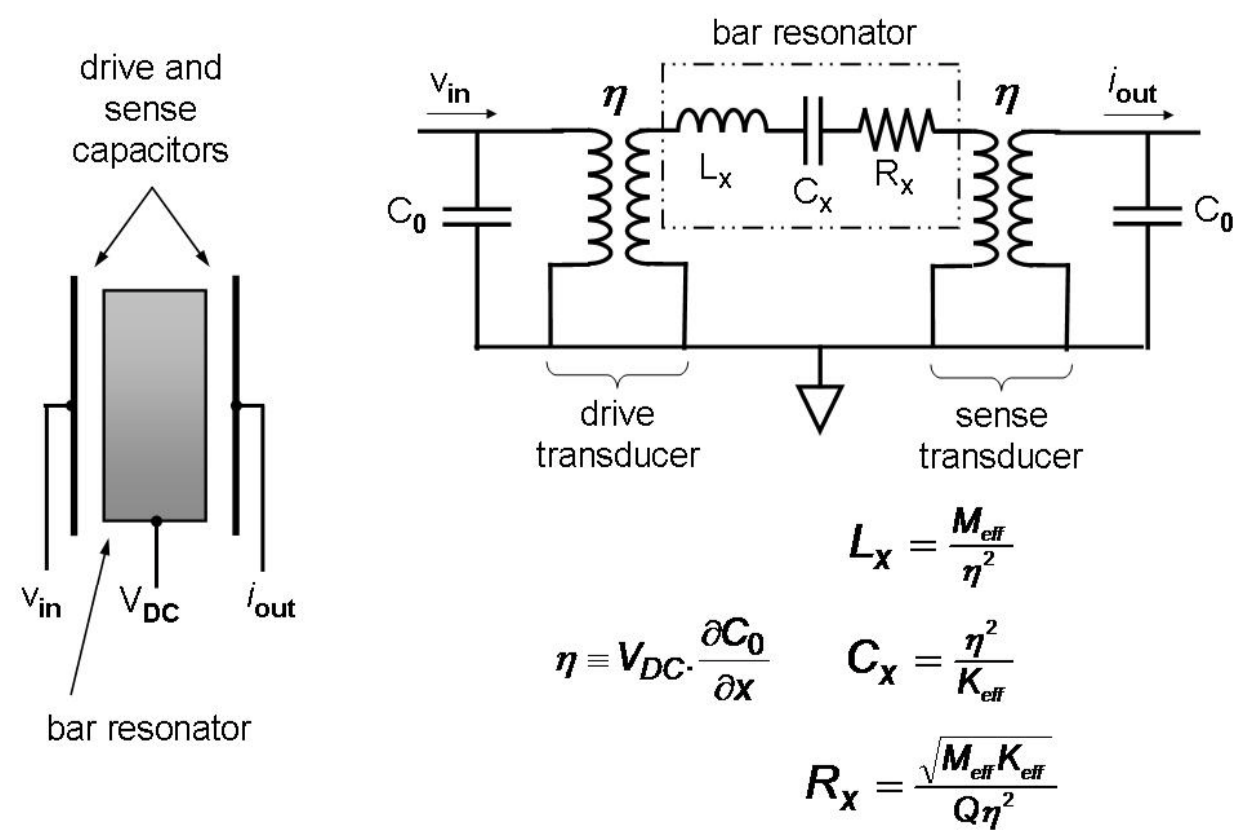

Figure 1.1: Schematic of air-gap electrostatic transduction in a longitudinal bar resonator. An equivalent circuit can be established for the resonator with an RLC circuit.

The displacement of the $n$th harmonic longitudinal mode of a bar of length $L$ is given by

$$
u(x)=U_{0} \sin \left(\frac{n \pi}{L} x\right)
$$


where $U_{0}$ is the amplitude of displacement. In this example, we wish to excite the first harmonic $(n=1)$ of a bar with a Young's modulus of $Y$ and a mass density of $\rho$. With the acoustic velocity in the bar given by $c_{s}=\sqrt{Y / \rho}$, the resonant frequency of the first harmonic is

$$
f_{\text {res }}=\frac{1}{2 L} \sqrt{\frac{Y}{\rho}} .
$$

To obtain an equivalent RLC circuit as described in Figure 1.1, we must first calculate the effective mass $M_{e f f}$ and stiffness $K_{\text {eff }}$ corresponding to the first longitudinal harmonic of the bar. Assuming a lumped mass-spring-damper model for the bar resonator, the effective mass can be determined by equating the kinetic energy of the lumped model to that of the distributed system.

$$
\begin{aligned}
\left.\frac{1}{2} M_{e f f} v\right|_{x=L / 2} ^{2} & =\frac{1}{2} \rho A_{\text {cap }} \int_{-L / 2}^{L / 2} v(x)^{2} d x \\
M_{e f f} & =\frac{\rho A_{\text {cap }}}{u(L / 2)^{2}} \int_{-L / 2}^{L / 2} u(x)^{2} d x \\
& =\left.\rho A_{\text {cap }}\left(\frac{x}{2}-\frac{L \sin \left(\frac{2 \pi x}{L}\right)}{4 \pi}\right)\right|_{x=L / 2} \\
& =\frac{1}{2} \rho A_{\text {cap }} L .
\end{aligned}
$$

The results of Equation 1.5 are in fact identical for all harmonics of longitudinal vibrations in the bar. Given the effective mass and the resonant frequency, the effective stiffness of the resonant mode can be determined:

$$
K_{\text {eff }}=\frac{\pi^{2}}{2} \frac{A_{\text {cap }} Y}{L} .
$$


Since we treat the resonator as a lumped model with mass $M_{e f f}$ concentrated at a position $x=L / 2$, the amplitude of vibrations of the resonant mode $U_{0}$ can now be found simply using Hooke's Law $U_{0}=f_{\text {cap, } \omega} / K_{\text {eff }}$ and Equations 1.6 and 1.2

$$
U_{0}=\frac{2 L v_{i n} V_{D C} \varepsilon_{0}}{g^{2} \pi^{2} Y}
$$

This harmonic displacement results in a modulation of the sense capacitor of the resonator, resulting in an $\mathrm{AC}$ current at the output

$$
\begin{aligned}
i_{\text {out }} & =\frac{d Q}{d t}=V_{D C} \frac{d C_{0}}{d t}=V_{D C} \frac{d C_{0}}{d x} \frac{d x}{d t} \\
& =V_{D C} \frac{\varepsilon_{0} A_{\text {cap }}}{g^{2}} \omega_{\text {res }} U_{0} \\
& =\frac{2 A_{\text {cap }} V_{D C}^{2} \varepsilon_{0}^{2} v_{\text {in }}}{\pi g^{4} \sqrt{Y \rho}}
\end{aligned}
$$

The motional impedance $R_{X} \equiv v_{\text {in }} / i_{\text {out }}$ of the resonator can now be determined from Equation 1.8. The motional impedance is one of the most important figures of merit in resonator design, and will be referred to frequently throughout this work. $R_{X}$ defines the resonator's efficiency in converting transferring energy from the electrical to the mechanical domain and converting that mechanical energy back into an electrical signal. A small motional impedance is always desired, resulting in a large output current for a small input signal. Using Equation 1.8 and the definition of the motional impedance, we find

$$
R_{X}=\frac{\pi g^{4} \sqrt{Y \rho}}{2 Q A_{\text {cap }} V_{D C}^{2} \varepsilon_{0}^{2}}
$$

An alternate method commonly used to determine the motional impedance 
is to use Equations 1.5 and 1.6 with those of Figure 1.1. The equivalent circuit elements are

$$
\begin{gathered}
\eta^{2}=V_{D C} \frac{\varepsilon_{0} A_{\text {cap }}}{g^{2}} \\
L_{X}=\frac{g^{4} L \rho}{2 A_{\text {cap }} V_{D C}^{2} \varepsilon_{0}^{2}} \\
C_{X}=\frac{2 A L V_{D \varepsilon_{0}^{2}}^{2}}{g^{4} \pi^{2} Y} \\
R_{X}=\frac{\pi g^{4} \sqrt{Y \rho}}{2 Q A_{\text {cap }} V_{D C}^{2} \varepsilon_{0}^{2}}
\end{gathered}
$$

It can be readily seen from Equations 1.9 and 1.13 that both methods result in the same motional impedance. However, the equivalent circuit method may be difficult to implement in more complex distributed systems which are not so readily formed into lumped models. In general, it is therefore advisable to formulate $R_{X}$ based on the force applied to the resonator and the current detected at the output.

\subsubsection{External Dielectric Transduction}

Based on the results of Equation 1.9, one of the easiest ways to improve the motional impedance of an electrostatically transduced resonator is to increase the permittivity within the drive and sense capacitors, as $R_{X} \propto 1 / \varepsilon^{2}$. This can be achieved by filling the air-gap with a dielectric material. In 1989, Bouwstra et al demonstrated a cantilever beam resonator at $2 \mathrm{kHz}$ transduced with a thin Silicon Nitride film deposited on top of the cantilever [4]. The principle of dielectric

electrostatic transduction was extended to bulk-mode resonators by Bhave and Howe in 2004 [5] and demonstrated in a $60 \mathrm{MHz}$ disk resonator [6], a $120 \mathrm{MHz}$ 
longitudinal bar resonator [7], and a $713 \mathrm{MHz}$ thickness shear resonator [8].

These devices can all be categorized as using external dielectric transducers, as the capacitive transducers are designed adjacent to or on top of the resonator. For instance, the disk resonator in [6] is identical to its air-gap counterpart apart from the dielectric film introduced into the air-gaps. In the case of the air-gap disk, the perimeter of the resonator is subject to free boundary conditions whereby all acoustic energy incident on the disk edge is reflected back. To excite the same resonant mode in the dielecrically transduced disk, only the reflected component of acoustic waves at the interface of the disk and the dielectric contribute to the resonant mode. Any acoustic waves transmitted through the dielectric film into the electrodes corresponds to lost energy and inefficiency in transduction.

At low frequencies $(<1 \mathrm{GHz})$, the reduction in $R_{X}$ due to the increased permittivity in the drive and sense capacitors outweighs the loss in transduction efficiency due to imperfect boundary conditions as discussed above. However, as resonant frequency increases, the efficiency of external dielectric transduction drops [9]. In the multi-GHz frequency domain, a novel transduction mechanism, termed internal dielectric transduction (IDT) provides the benefits of high permittivity capacitive transducers without the losses observed in external dielectric transduction.

\subsubsection{Internal Dielectric Transduction}

The primary difference between internal and external dielectric transduction is the incorporation of the dielectric transducer into the resonator body (and into the resonant mode shape) in IDT devices. External transduction implements di- 
electric electrostatic transducers at the resonator boundaries to drive and sense motion, thereby disturbing to boundary conditions necessary to define the resonant acoustic standing waves in the device. Alternately, IDT drives and senses acoustic vibrations from dielectric films within the resonator body.

Unlike external transducers, the internal drive and sense afforded by IDT allow for the resonator boundary to remain free, as in the case of air-gap transducers. A complete analysis of IDT is presented in Chapter 3, showing optimal IDT design with improved transduction efficiency with increasing frequency. 


\section{CHAPTER 2}

\section{MEMS FILTERS FOR RADIO COMMUNICATIONS}

\subsection{Radio Front End Design}

Numerous applications in cellular transceivers and sensor networks have driven the development of on-chip, high- $Q$ MEMS resonators and filters to replace existing off-chip SAW and ceramic resonator technologies. Receivers operating in the ISM band (902 - $928 \mathrm{MHz}$ ) have narrow channels and are susceptible to nearby strong interferers. Channel-select filtering requires small bandwidth, good stop-band rejection, and excellent shape factor to filter out unwanted frequencies. MEMS resonators with high $Q$, high resonant frequency, and low $R_{X}$ can be coupled either electrically or mechanically to form a channel-select filter that operates in the ISM band.

My work on MEMS filter design has focused on mechanically coupled resonators. Mechanical coupling offers certain advantages over electrical coupling in MEMS filters, including superior shape factor and stop band rejection. However, electrically coupled filters provide more dynamic control [10] and may be more suitable depending on the application.

Figure 2.1 illustrates the principle of mechanical coupling between resonators to form a filter. A simple two-pole filter can be formed by mechanically coupling two resonators. The can be modelled as two mass-spring-damper systems coupled together with a weak spring $K_{c}$. The eigenmodes of the tworesonator system occur when the resonators oscillate in phase and out of phase,

depicted in Figure 2.1. When the resonators are in phase, the coupling spring between them is never extended or contracted, and does not contribute to the res- 
onant frequency. The eigenfrequency of the mode is just the natural frequency of each resonator

$$
f_{1}=\frac{1}{2 \pi} \sqrt{\frac{K_{e f f}}{M_{e f f}}} .
$$

When the two resonators oscillate out of phase, the coupling spring between them is extended and contracted with maximum displacement, increasing the effective stiffness of each resonator. The eigenfrequency of this mode is

$$
f_{2} \approx f_{1}\left(1+\frac{K_{c}}{K_{e f f}}\right)
$$

with the assumption that $K_{c} \ll K_{e f f}$. This assumption also implies that the two resonant modes fall close together, forming a passband. The bandwidth of the resulting filter is given by

$$
\Delta f=f_{2}-f_{1} \approx f_{1} \frac{K_{c}}{K_{e f f}}
$$

This principle can be extended to multi-resonator systems, with multiple eigenmodes comprising the filter passband.

The desired filter characteristics are determined by the MEMS resonators comprising the filter. Low insertion loss is achieved by reducing $R_{X}$ while shape factor is defined by the resonators' quality factor. Previously, the high frequencies necessary to operate near the ISM band were achieved by reducing the air gap and increasing the transducer area of bulk-mode bar resonators [11]. Despite their high frequency, the large $R_{X}$ of these resonators would result in a high insertion loss filter. As discussed in $\$ 1.2 .2$ an attempt to reduce $R_{X}$ by filling the air gap of a wine-glass disk resonator with silicon nitride resulted in a resonant frequency of $165 \mathrm{MHz}$ with a $Q$ of 21,400 and an $R_{X}$ of $8.5 \mathrm{k} \Omega$ [6]. Alternatively, 


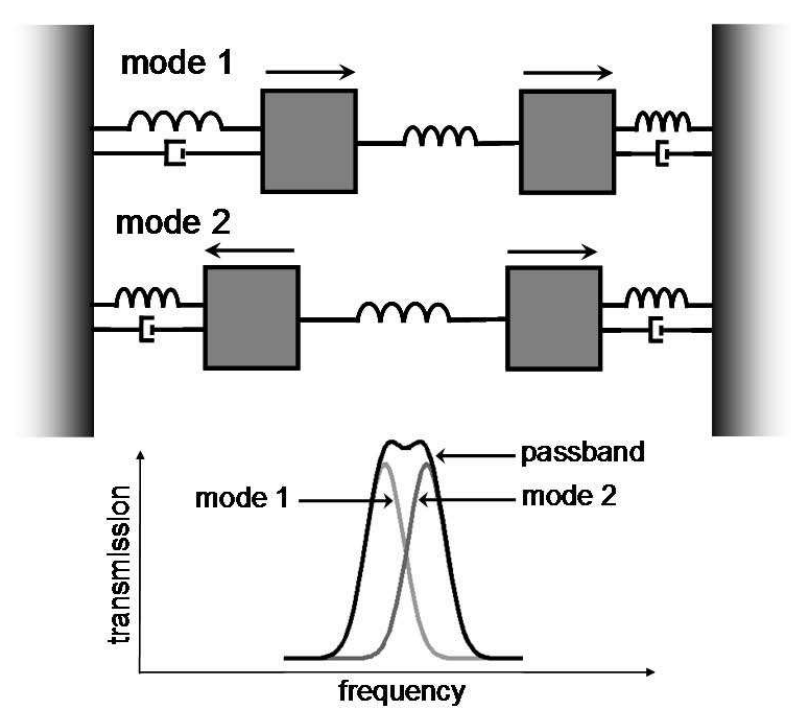

Figure 2.1: Two-pole mechanical filter, comprising two mass-springdamper systems joined by a coupling spring.

contour-mode aluminum nitride piezoelectric resonators with $656 \mathrm{MHz}$ resonant frequency and an $R_{X}$ of $170 \Omega$ have been reported. However, the ladder filter comprised of these low $Q$ piezoelectric resonators exhibited a $20 \mathrm{~dB}$ shape factor of $2.7[12]$.

\subsection{Thickness-Shear Mode Filters}

The thickness shear mode resonator serves as a good candidate for radio front end filter design. Its high- $Q$ mode has been proven successfully in quartz resonators and readily achieves high frequency and low motional impedance. The thickness shear mode resonator also exhibits a small range of frequency tuning capability which is useful for compensation of frequency shift due to fabrication errors. Dielectric transduction is achieved here by sandwiching a silicon nitride $(\kappa \sim 7)$ thin film between the silicon bar resonator and polysilicon electrodes, as 
shown in Figures 2.2 and 2.3. This enhances both the force density of the actuator as well as the sense capacitance, thereby improving the resonator's motional impedance by a factor of $\kappa^{2}$, as explained in $§ 1.2 .1$.

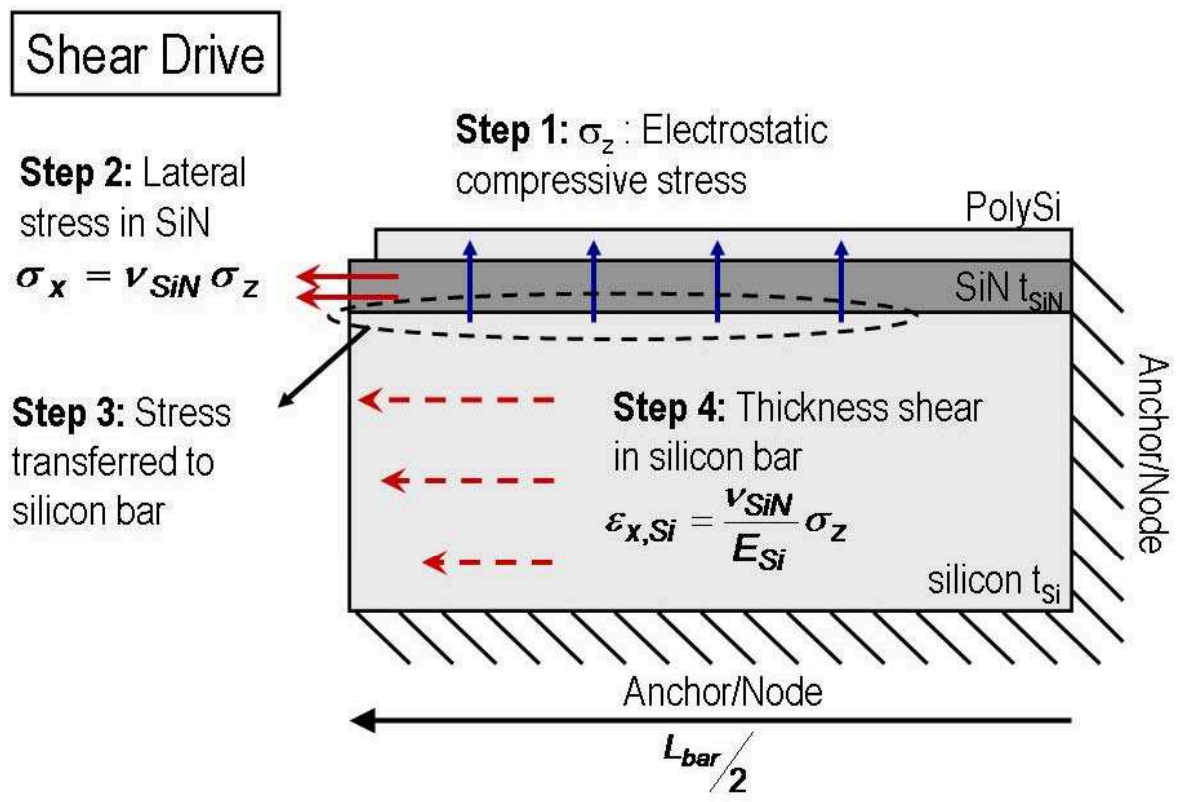

Figure 2.2: Driving mechanism for shear acoustic vibrations in a resonator using lateral dielectric transduction.

\subsubsection{Quarter-Wave Thickness Shear Bar Resonator}

The asymmetric thickness shear mode is common in quartz resonators, excited by the application of an AC signal across electrodes on opposing faces of a quartz crystal [13]. Unlike the quartz thickness shear mode, the electrode configuration of the dielectrically transduced silicon bar induces a symmetric mode, as shown in Figure 2.4. 


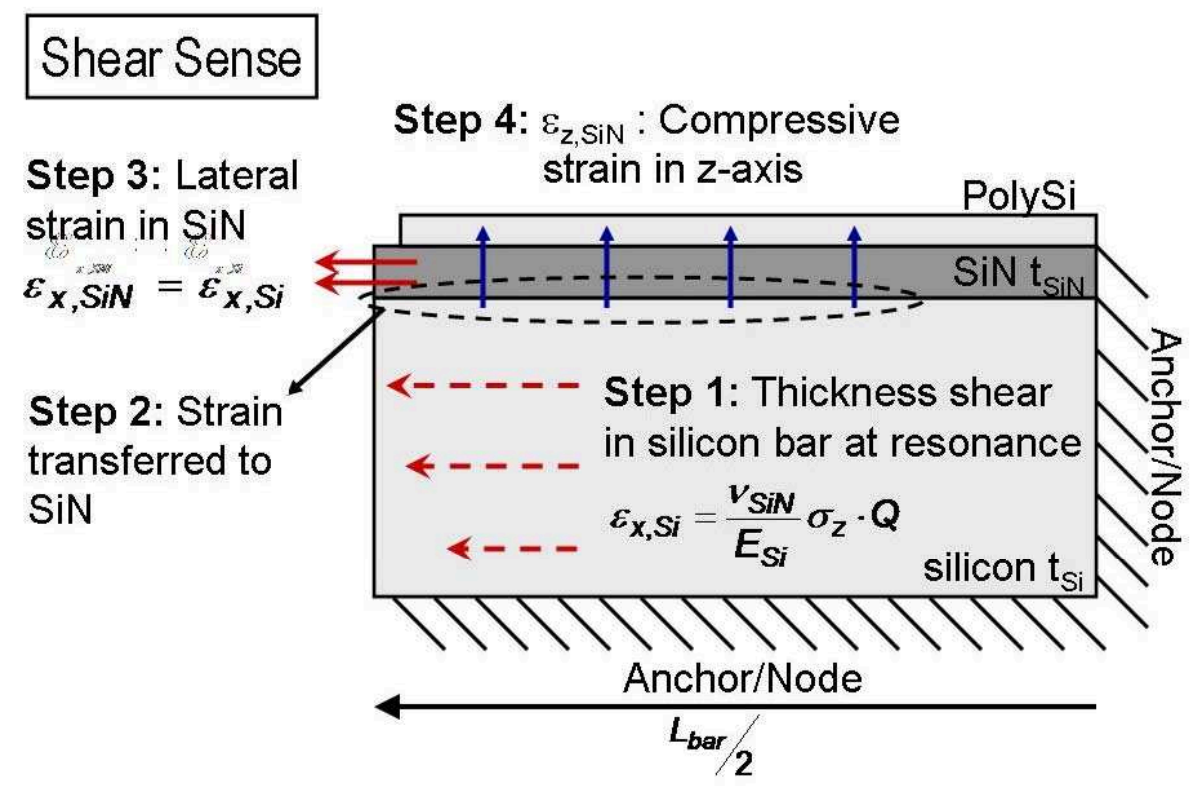

Figure 2.3: Sensing mechanism for shear acoustic vibrations in a resonator using lateral dielectric transduction.

A one-dimensional thickness shear mode is derived for an unreleased silicon bar transduced by a thin dielectric film. The film, deposited on top of the bar, is sandwiched between the silicon bar and conducting top electrodes (Figure 2.5). The bottom face of the bar is fixed to an effectively infinite oxide layer, imposing a zero-displacement boundary condition at the base of the bar. In this configuration, the silicon bar is biased to a DC voltage $V_{D C}$ while a small AC voltage $v_{i n}$ is applied to the top electrode. The voltage drop across the electrode-bar parallel plate capacitor induces a normal force on the dielectric film, transferring to a lateral strain in the dielectric. Though the strain is in fact uniform along both width and length of the beam, we consider a long narrow beam, such that a one dimensional approximation of the strain may be implemented. This strain is distributed between the film and silicon beam, inducing a thickness-shear reso- 

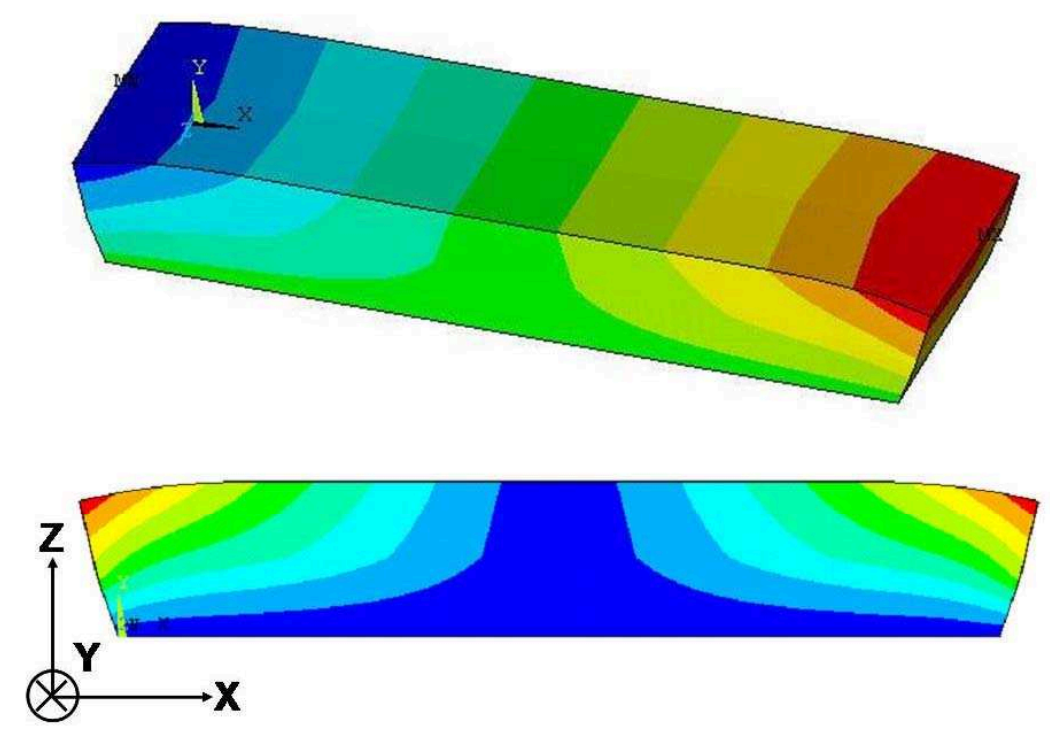

Figure 2.4: X-displacement contour plot from Ansys simulation of the symmetric quarter-wave thickness shear mode.

nance mode in the beam.

The lateral displacement $u_{x}$ for a one-dimensional thickness shear mode is given by

$$
u_{x}(x, z, t)=A x \sin \left(\omega \sqrt{\frac{\rho}{G_{S i}}} z\right) e^{i \omega t}
$$

imposing a resonant frequency of

$$
\omega=\frac{\pi}{2 b} \sqrt{\frac{G_{S i}}{\rho}} .
$$

A voltage $V_{D C}+v_{\text {in }}$ applied across the dielectric film generates a normal force

$$
\begin{aligned}
F_{z} & =-\frac{1}{2}\left(V_{D C}+v_{i n}\right)^{2} \frac{\partial C}{\partial z} \\
& \approx-V_{D C} v_{i n} \frac{\kappa_{f} \varepsilon_{0} w L}{2 g^{2}}
\end{aligned}
$$




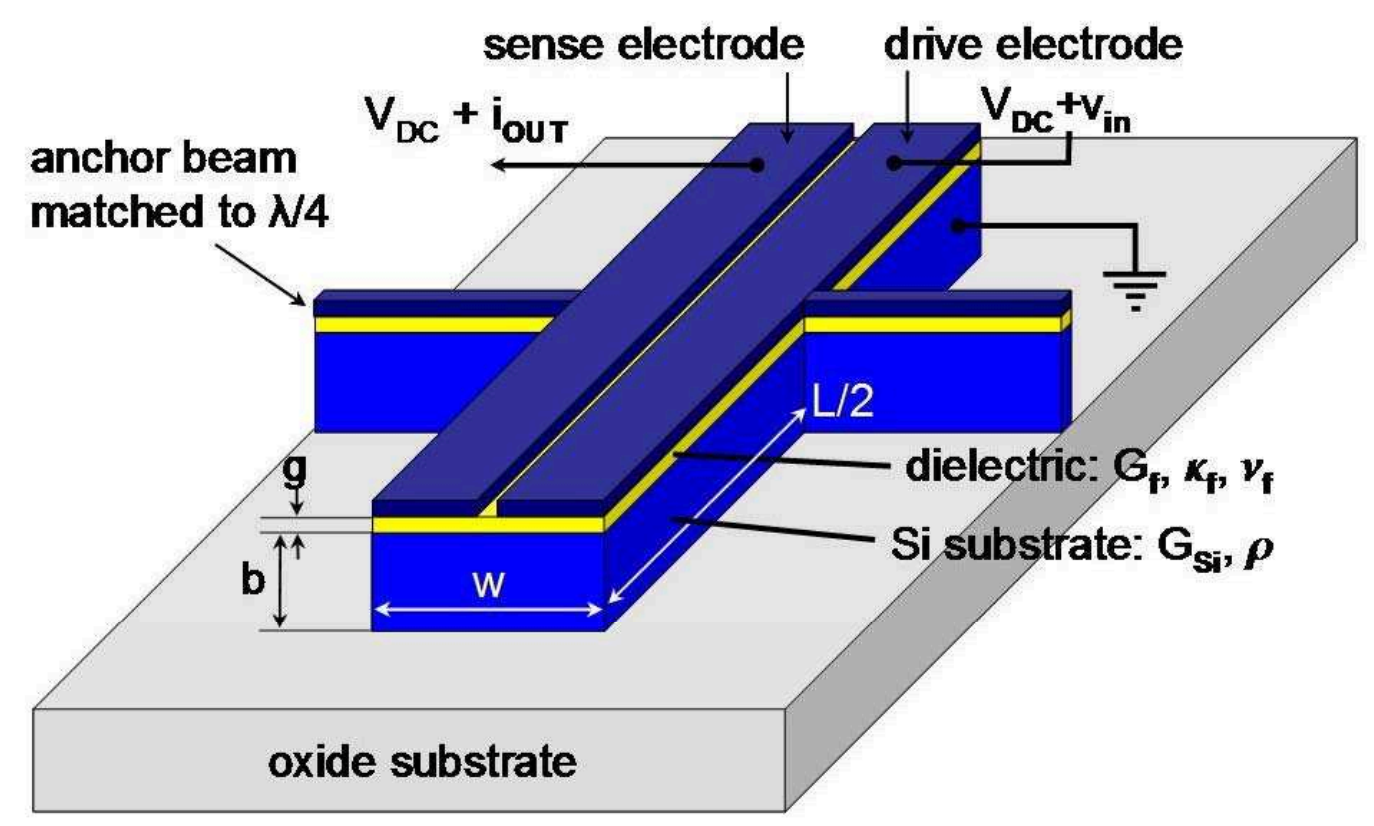

Figure 2.5: Unreleased thickness shear bar diagram.

for a beam of width $w$ and length $L$, where $\kappa_{f}$ and $g$ are the relative permittivity and thickness of the dielectric film, respectively. Here, we make the approximation that $v_{i n} \ll V_{D C}$. The factor of 2 in the denominator arises from a configuration in which the drive electrode covers half the width of the beam, and the sense electrode occupies the other half. The laterally transferred strain in the film is then

$$
\varepsilon_{y, f}=v_{f} \varepsilon_{z}=\frac{v_{f} F_{z}}{Y_{f} w L}
$$

so that the lateral stress in the film is then

$$
\sigma_{0}=Y_{f}^{*} \varepsilon_{y, f}=\frac{v_{f} F_{z}}{\left(1-v_{f}\right) w L}
$$


where $Y_{f}$ is the axial Young's modulus of the dielectric film. This initial lateral stress $\sigma_{0}$ functions like an effective residual stress in the film, consequently distributing itself in both film and bulk silicon. We assume that the shear stress in the silicon beam is distributed linearly through the beam. This yields a maximum shear stress in the beam of

$$
\begin{aligned}
\sigma_{0, b} & =\frac{G_{S i} g}{G_{f} g+\frac{1}{2} G_{S i} b} \sigma_{0} \\
& \approx \frac{2 g}{b} \sigma_{0}
\end{aligned}
$$

in the approximation that $g \ll b$. The maximum displacement of the Si bar can then be approximated as

$$
u_{x, \max }=\frac{b}{G_{S i}} \sigma_{0, b}=\frac{v_{f} K_{f} \varepsilon_{0} V_{D C} v_{i n}}{\left(1-v_{f}\right) G_{S i} g} .
$$

With the first-order determination of the quarter-wave thickness shear mode, one can calculate the motional impedance $R_{X}$, inductance $L_{X}$, and capacitance $C_{X}$ of the resonator. The change in the sensed capacitance over time is approximated as

$$
\frac{\partial C}{\partial t} \approx \frac{Q \omega K_{f} \varepsilon_{0} w L}{2 g^{2}} \Delta h_{\max }
$$

The quality factor $Q$ is introduced here to account for effective force amplification at resonance. $\Delta h_{\max }$ is the maximum change in the thickness of the dielectric film, and is given by $\Delta h_{\max }=v_{f} u_{x, \max }$. That is, the film is laterally expanded and contracted as the beam resonates, causing the thickness of the film to change 
with a Poisson efficiency factor. The output current is then

$$
i_{\text {out }}=V_{D C} \frac{\partial C}{\partial t}=\frac{Q\left(v_{f} \kappa_{f} \varepsilon_{0} V_{D C}\right)^{2} \pi w L}{4 g^{3} b\left(1-v_{f}\right) \sqrt{G_{S i} \rho}} v_{\text {in }} .
$$

The motional impedance of thickness shear resonator is

$$
R_{X}=\frac{v_{\text {in }}}{i_{\text {out }}}=\frac{4 g^{3} b\left(1-v_{f}\right) \sqrt{G_{S i} \rho}}{Q\left(v_{f} \kappa_{f} \varepsilon_{0} V_{D C}\right)^{2} \pi w L}
$$

The effective mass for the thickness shear mode is

$$
M_{e f f}=\frac{\rho w}{L^{2}} \int x^{2} \sin ^{2}\left(\omega \sqrt{\frac{\rho}{G_{S i}}} z\right) d x d z=\frac{1}{6} \rho w L b
$$

and the effective spring constant is

$$
K_{e f f}=M_{e f f} \omega^{2}=\frac{\pi^{2}}{24} \frac{G_{S i} w L}{b} .
$$

From Equations 2.13-2.15, for $R_{X} \equiv \sqrt{K_{e f f} M_{e f f}} / Q \eta^{2}$, the coupling constant $\eta$ is given as

$$
\eta^{2} \approx \frac{\left(\pi w L V_{D C} v_{f} \kappa_{f} \varepsilon_{0}\right)^{2}}{24\left(1-v_{f}\right) g^{3} b}
$$

The motional capacitance $C_{X} \equiv \eta^{2} / K_{e f f}$ is then

$$
C_{X}=\frac{\left(V_{D C} v_{f} \kappa_{f} \varepsilon_{0}\right)^{2} w L}{\left(1-v_{f}\right) g^{3} G_{S i}}
$$

and the motional inductance $L_{X} \equiv M_{e f f} / \eta^{2}$ is

$$
L_{X}=\frac{4 \rho b^{2} g^{3}\left(1-v_{f}\right)}{\left(V_{D C} v_{f} K_{f} \varepsilon_{0}\right)^{2} \pi^{2} w L}
$$




\subsubsection{Fabrication}

Figure 2.6 illustrates an SOI-based process for the fabrication of lateral dielectrically transduced resonators such as the thickness-shear bar resonator discussed here. In the case of these bar resonators, a $68 \mathrm{~nm}$ silicon nitride thin film was first deposited by LPCVD at $850^{\circ} \mathrm{C}$ on an n-type low resistivity SOI wafer with a $1.8 \mu \mathrm{m}$ thick SCS device layer. The silicon nitride was patterned to open contact holes to bias the silicon resonator. A $120 \mathrm{~nm}$ layer of n-doped polysilicon was then deposited by LPCVD at $620^{\circ} \mathrm{C}$, annealed at $1000^{\circ} \mathrm{C}$ for 40 minutes, and patterned to form the electrodes. This was followed by a reactive ion etch RIE step to define the resonator into the silicon device layer.

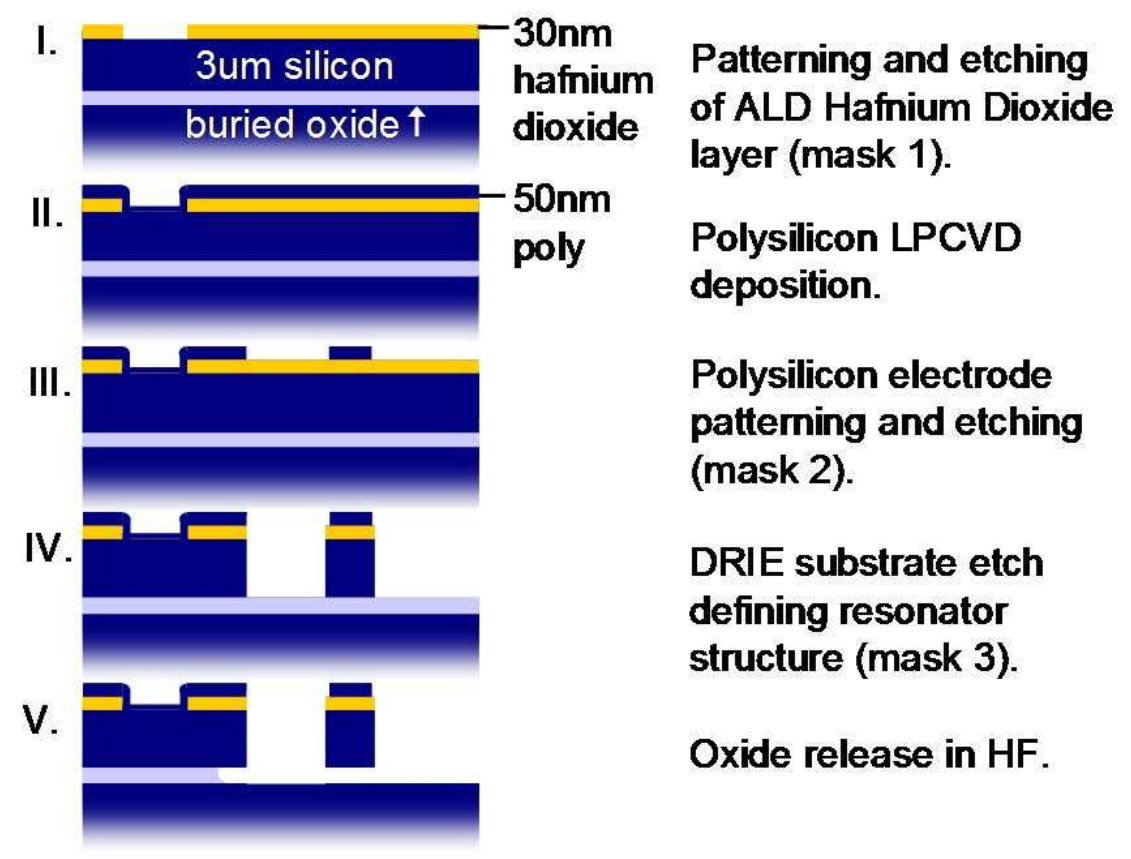

Figure 2.6: SOI-based micro-fabrication process for lateral dielectrically transduced resonators. 


\subsubsection{Experimental Results}

An unreleased $80 \mu m \times 40 \mu m$ wide bar resonator was characterized using a DesertCryo microwave probe station. The resonator body was grounded and a DC bias was applied to both the drive and sense electrodes with bias-Ts from MiniCircuits. Transmission measurements were performed using an Agilent 8753ES Network Analyzer and the quality factor and insertion loss were extracted from the measured data. The motional impedance of the resonator was determined from the insertion loss data after adjusting for the attenuation losses at the drive pad. The quarter-wave thickness shear vibration mode of the unreleased silicon resonator was measured with a resonant frequency of $713 \mathrm{MHz}$, an $R_{X}$ of 10.5 $k \Omega$ and $Q$ of 1517 in air (Figure 2.7).

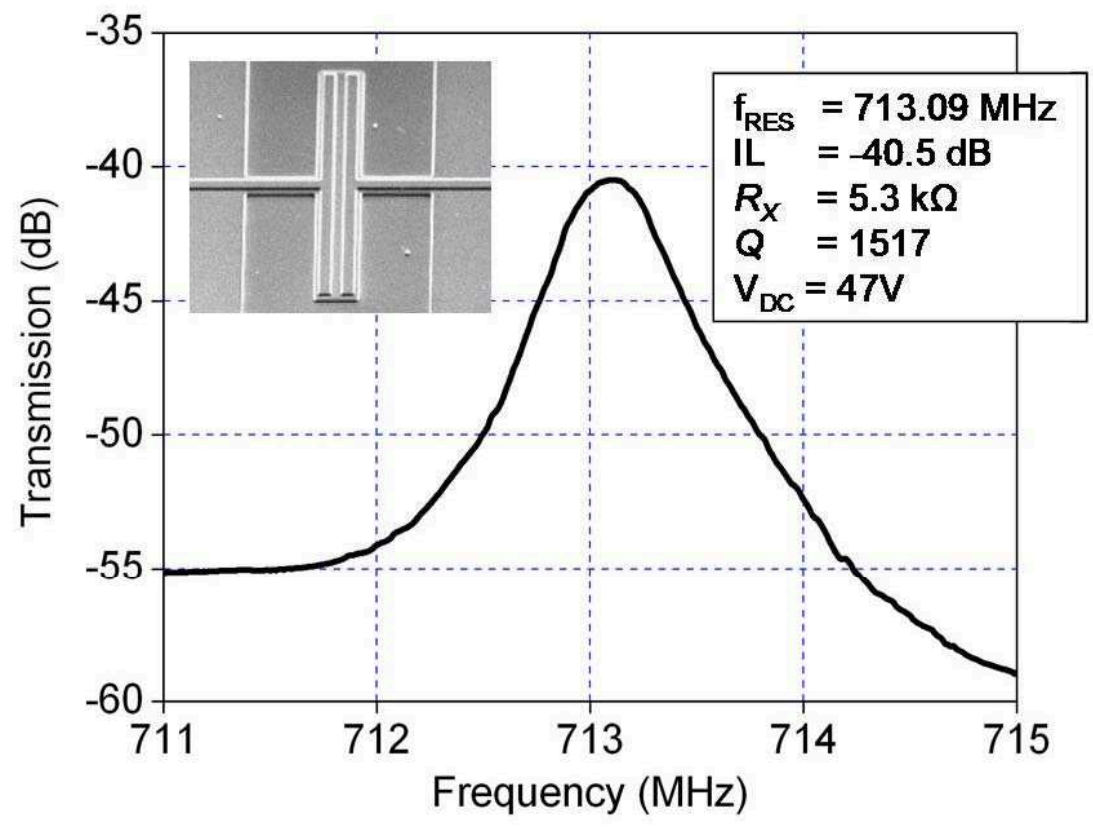

Figure 2.7: SEM of a silicon nitride-on-silicon unreleased bar resonator. Measured transmission of the thickness shear mode of the unreleased resonator in air. 
It has previously been shown that quality factor improves with reduced anchor area. By performing a timed etch of the buried oxide in HF, the overall contact area between the oxide and bottom surface of the resonator was reduced to approximately $30 \mu m \times 5 \mu m$. This partially released bar had a resonant frequency of $723 \mathrm{MHz}, R_{X}$ of $2.4 k \Omega$ and $Q$ of 4,400 in air (Figure ??). The large acoustic mismatch of the air gap reduces leakage of the shear standing wave into the anchor, thereby decreasing the quarter wavelength of the vibration mode. This explains the increased resonant frequency of the partially released bar relative to the unreleased bar.

The frequency response of the partially released bar gives a coupling factor $k_{e m}^{2} \equiv f_{\text {zero }} / f_{\text {pole }}-1 \approx C_{X} / C_{F T}$ of $k_{e m}=0.03$. This is comparable to the coupling factor of quartz crystals [14].

Previous attempts at frequency tuning forced a change in the effective stiffness of the resonator through deformations in the direction of resonant motion. However, the stiffness of high frequency resonators is very large in this direction, requiring considerable forces to tune the device. For example, a $1 \mathrm{GHz}$ resonator has a stiffness on the order of $1 \mathrm{MN} / \mathrm{m}$ in the resonant direction. In the case of the partially released thickness shear bar resonator, the stiffness orthogonal to the resonant motion (z-direction) is very low. A static flexure of the bar as shown in Figure 2.9(left) reduces the effective shear stiffness. Varying the tuning voltage from 50 to $150 \mathrm{~V}$ results in a $5 \mathrm{MHz}$ change in resonant frequency with negligible degradation in quality factor (Figure 2.9(right)).

The high tuning voltages can be reduced significantly by decreasing the buried oxide thickness. Furthermore, scaling to higher frequencies will increase the tuning range, as the bar stiffness in the direction of tuning is proportional to $b^{3}$. This frequency tuning capability demonstrated by the thickness shear bar is 


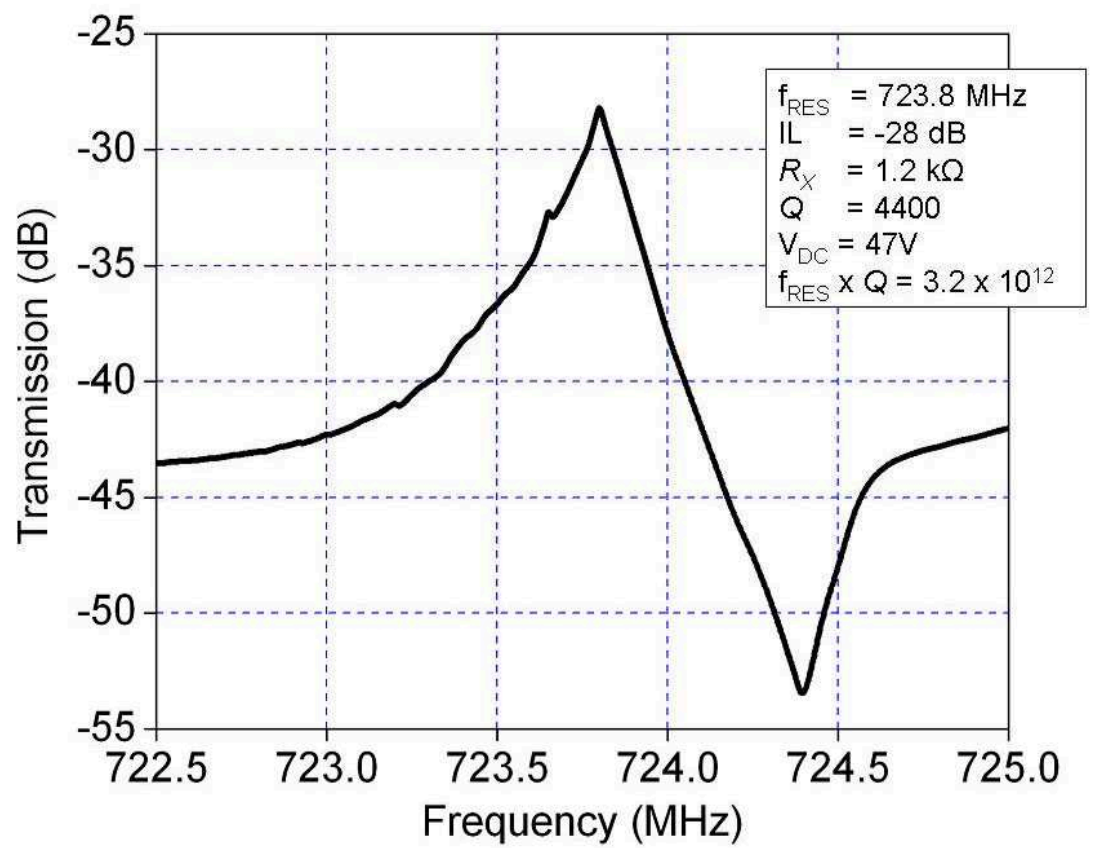

Figure 2.8: Measured transmission of the shear mode of the partially released resonator in air. The reduction in anchor size increased the quality factor almost three-fold.

highly beneficial for frequency shift compensation due to fabrication errors.

Thickness shear mode resonators using dielectric transduction achieve high frequencies with greatly improved motional impedance. The lateral electrode design causes a Poisson ratio inefficiency in the transfer of vertical strain into lateral strain. However, this transfer is necessary to induce a shear resonance. Furthermore, this configuration allows for a large electrode area, significantly decreasing the resonator's motional impedance. 

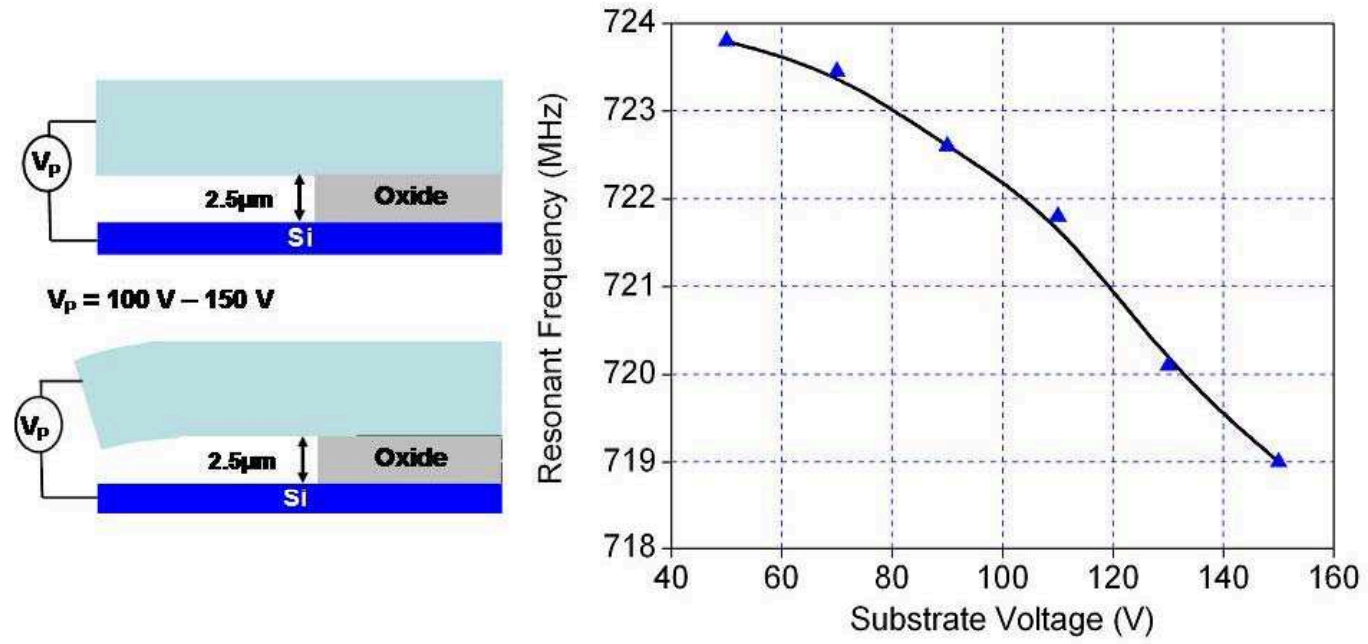

Figure 2.9: (left) Tuning schematic for the partially released bar resonator, applying a bias voltage to bend the resonator perpendicular to the resonant direction of motion. (right) Resonant frequency vs. tuning voltage $V_{P}$ of the bar resonator. A tuning range of about $5 \mathrm{MHz}$ is observed.

\subsubsection{Half-Wave Thickness Shear Bar Filters}

The lateral dielectric transduction implemented for quarter-wave thicknessshear mode resonators can be extended to the half-wave mode, obtained in fully-released bar resonators. To induce the half-wave mode, the buried oxide layer is fully etched, leaving a free displacement boundary condition on the bottom face of the bar.

Pure thickness-shear mode resonance of a bar depends only on thickness $b$, 
with frequency

$$
f=\frac{1}{2 b} \sqrt{\frac{G}{\rho}}
$$

where $G$ and $\rho$ are the shear modulus and mass density of the silicon resonator, respectively. In reality, the resonator exhibits a small-amplitude flexure mode coupled to the shear mode. This coupling can be observed in the ANSYS modal analysis in Figure 2.10. The Southwell-Dunkerley formula [15] approximates the combined shear-flexure frequency as

$$
\frac{1}{f_{\text {total }}^{2}}=\frac{1}{f_{\text {shear }}^{2}}+\frac{1}{f_{\text {flexure }}^{2}}
$$

Therefore, the silicon bar's lateral dimensions affect the resonant frequency, giving layout design flexibility covering a $30 \mathrm{MHz}$ range below $840 \mathrm{MHz}$. Figure 2.11 shows the bar's simulated resonant frequency as a function of the bar length. This property is exploited to fabricate multiple frequency resonators and filters on the same chip.

The half-wave thickness shear resonators were fabricated in an SOI process similar to Figure 2.6. The dielectric transducer was a $30 \mathrm{~nm}$ hafnium dioxide film $\left(\kappa \sim 28, v_{\text {acoustic }} \sim 8,500 \mathrm{~m} / \mathrm{s}\right)$ on a low resistivity SOI wafer with a $3.2 \mu \mathrm{m}$ thick SCS device layer. An SEM of the resonator is shown in Figure 2.12. The half-wave thickness shear mode of the released silicon resonator was measured with a resonant frequency of $809 \mathrm{MHz}$, a $Q$ of 7,800 and an $R_{X}$ of $59 \Omega$ in air (Figure 2.13).

A mechanically coupled thickness shear arc filter based on the principles described in $\S 2.1$ is shown in Figure 2.14. The three arc array is implemented instead of one wide shear arc to suppress plate modes in the resonators. Two arc arrays are mechanically coupled with a low velocity torsion beam. The arc filter 


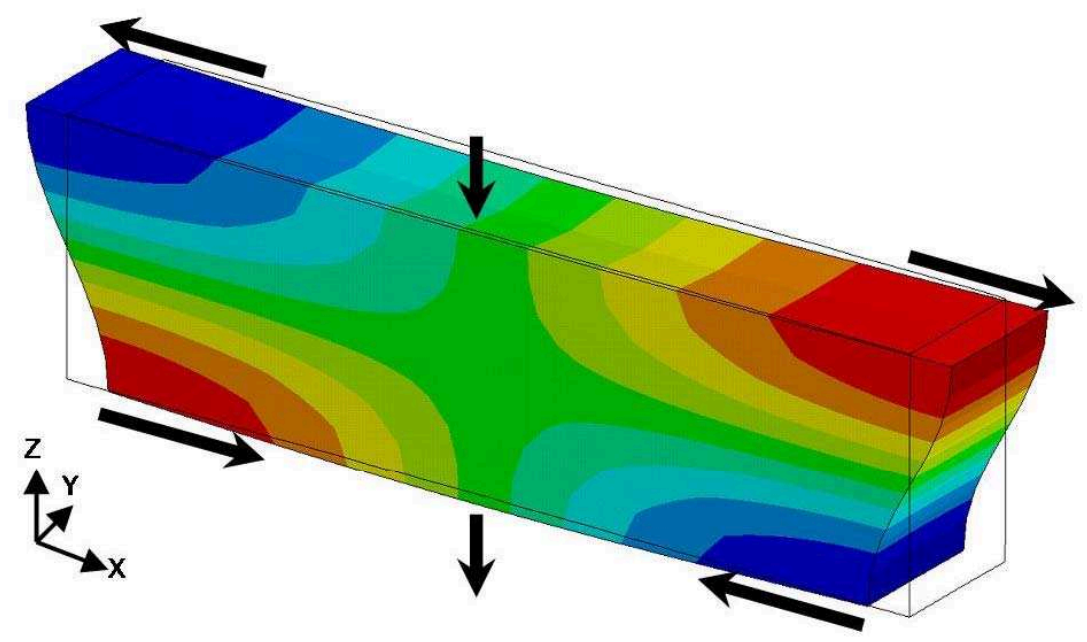

Figure 2.10: ANSYS contour plot of the symmetric half-wave thickness shear mode. This mode causes the tether suspension at the nodal plane to move along the Z-axis, as indicated by the down arrows.

symmetry accesses the full range of the coupling beam's effective stiffness, allowing for easy control of filter bandwidth at the expense of pass-band ripple. In order to access the low velocity coupling point, the three-arc-array filter is coupled near the nodal plane of the resonators. Figure 2.15 presents the frequency response and characteristics of the mechanically coupled filter measured in air with a $5 \mathrm{~V}$ bias and a termination impedance of $2 \mathrm{k} \Omega$.

\subsection{Balanced-Unbalanced MEMS Filter}

Single-ended to differential micromechanical filters with large stop band rejection are ideal replacements for conventional SAW and FBAR filters [16, 17] in 


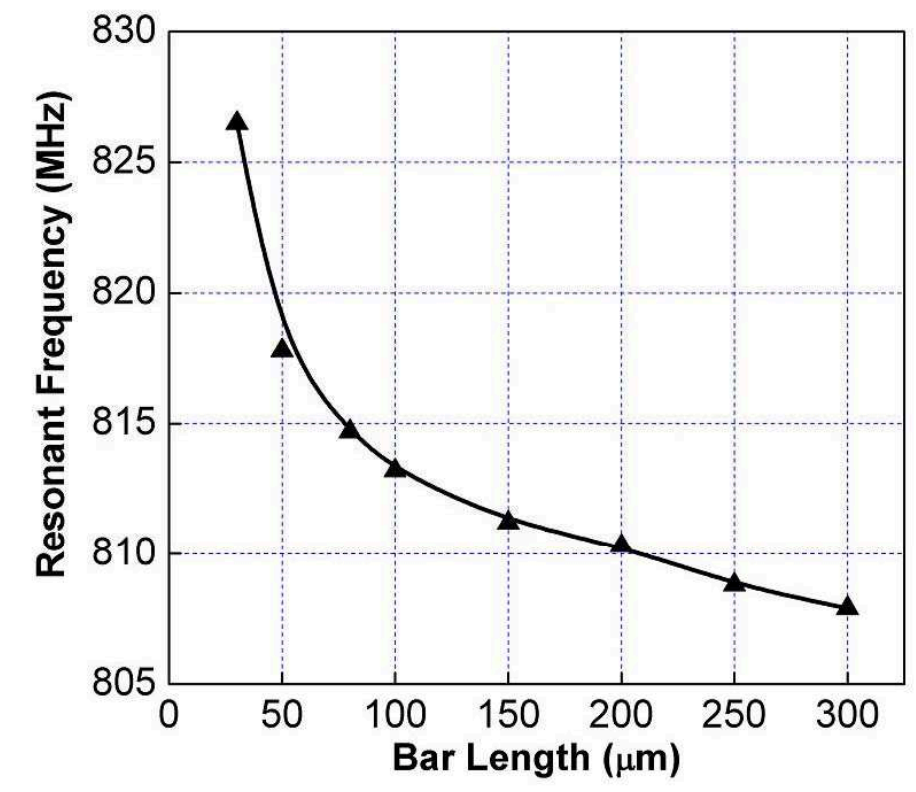

Figure 2.11: ANSYS simulation of length $(L)$ vs. resonant frequency of the $3.2 \mu \mathrm{m}$ thick half-wave shear mode resonator. The pure shear mode resonant frequency of the bar is $844 \mathrm{MHz}$.

sensor network transceivers and GSM and W-CDMA cell phones, which depend on differential signal paths. A differential output from the front-end filter eliminates the need for an off-chip balanced-unbalanced transformer (balun) in front-end radio design and increases filter linearity (Figure 2.16). The design and performance of a single-ended input to differential output resonant electromechanical filter are presented, yielding a $425 \mathrm{MHz}$ center frequency with 1 $\mathrm{MHz}$ bandwidth (BW), $8 \mathrm{~dB}$ insertion loss (IL), $<5 \mathrm{~dB}$ pass-band ripple, $-50 \mathrm{~dB}$ stop-band rejection, and $-48 \mathrm{~dB}$ common mode suppression (CMS), for a footprint of about $150 \times 150 \mu \mathrm{m}^{2}$.

Fully differential mechanical filters can be operated in single-ended to differential mode by providing only one of two input signals to actuate the filter. However, this method is not optimal since half of the drive electrode area is not utilized, resulting in a higher-than-necessary input motional impedance 


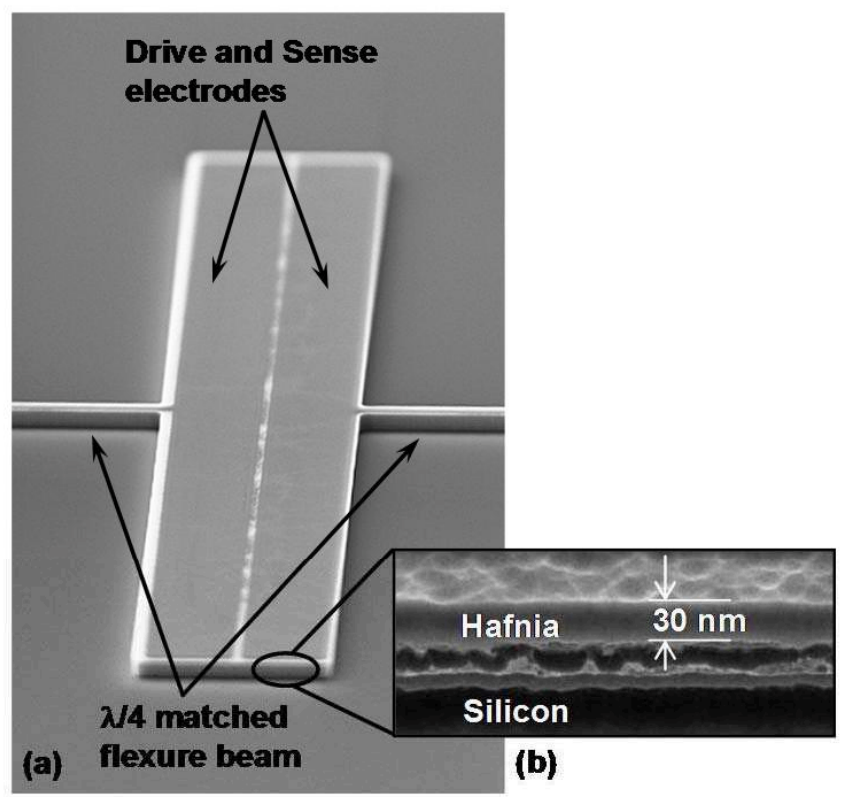

Figure 2.12: (a) Scanning electron micrograph of a hafnium dioxide-onsilicon fully released bar resonator. (b) Scanning electron micrograph of the $30 \mathrm{~nm}$ hafnium dioxide layer on the top of the silicon resonator.

$\left(R_{X}\right)$. The design presented here couples an intrinsically single-ended driving resonant mode to an inherently differential sensing resonant mode, thereby optimizing electrode area. The mechanical coupling between two different types of resonators enables us to achieve a CMS of $-48 \mathrm{~dB}$, which is $20 \mathrm{~dB}$ better than previously demonstrated bulk acoustic wave single-ended to differential filters $[18,19]$.

Lateral high- $\kappa$ dielectric transduction increases both actuation force density and sensing capacitance, providing a $\kappa^{2}$ reduction in the motional impedance of the resonators over similar air-gap transduced devices. These low $R_{X}$ constituent resonators enable the design of low insertion loss filters. Furthermore, the resonators' high motional inductance $\left(L_{X}\right)$, low motional capacitance $\left(C_{X}\right)$, and high quality factor $(Q)$ contribute to a small shape factor, making this fil- 


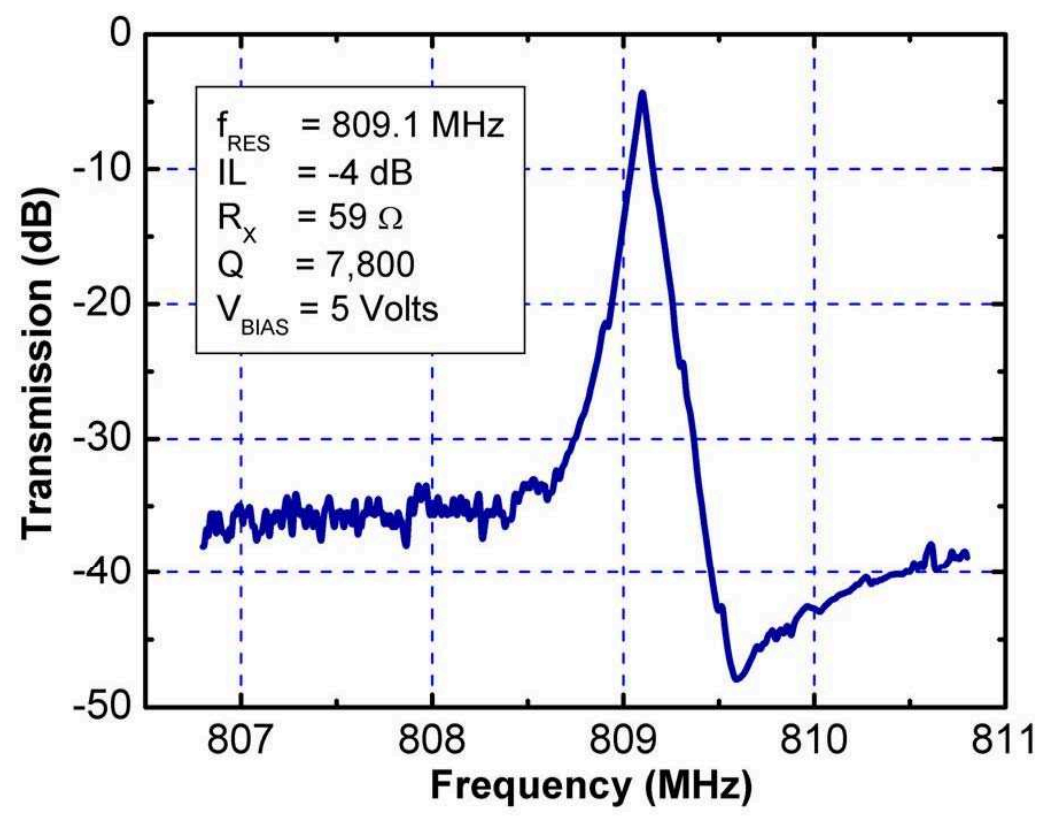

Figure 2.13: Measured transmission response of a half-wave thickness shear mode resonator in air. The $f . Q$ product of the resonator is $6.2 \times 10^{12}$.

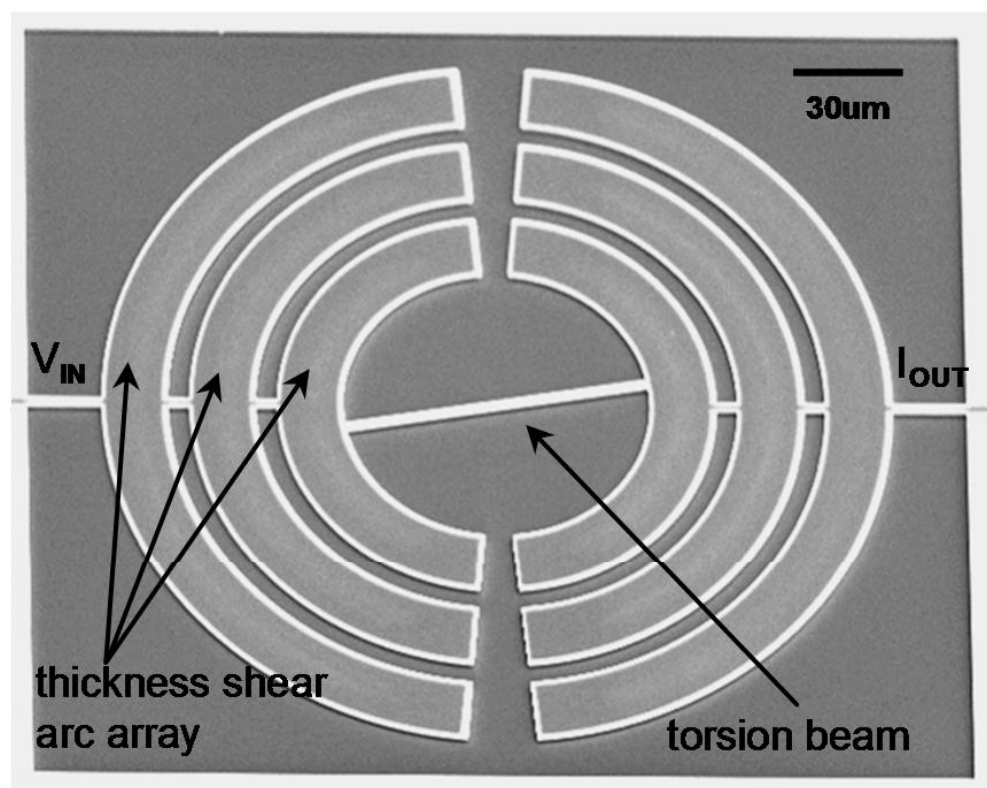

Figure 2.14: Scanning electron micrograph of a mechanically coupled shear arc filter. 


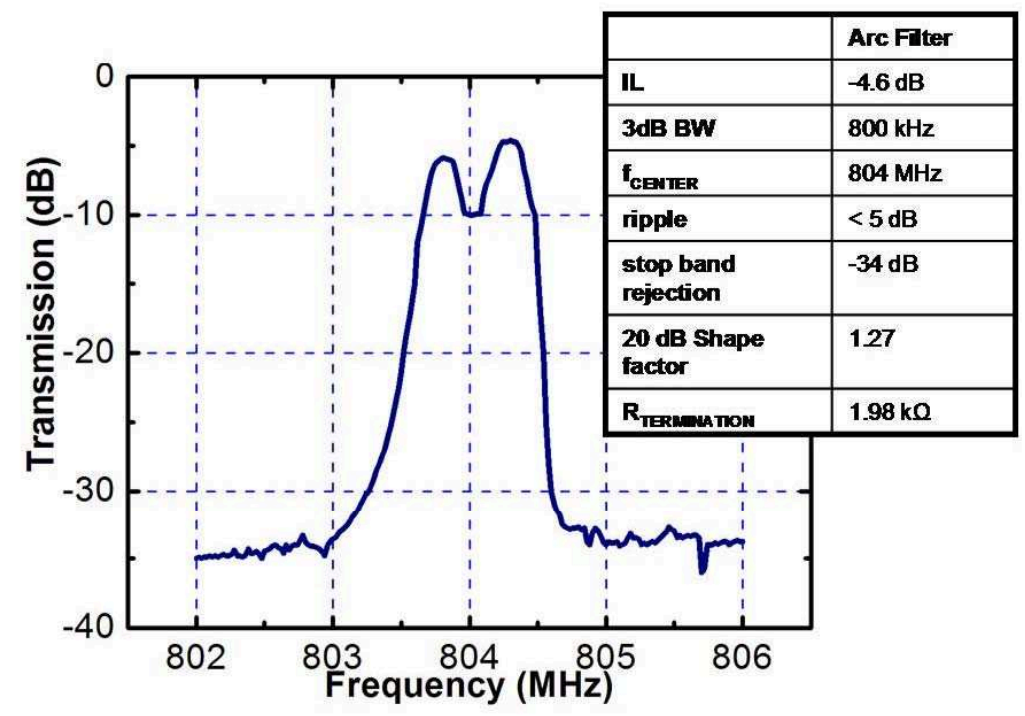

Figure 2.15: Transmission response of the mechanically coupled arc filter. The arc filter characteristics show improved stop-band rejection and $20 \mathrm{~dB}$ shape factor compared to the electrically coupled filters.

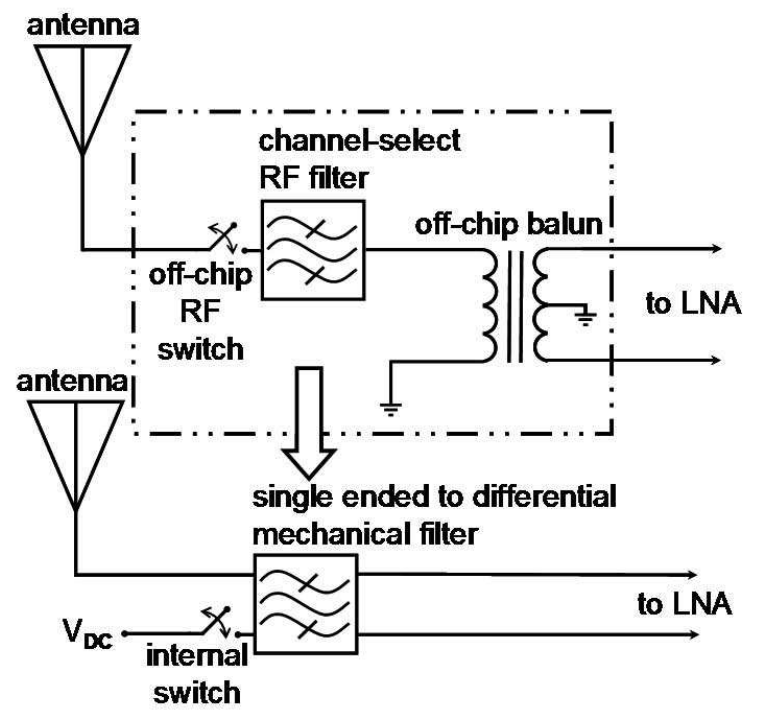

Figure 2.16: Schematic of a radio front end with in-line RF switch, filter, and balun transformer. These three components can be replaced with a single-ended to differential MEMS filter. 
ter ideal for channel-select narrow-band applications. Additionally, the dependence of resonant frequency on lateral dimensions allows for the fabrication of a switchable filter array covering a wide range of frequencies on a single chip. Each filter in the array can be switched on or off with the selective application of a DC polarization voltage to the device layer [20]. Lateral dielectric transduction for contour mode resonators is similar in principle to that of thickness-shear resonators discussed in this chapter, and is outlined in Figures 2.17 and 2.18.

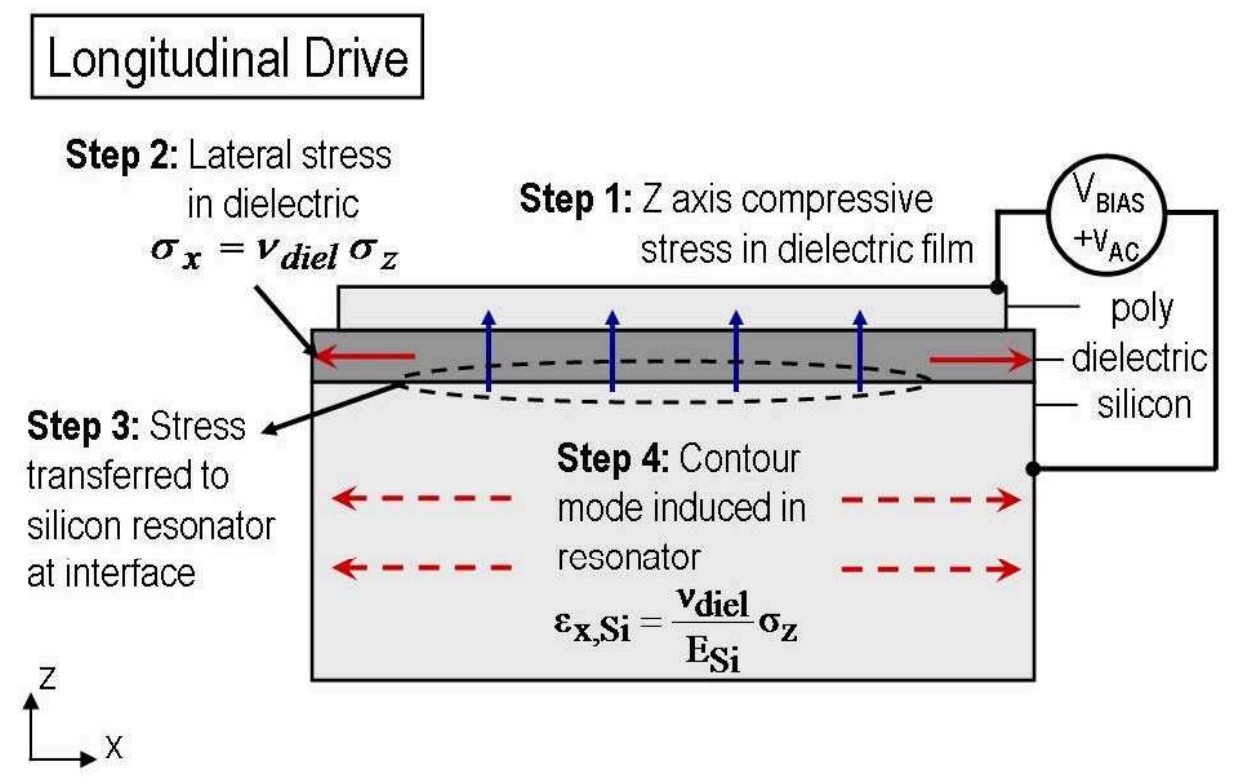

Figure 2.17: Driving mechanism for longitudinal acoustic vibrations in a resonator using lateral dielectric transduction.

The filter, fabricated in the process described in $\S 2.2 .4$, is comprised of two concentric resonators. An inner disk resonator $\left(R_{\text {disk }}=6.25 \mu \mathrm{m}\right)$ is dielectrically actuated to induce a radial contour mode [2]. Mechanical coupling from this inner disk to a concentric ring resonator drives a contour wine-glass mode in the outer ring $\left(R_{\text {in }}=32.6 \mu \mathrm{m}, R_{\text {out }}=42.3 \mu \mathrm{m}\right)$ [21]. The coupling springs' stiffness is re- 


\section{Longitudinal Sense}

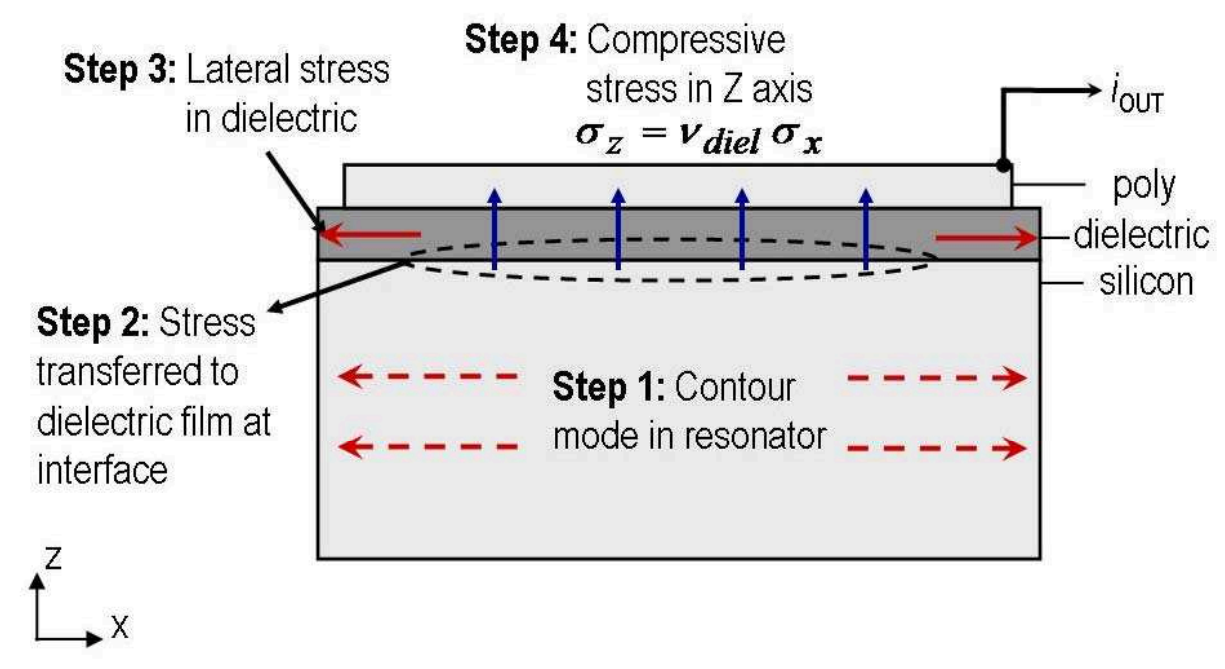

Figure 2.18: Sensing mechanism for longitudinal acoustic vibrations in a resonator using lateral dielectric transduction.

duced by ensuring a flexure mode coupling using perpendicular arc segments (Figure 2.19). The filter is suspended from the quasi-nodes of the wine glass ring. The anchor beams are frequency-matched to reflect back energy of the wine glass resonant mode but act as an energy sink for undesired ring modes.

The AC equivalent circuit of an electromechanical resonator is given by a series RLC circuit, where for a coupling constant $\eta=V \frac{\partial C}{\partial x}, R_{X}=\frac{b}{\eta^{2}}, C_{X}=\frac{\eta^{2}}{K_{e f f}}$, and $L_{X}=\frac{M_{e f f}}{\eta^{2}}$. The second-order electromechanical filter is modelled electrically by two resonator RLC circuits coupled through a capacitive $\mathrm{T}$ as illustrated in Figure 2.20. When the resonators oscillate in phase, the current flowing through the shunt capacitor $C_{C P}$ is minimized. When the resonators are out of phase, the current though the coupling shunt capacitor is at its maximum. In the mechanical domain, this corresponds to a minimal relative displacement of the coupling 


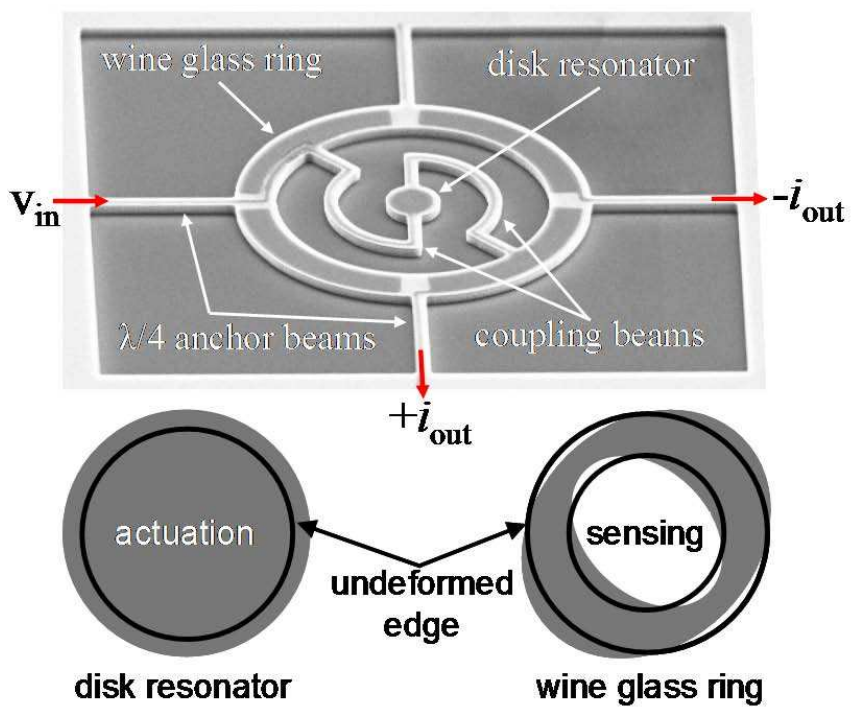

Figure 2.19: Scanning electron micrograph of single-ended to differential MEMS filter. The antenna signal drives resonance in a breathing-mode disk, which couples mechanically to a wine glass ring resonator.

beam for in-phase resonance and a maximum beam displacement for out-ofphase resonance. In this model, feed through capacitance is negligible due to the large distance between drive and sense electrodes.

The filter was tested on Cascade Microtech probe station, using an Agilent 8753ES Network Analyzer, a Minicircuits bias-T and 0/180 deg phase combiner. Figure 2.21 shows the $1 \mathrm{MHz}$ BW filter transmission response for a bias voltage of $5 \mathrm{~V}$ and termination impedance of $4.8 \mathrm{k} \Omega$. An insertion loss of $8 \mathrm{~dB}$, shape factor of 1.16 , stop band rejection of $-50 \mathrm{~dB}$, and $-48 \mathrm{~dB}$ common mode suppression are observed. The CMS improved by $20 \mathrm{~dB}$ over electrically coupled filters [18] due to the differential mechanical nature of the wine glass ring eigenmode. The filter has large insertion loss due to mismatch in the motional impedances of the constituent resonators. This can be improved by implementing a larger breathing mode ring for the inner resonator, increasing electrode area while maintain- 


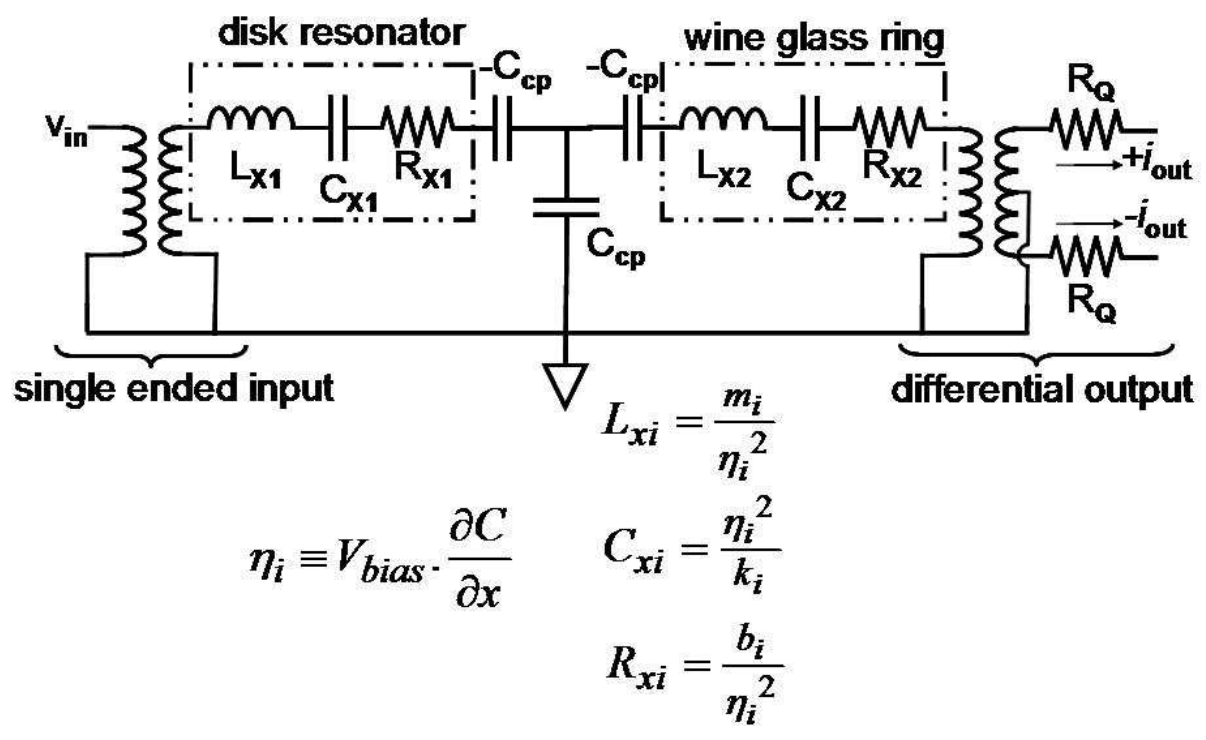

Figure 2.20: Equivalent circuit for single-ended to differential MEMS filter comprised of two types resonators coupled with a mechanical spring.

ing the necessary symmetry.

This single-ended to differential filter prototype offers a channel-select switchable low-power electromechanical alternative for front-end RF receivers, eliminating the need for off-chip baluns and external RF switches. The high inductance and low capacitance of the dielectrically transduced resonators comprising the filter provide a small shape factor for narrow BW applications. Mechanical coupling greatly improves stop-band rejection, while the inherently differential mode shape of the wine glass ring provides excellent common mode suppression. 


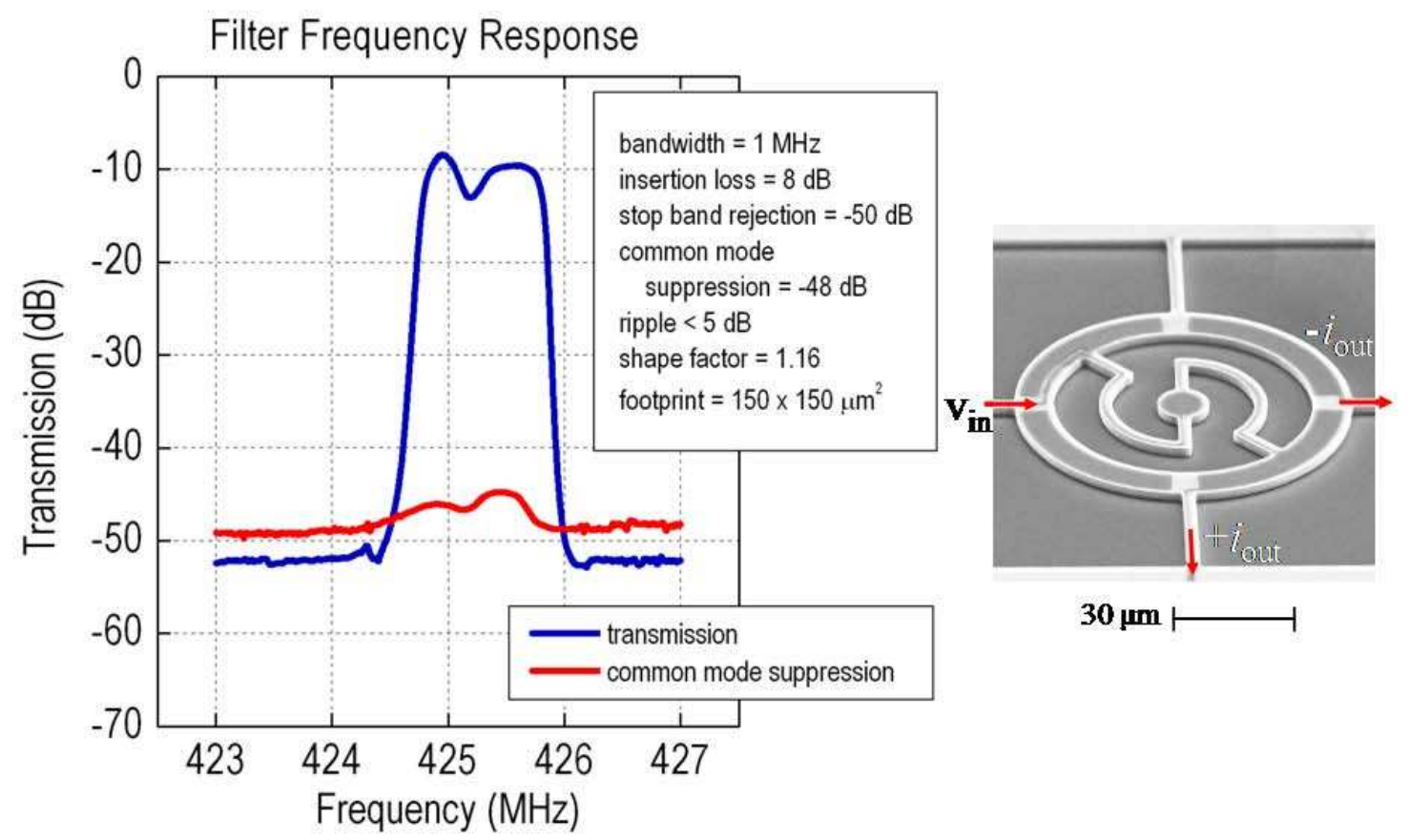

Figure 2.21: Measured frequency response of a $425 \mathrm{MHz}$ single-ended to differential filter.

\subsection{D Resonator Array For MEMS Filters}

\subsubsection{Theory}

Much of current MEMS resonator research focuses on channel-select filters for radio front-ends. One-dimensional and bridged arrays of MEMS resonators have been studied extensively to form narrow bandwidth filters at low $\mathrm{MHz}$ frequencies. To improve insertion loss, multiple resonators have been strongly coupled to increase transduction area in two-pole filters [22]. Alternatively, sets 


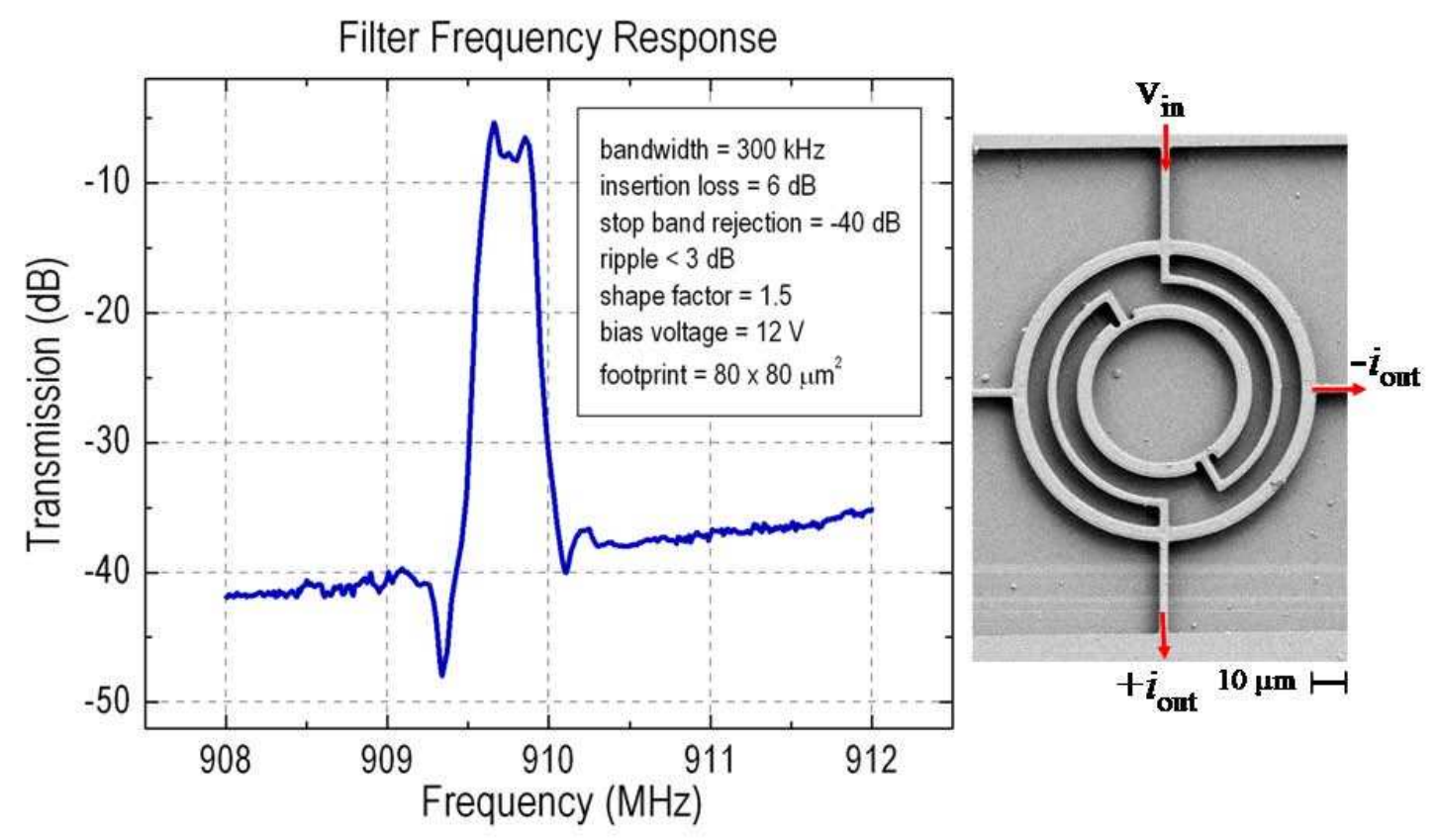

Figure 2.22: Measured frequency response of a $910 \mathrm{MHz}$ single-ended to differential filter.

of 1D filters coupled electrically in parallel have been proposed and demonstrated [23]. However, pure electrical coupling does not ensure a coherent summation of the filter passband due to small variations between each electrically parallel 1D filter. Some strong mechanical coupling is therefore required to drive an array of 1D filters coherently.

Though two-pole filters currently dominate RF MEMS filter research, there is an impetus to extend to multi-pole filters. As shown by Wang et al [24], increasing the number of resonators in a 1D filter improves both pass-band shape factor and stop-band rejection. However, spatial decay in the resonators and fabrica- 


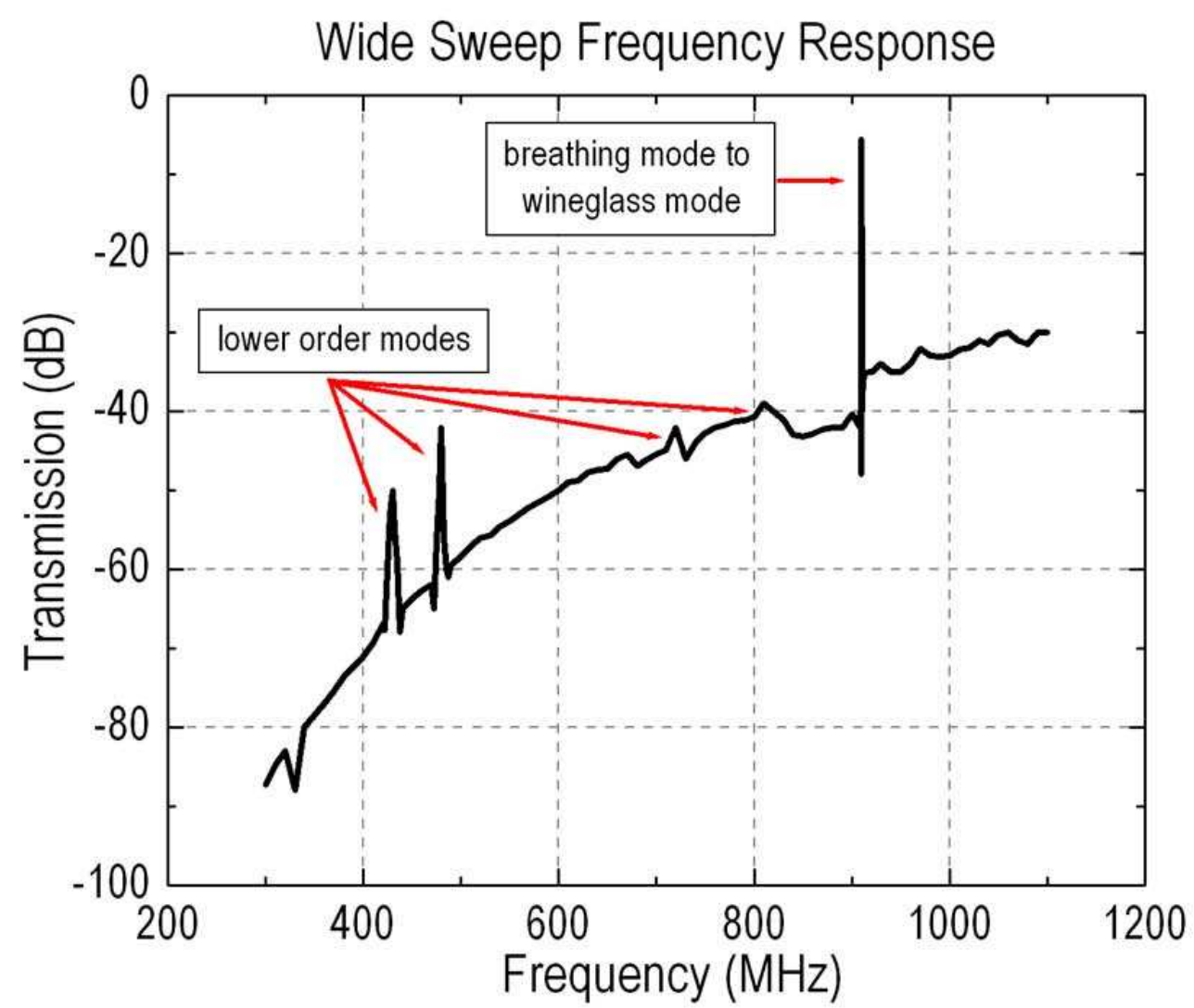

Figure 2.23: Broad frequency sweep of the $910 \mathrm{MHz}$ filter, showing lower frequency spurious modes suppressed below $-40 \mathrm{~dB}$.

tion variations result in increased insertion loss and distortion in the passband as more resonators are added to the $1 \mathrm{D}$ array. This phenomenon has previously been investigated by Castanier and Pierre [25], using classical perturbation theory to model the effects of both dissipation and variations on 1D filters.

To improve this passband distortion, Judge et al [26] proposed a 2D coupling which averages out the stochastic resonator characteristics. The design strongly couples an array of identical 1D filters, generating a two-dimensional matrix of resonators which are coupled weakly in one direction and strongly in the other (Figure 2.25). The weak coupling $\left(k_{C}\right)$ defines the filter passband, 

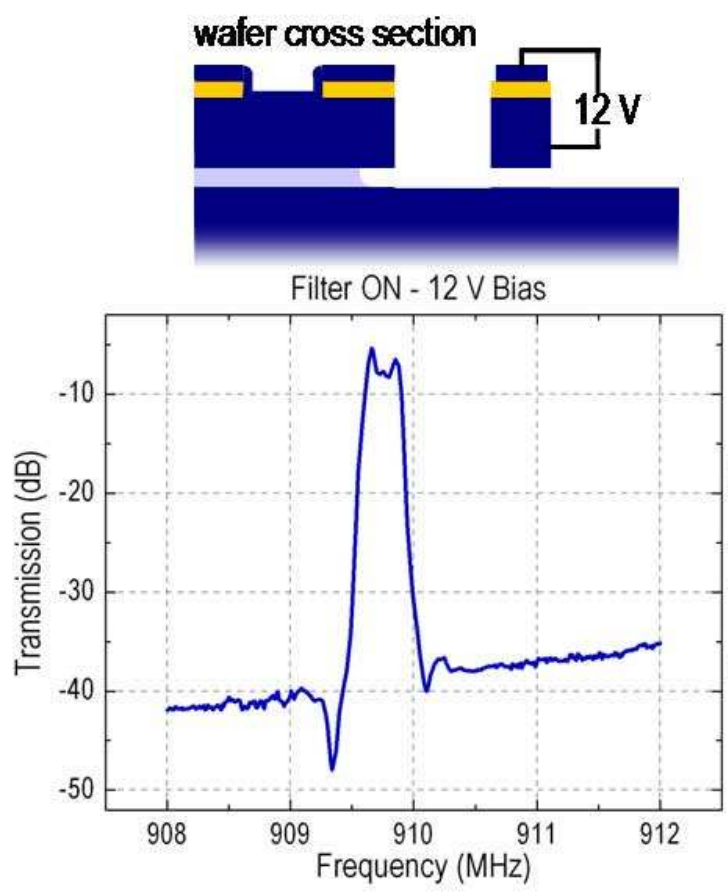

wafer cross section
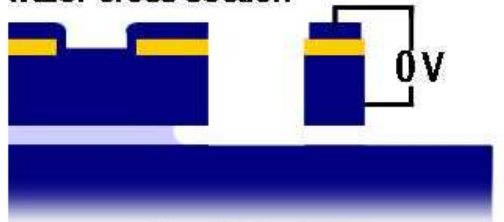

Filter OFF - O V Bias

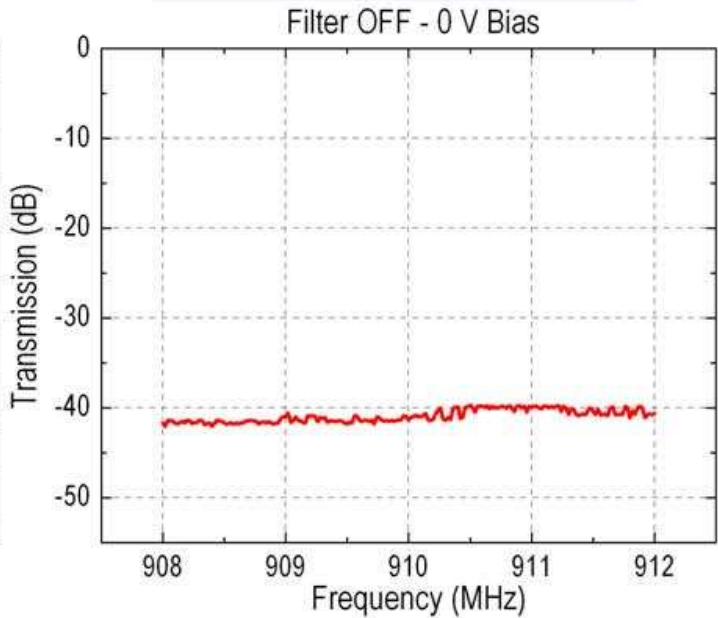

Figure 2.24: Switching capabilities of the MEMS filter. Applying a bias voltage across the dielectric transducer passes signals in the targeted passband. Switching that bias voltage off allows no signal to pass.

as in the case of the 1D filter. The number of resonators in the weak coupling direction therefore defines the number of poles in the passband. The strong coupling $\left(k_{S}\right)$ averages variations in the resonators due to fabrication tolerances. If $k_{C} \ll k_{S}$, and $N_{S}$ the number of resonators in the strong coupling direction, then the effective standard deviation of variations in the resonators is

$$
\sigma_{e f f}=\frac{\sigma}{\sqrt{\frac{N_{S}}{2}}}, N_{S} \text { even }
$$




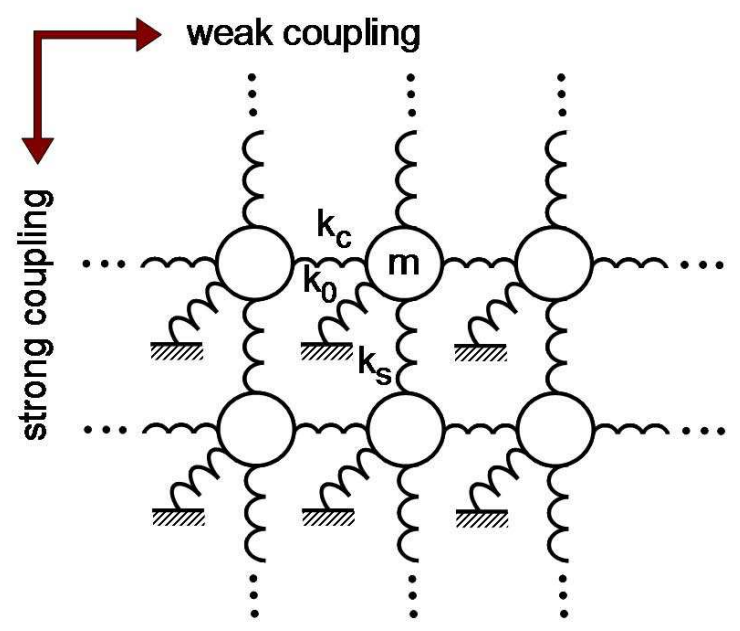

Figure 2.25: 2D mechanical coupling configuration under investigation to reduce effective resonator variations. Here, the coupling stiffness $k_{C} \ll k_{S}$.

$$
\sigma_{e f f}=\frac{\sigma}{\sqrt{\frac{N_{S}+1}{2}}}, N_{S} \text { odd }
$$

The enhanced number of acoustic energy paths in the filter provided by the strong coupling reduces the effect of resonator variations, averaging out stiffness mismatch between resonators, consequently improving passband distortion.

\subsubsection{Experimental Setup}

\section{Filter Design}

In this study, four resonator coupling configurations are investigated to determine the effectiveness of the 2D strongly coupled array filter. We construct a 1D 4-pole filter (Figure 2.26a) as a basis of comparison for all 2D filters in the 
study. The performance of this 1D filter is compared with a set of four 1D 4pole filters, electrically summed in parallel (Figure 2.26b), a 2D 4x4 array of resonators, weakly coupled in both directions (Figure 2.26c), and a 2D 4x4 array of resonators, coupled weakly in one direction and strongly in the other (Figure $2.26 \mathrm{~d})$.

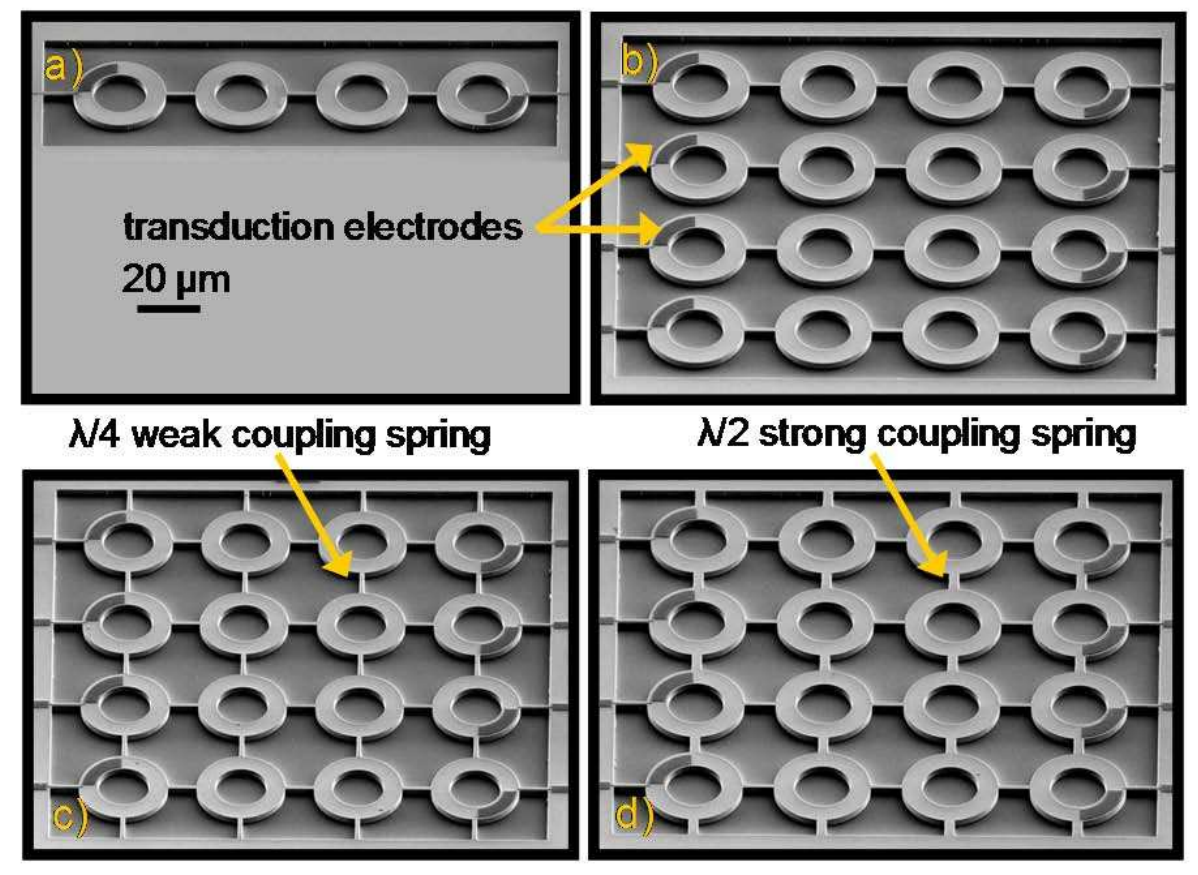

Figure 2.26: Scanning electron micrographs of (a) a 1D 4-pole filter, (b) 4 electrically summed 1D 4-pole filters, (c) a $4 \times 4$ array of resonators, coupled weakly in both directions, and (d) a $4 \times 4$ array of resonators, coupled weakly in one direction and strongly in the other.

The filters are composed of extensional wine glass ring resonators [21] with a fundamental resonance designed for $500 \mathrm{MHz}$. The resonators are driven and sensed with lateral dielectric transduction. Soft coupling springs between the resonators, used to define the bandwidth of the filter, are achieved with $2 \mu \mathrm{m}$ wide beams of quarter wave length matched at $500 \mathrm{MHz}$. Strong coupling 
springs used in the proposed strongly coupled array are designed with $4 \mu \mathrm{m}$ wide beams of half wave length. The resonators are coupled at the quasi-nodes of the extensional wine glass ring mode.

The choice of a resonant mode with $90^{\circ}$ rotational symmetry (albeit out-ofphase) enables an ideal 2D coupling for this study. The effective stiffness of the resonator at the coupling point is identical for coupling in both directions, such that the coupling is defined nominally by the dimensions of the coupling beam. This design is chosen for ease of comparison across several filters. However, the restriction on resonator type is not a requirement, and any resonator can be implemented with proper modelling.

\section{Fabrication}

The filters are fabricated in a simple SOI process for lateral dielectric transduction, shown in Figure 3. First, $100 \mathrm{~nm}$ of LPCVD stoichiometric silicon nitride are deposited on a $3 \mu m$ thick $\mathrm{n}+$ silicon device layer. This dielectric forms the transduction film for the resonators. The nitride is then patterned to provide electrical contact to the device layer for biasing the resonators. $100 \mathrm{~nm}$ of LPCVD $\mathrm{n}+$ polysilicon are then deposited and patterned to form the probe pads, routing, and transduction electrodes. The resonator bodies are then defined in a deep reactive ion etch (DRIE) of the silicon device layer. Finally, the resonators are released in HF and critical-point dried (CPD) to prevent stiction. 


\subsubsection{Experimental Results}

\section{Measurement And Calibration}

The filters were characterized in a vacuum RF probe station in a 2-port configuration using GSG probes. Parasitics up to the probe tips were first cancelled with short-open-load-through (SOLT) measurements on a standard calibration substrate. De-embedding was then performed with Cascade WinCal software, using short, open, and through structures fabricated on-chip, but separate from the filters. This de-embedding allows for the cancellation of the large pad capacitance without cancelling out any parasitics inherent to the filters themselves, including suspension beam routing and transduction electrodes on the resonators.

\section{Wine Glass Ring Resonator}

Figure 2.27 shows the $50 \Omega$ terminated $S_{21}$ response of a single wine glass ring resonator, applying a bias voltage of $11 \mathrm{~V}$. Ten independent resonators were characterized in vacuum, varying in frequency from 511-514 MHz and in electromechanical quality factor $\left(Q_{e m}\right)$ from 5000 to 8000 .

\section{Filter Performance}

Applying $10 \mathrm{~V}$ across the $100 \mathrm{~nm}$ nitride transducer, we obtain the frequency response of the 1D 4-pole filter, shown in Figure 2.28a. The filter has a center frequency of $511 \mathrm{MHz}$ with a $3 \mathrm{~dB}$ bandwidth of $1.49 \mathrm{MHz}$. The $50 \Omega$ terminated response clearly shows the four poles defining the filter. Due to fabrication variations, there is a significant distortion to the passband, with a ripple (defined as 


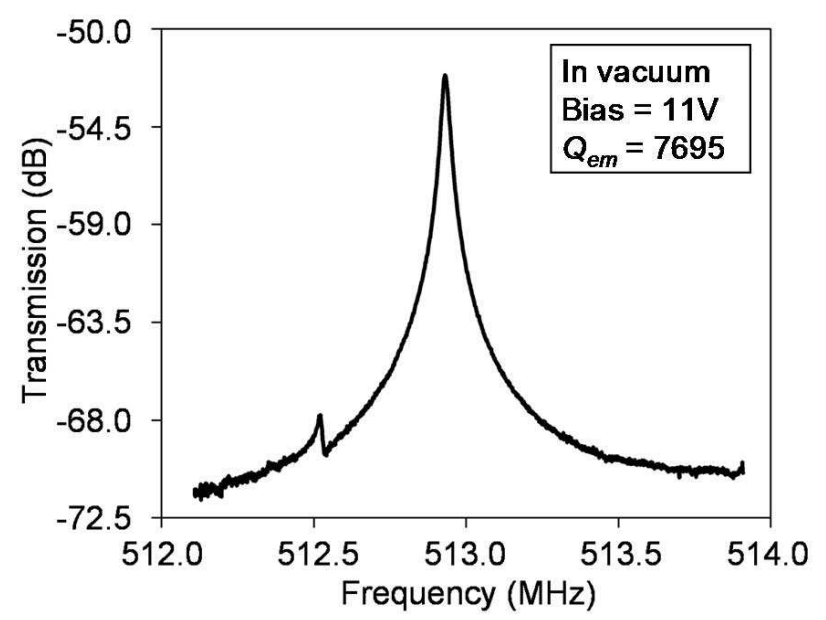

Figure 2.27: $50 \Omega$ terminated $S_{21}$ transmission response of a single wine glass ring resonator.

the maximum peak to minimum trough) of $5.4 \mathrm{~dB}$. The $1 \mathrm{D}$ filter frequency response provides a basis of comparison for the rest of the filters in this study.

Figure $2.28 \mathrm{~b}$ presents the $50 \Omega$ terminated S21 transmission of the 4 electrically summed 1D 4-pole filters (Figure 2.26b). The increased transduction area of the electrically summed 1D filters improved the insertion loss (IL, defined here at the maximum peak) by $7 \mathrm{~dB}$ relative to the single $1 \mathrm{D}$ chain. Additionally, the passband flattened to only $1.1 \mathrm{~dB}$ ripple, due to the summation of four 4-pole filters, offset in frequency from one another due to fabrication variations. However, these improvements are at the expense of filter shape factor and stopband rejection. The electrically summed array filter has a $3 \mathrm{~dB} 8 \mathrm{~dB}$ shape factor of 2.19 a 30\% degradation from the single 1D chain shape factor of 1.68. Furthermore, the stop-band rejection of the electrically summed filter reduces to $11.1 \mathrm{~dB}$ from $16.7 \mathrm{~dB}$ in the case of the single chain.

We next inspect the case of the $4 \times 4$ array of resonators, weakly coupled in both directions, shown in Figure 2.28c. This 2D array has too many resonant modes to be considered a filter. However, the behavior of this filter demon- 
strates that the strong coupling in the next filter is indeed strong enough relative to the weakly coupled direction.

Finally, we observe the effects of coupling a $4 \times 4$ 2D array of resonators weakly in one direction (defining the resonant modes which contribute to the passband) and strongly in the other direction (averaging out fabrication variations). The $50 \Omega$ terminated frequency response of this filter is presented in Figure 2.28d. As in the case of the electrically summed filters, the insertion loss improves due to increased transduction area. It should be noted that the expected improvement in IL for both the electrically summed filter and the strongly coupled 2D filter is $13.9 \mathrm{~dB}$ due to the $4 \mathrm{x}$ increase in electrode area. However, the filters show an IL increase of only 7-8 dB. This discrepancy is attributed to the process variations, including non-uniformity of the nitride thickness and polysilicon resistivity.

It should be noted that the bandwidth of the strongly coupled filter (1.1 $\mathrm{MHz})$ is narrower than that of its $1 \mathrm{D}$ counterpart $(1.49 \mathrm{MHz})$. The bandwidth narrowing corresponds to an effective stiffening of the resonator due to the nonideal strong coupling beams. It can be compensated using different strong coupling designs (dependent on the geometry of the resonators comprising the filter) or simply by changing the effective stiffness of the soft coupling beams in the array.

Additionally, there is a frequency shift in the passband of about $1 \mathrm{MHz}$ relative to the $1 \mathrm{D}$ filter. This frequency shift can be observed in the frequency response of all the 2D arrays, and is attributed variations in DRIE device layer etch rates for small and large open areas on a single mask. In the case of the $2 \mathrm{D}$ strongly coupled array, the center frequency shift is due to both etch rate effects and due to resonator mass loading from the $4 \mu m$ wide strong coupling beams. 

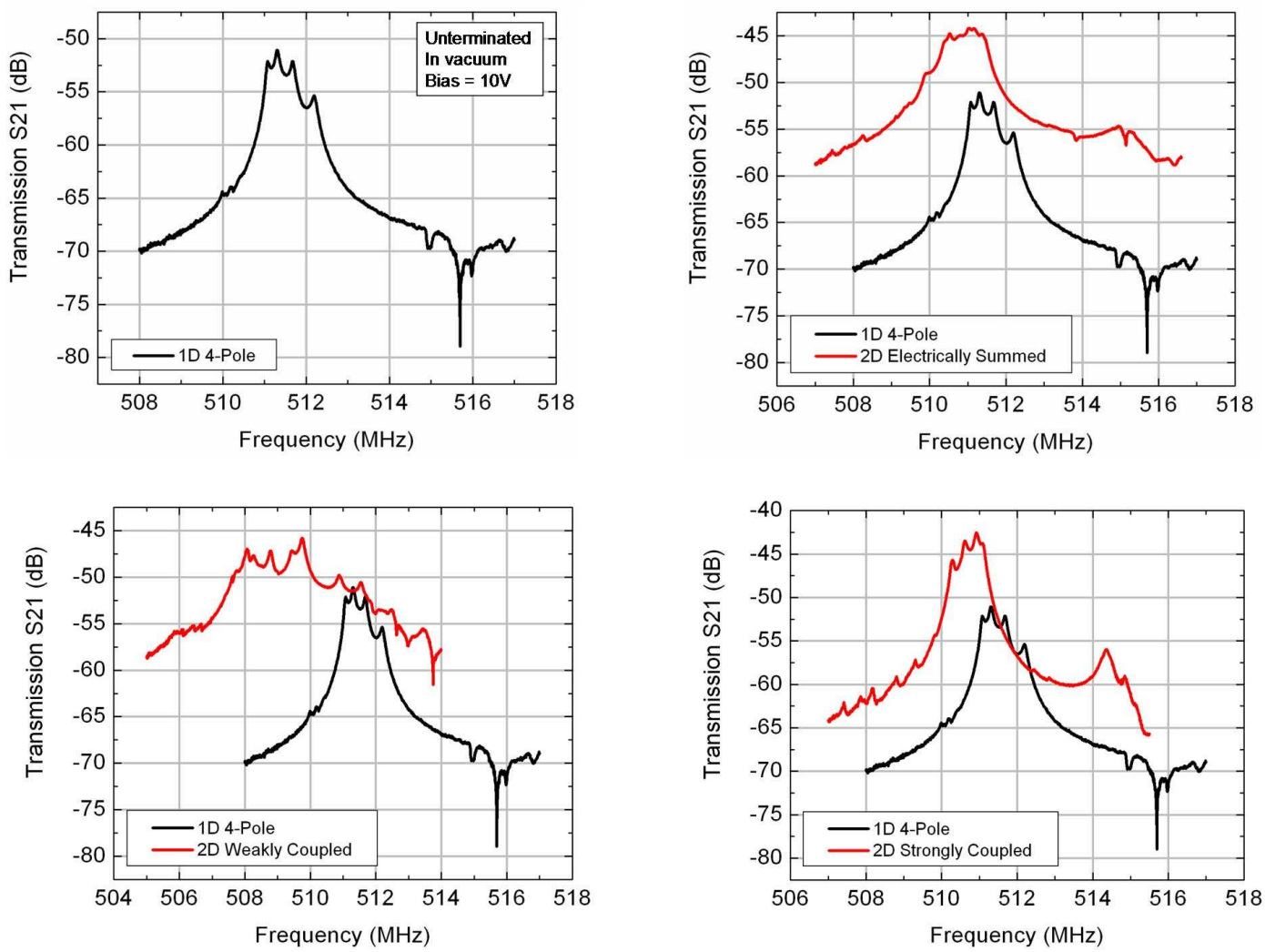

Figure 2.28: $50 \Omega$ terminated $S_{21}$ transmission plot of (a)1D 4-pole filter, (b) comparison of 1D filter with 4 electrically summed 1D filters, (c) comparison of 1D filter with $4 \times 42 \mathrm{D}$ weakly coupled array, and (d) comparison of 1D filter with 4x4 2D strongly coupled array.

The ripple of the $2 \mathrm{D}$ strongly coupled filter improves from $5.4 \mathrm{~dB}$ to $4.2 \mathrm{~dB}$ relative to the 1D 4-pole filter. This corresponds to a $22 \%$ improvement in the passband ripple.

The improvement in passband distortion does not sacrifice filter stop-band rejection and shape factor. The stop-band rejection increases from $16.7 \mathrm{~dB}$ to 17.6 $\mathrm{dB}$ while the filter shape factor $(3 \mathrm{~dB} 8 \mathrm{~dB})$ decreases from 1.68 to 1.55 for the $2 \mathrm{D}$ strongly coupled filter relative to the 1D filter. These 5-8\% improvements do not make the observed trend definitive. A larger number of 2D strongly cou- 
pled filters must be tested in order to determine if the strong coupling configuration actually improves these filter characteristics. It is evident, however, that the $2 \mathrm{D}$ coupling improves distortion in the filter passband without degrading filter performance.

\subsubsection{Conclusion}

This study of 2D resonator arrays demonstrated the effectiveness of a 2D mechanical coupling configuration for filters in reducing passband distortion due to micro-fabrication variations. A 2D filter comprised of a $4 \times 4$ array of bulkmode wine glass ring resonators was demonstrated at $511 \mathrm{MHz}$. The 2D coupling provided a $22 \%$ improvement in $50 \Omega$ terminated passband ripple relative to its $1 \mathrm{D}$ counterpart, without degradation in stop-band rejection or shape factor. This strong mechanical coupling provides a more robust solution to fabri-

Table 2.1: Summary Of $50 \Omega$ Terminated Filter Performance

\begin{tabular}{|l|l|l|l|}
\hline \hline Parameter & $\begin{array}{l}\text { 1D 4-Pole } \\
\text { Filter }\end{array}$ & $\begin{array}{l}\text { 2D Electronically } \\
\text { Summed Filter }\end{array}$ & $\begin{array}{l}\text { 2D Strongly } \\
\text { Coupled Filter }\end{array}$ \\
\hline Insertion Loss & $-51.1 \mathrm{~dB}$ & $-44.2 \mathrm{~dB}$ & $-42.5 \mathrm{~dB}$ \\
3dB Bandwidth & $1.49 \mathrm{MHz}$ & $1.56 \mathrm{MHz}$ & $1.1 \mathrm{MHz}$ \\
Stop-Band Rejection & $16.7 \mathrm{~dB}$ & $11.1 \mathrm{~dB}$ & $17.6 \mathrm{~dB}$ \\
Shape Factor (3dB-8dB) & 1.68 & 2.19 & 1.55 \\
Ripple & $5.38 \mathrm{~dB}$ & $1.18 \mathrm{~dB}$ & $4.22 \mathrm{~dB}$ \\
\hline
\end{tabular}

cation variations than the electrically summed filter, which suffered a 33\% decrease in stop-band rejection and a $30 \%$ increase in shape factor relative to the 
1D filter. For reference, Table 2.1 quantifies the transmission response of the three filters under examination.

In addition, the electrically summed filter demonstrates unpredictable bandwidth from filter to filter, due to its sensitive dependence on fabrication variations. Though the electrically summed filter produced a flatter passband than the strongly coupled filter in the case of the $4 \times 4$ array, we can simply couple more resonators in the stiff direction to improve passband distortion and insertion loss, without degradation of filter performance. The 2D strong mechanical coupling configuration examined in this work can be implemented with any resonators in any fabrication process, providing more reliable and repeatable high-performance MEMS filters. 


\section{CHAPTER 3}

\section{INTERNAL DIELECTRIC TRANSDUCTION: THEORY}

\subsection{Motivation}

Extending the frequency of MEMS resonators generally entails scaling of resonator dimensions leading to increased motional impedance. Most electrostatic MEMS resonators to date employ air-gap capacitive transduction to drive and sense resonant motion. Dielectric electrostatic transduction has several benefits over common air-gap transduction; it is desirable in order to achieve smaller capacitive gaps, to prevent pull-in and stiction symptomatic of air-gap transducers, and to enhance driving force and capacitive sensing due to high dielectric permittivity. Dielectrics can therefore extend resonant frequencies to the $>5$ $\mathrm{GHz}$ range, where these issues are most prominent. However, most devices demonstrated to date are geometrically identical to their air-gap counterparts, with a dielectric film in place of the air-gap transducer. As resonators scale to higher frequencies and smaller dimensions, this transduction configuration may not be most suitable. This work focuses on scaling electrostatic acoustic resonators to the SHF and EHF bands of the radio spectrum. Resonator applications in this frequency range include microwave oscillators, with particular emphasis on low-power clocking in microprocessors.

This chapter presents the theory of internal dielectric transduction (IDT) of longitudinal bulk mode MEMS resonators. This transduction mechanism increases in efficiency as the dielectric thickness approaches the acoustic halfwave length in silicon, enabling the realization of MEMS resonators at multiGHz frequencies. The underlying difference between internal and external di- 
electric transduction determines their capabilities at higher frequencies. Both mechanisms employ dielectric drive and sense transducers. External transduction assumes free boundary conditions (zero stress) at the dielectric interface, driving at a frequency corresponding to a resonant mode with maximum displacement at the dielectric, and necessitating maximum acoustic mismatch between the dielectric and resonator bulk. This condition is an extension of the case of air-gap transducers, in which the acoustic mismatch approaches infinity in vacuum. The boundary condition at the dielectric interface results in an observed transduction loss, particularly at higher frequencies.

On the other hand, internal transduction incorporates the dielectric film into the resonant mode shape. This generally involves the assumption of a close acoustic match between the bulk resonator and dielectric film. In practice, a mismatch in acoustic impedance between the dielectric and resonator material results in a shift of the resonant frequency, and can easily be compensated by altering the dimensions of the resonator.

\subsection{Analysis: Capacitive Drive And Sense}

A longitudinal-mode bar resonator is driven and sensed electrostatically with thin vertical dielectric layers, as shown in Figure 3.1a. The resonator body is biased to $V_{D C}$, and a harmonic excitation of amplitude $v_{i n}$ is applied to the drive electrode at resonant frequency. Internal transduction requires that the dielectric films be acoustically matched to the bulk resonator material, thereby maintaining the mode shape and frequency of the resonator without degrading the

quality factor. With this assumption, the nth harmonic of the free-free longitu- 


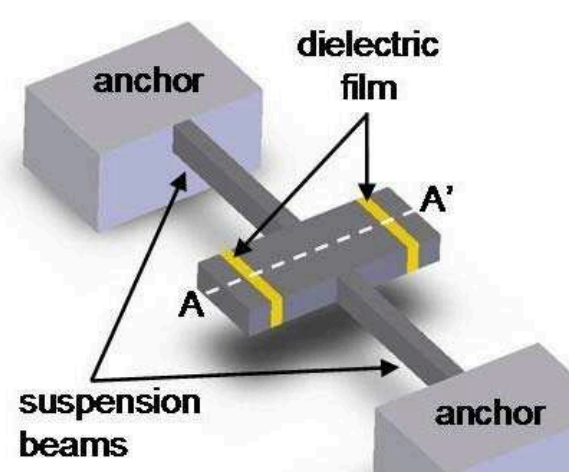

(a)

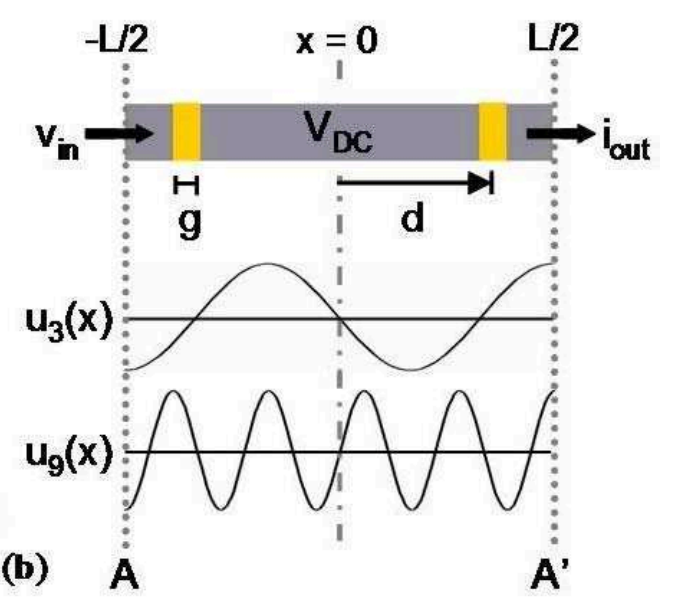

Figure 3.1: (a) Schematic of dielectrically transduced free-free longitudinal bulk mode resonator. The dielectric films are incorporated into the resonator, driving and sensing electrostatically. (b) Cross section of bar resonator. A bias voltage $V_{D C}$ is applied to the resonator. An AC voltage $v_{\text {in }}$ on one end drives resonance, while an output current $i_{\text {out }}$ is measured at the other. The normalized amplitudes of the 3rd and 9th longitudinal mode harmonics are displayed.

dinal mode bar spanning $\frac{-L}{2} \leq x \leq \frac{L}{2}$ has displacement following

$$
u(x, t)=U_{0} e^{i 2 \pi f_{n} t} \sin \left(k_{n} x\right), \quad \mathrm{n} \text { odd },
$$

where $k_{n}=n \pi / L$ and $U_{0}$ is the maximum amplitude of vibrations of the bar. Figure $3.1 \mathrm{~b}$ illustrates the $3 \mathrm{rd}$ and 9 th harmonics of this longitudinal mode. The resonant frequency of the nth harmonic is $f_{n}=(n / 2 L) \sqrt{Y / \rho}$ for $Y$ and $\rho$ the Youngs 
modulus and mass density of the bar, respectively. The driving dielectric film of thickness $g$ is placed at in the resonator. The AC component of the capacitive force across the dielectric of permittivity $\varepsilon_{f}$ is

$$
f(x, t)=\frac{\varepsilon_{f} A}{g^{2}} V_{D C} v_{i n} e^{i 2 \pi f_{n} t} \quad \forall x \in\left[d-\frac{g}{2}, d+\frac{g}{2}\right]
$$

Given the equation of motion for damped vibrations in a bar [27],

$$
\rho A \frac{\partial^{2} u(x, t)}{\partial t^{2}}-b A \frac{\partial^{3} u(x, t)}{\partial t \partial x^{2}}-Y A \frac{\partial^{2} u(x, t)}{\partial x^{2}}=\frac{\partial f(x, t)}{\partial x}
$$

and substituting equation 1 into equation 3 , the amplitude of vibrations at resonant frequency is given by

$$
U_{0}=\frac{2 Q \varepsilon_{f} V_{D C} v_{\text {in }}}{n^{2} \pi^{2} Y} \frac{L}{g^{2}}\left[\sin \left(k_{n} d-\frac{k_{n} g}{2}\right)-\sin \left(k_{n} d+\frac{k_{n} g}{2}\right)\right]
$$

for $Q$ the quality factor of the resonator. This resonance is detected by the changing capacitance due to vibrations at the sensing dielectric film,

$$
\begin{aligned}
i_{\text {out }} & =V_{D C} \frac{d C}{d t} \\
& =V_{D C} \frac{d C}{d u} \frac{d u}{d t} \\
& =\frac{\varepsilon_{f} V_{D C} A}{g^{2}}\left[\sin \left(k_{n} d-\frac{k_{n} g}{2}\right)-\sin \left(k_{n} d+\frac{k_{n} g}{2}\right)\right] 2 \pi f_{n} U_{0} \\
& =\frac{2 Q \varepsilon_{f}^{2} V_{D C}^{2} A}{n \pi \sqrt{Y \rho} g^{4}}\left[\sin \left(k_{n} d-\frac{k_{n} g}{2}\right)-\sin \left(k_{n} d+\frac{k_{n} g}{2}\right)\right]^{2} v_{\text {in }}
\end{aligned}
$$


resulting in a motional impedance

$$
\begin{aligned}
R_{X} & \equiv \frac{v_{\text {in }}}{i_{\text {out }}} \\
& =\frac{n \pi \sqrt{Y \rho}}{2 Q A \varepsilon_{f}^{2} V_{D C}^{2}} \frac{g^{4}}{\left[\sin \left(k_{n} d-\frac{k_{n} g}{2}\right)-\sin \left(k_{n} d+\frac{k_{n} g}{2}\right)\right]^{2}}
\end{aligned}
$$

simplifying to

$$
R_{X}=\frac{n \pi \sqrt{Y \rho}}{8 Q A \varepsilon_{f}^{2} V_{D C}^{2}} \frac{g^{4}}{\cos ^{2}\left(k_{n} d\right) \sin ^{2}\left(k_{n} g / 2\right)}
$$

Equation 3.7 provides important guidelines for optimizing the performance of bulk-mode resonators using internal dielectric transduction. As expected, the quartic dependence of the motional impedance on dielectric thickness necessitates the thinnest dielectric possible. This is generally limited by fabrication and material properties. Furthermore, this form for the motional impedance, differing from air-gap transduction primarily by the trigonometric terms in the denominator, indicates that the position of both drive and sense dielectric films should be centered at a displacement minimum, or strain maximum. This choice for the position of the dielectric films sets $\cos ^{2}\left(k_{n} d\right)=1$, minimizing $R_{X}$ with respect to $d$.

The $\sin ^{2}$ term in the denominator of Equation 3.7 results from the modal displacement at the dielectric-bulk resonator interface. As noted in [28], this factor degrades the performance of the resonator considerably at low frequencies, where the acoustic wavelength $\lambda \gg g$. However, as the resonator scales to higher frequencies, and $\lambda / 2 \rightarrow g$, the $\sin ^{2}$ term in the denominator approaches unity, reducing motional impedance. Consequently, for a fixed dielectric thickness determined by fabrication limitations, there is an optimal frequency of operation with acoustic wavelength $\lambda=2 g$. 
Figure 3.2 presents the $R_{X}$ of the 3rd and 9th harmonics of an internally transduced longitudinal bar, varying the dielectric position along the length of the resonator. A constant $f . Q$ product of $5 \times 10^{13}$ is assumed for both harmonics, limited by thermoelastic dissipation and Akhieser effect in longitudinal bulkmode resonators. As shown in the plot, $R_{X}$ minima in the motional impedance occur for points of maximum strain (minimum displacement). The large spatial range near the displacement nodes over which $R_{X}$ is low allows for fabrication of reliable devices despite misalignment tolerances. The coincidence of displacement nodes of the 3rd and 9th harmonics at the fractional dielectric position of $2 / 3$ allows for the optimal excitation of both modes in the same device. This may be useful for multi-frequency applications. However, if multiple modes are undesired, the 3rd harmonic can be suppressed by placing the dielectric at a fractional dielectric position of 2/9 or 4/9, near a displacement maximum of the 3rd harmonic, while still driving the 9th harmonic at maximum strain.

Frequency scaling of the bulk mode longitudinal resonators using internal dielectric transduction is shown in Figure 3.3. The motional impedance, normalized to the cross-sectional area of the resonator, decreases drastically with increasing frequency, achieving $10 \mathrm{k} \Omega . \mu \mathrm{m}^{2}$ impedances at $60 \mathrm{GHz}$. Again, a constant $f . Q$ product of $5 \times 10^{13}$ is assumed. The frequency scaling result of Fig. 3 converges to an FBAR-like resonator or the Bragg reflector for a solid-mounted BAW resonator, stacking multiple dielectrics of thickness $\lambda / 2$ between conductive layers of the same thickness. Recently, such devices have been demonstrated successfully in the $10 \mathrm{GHz}$ range [29].

Common dielectrics such as silicon dioxide $(\kappa=3.9)$ and silicon nitride $(\kappa=7)$ perform reliably in films as thin as a few nanometers. For such transduction film thickness, the motional impedance is minimized at $>50 \mathrm{GHz}$, but 


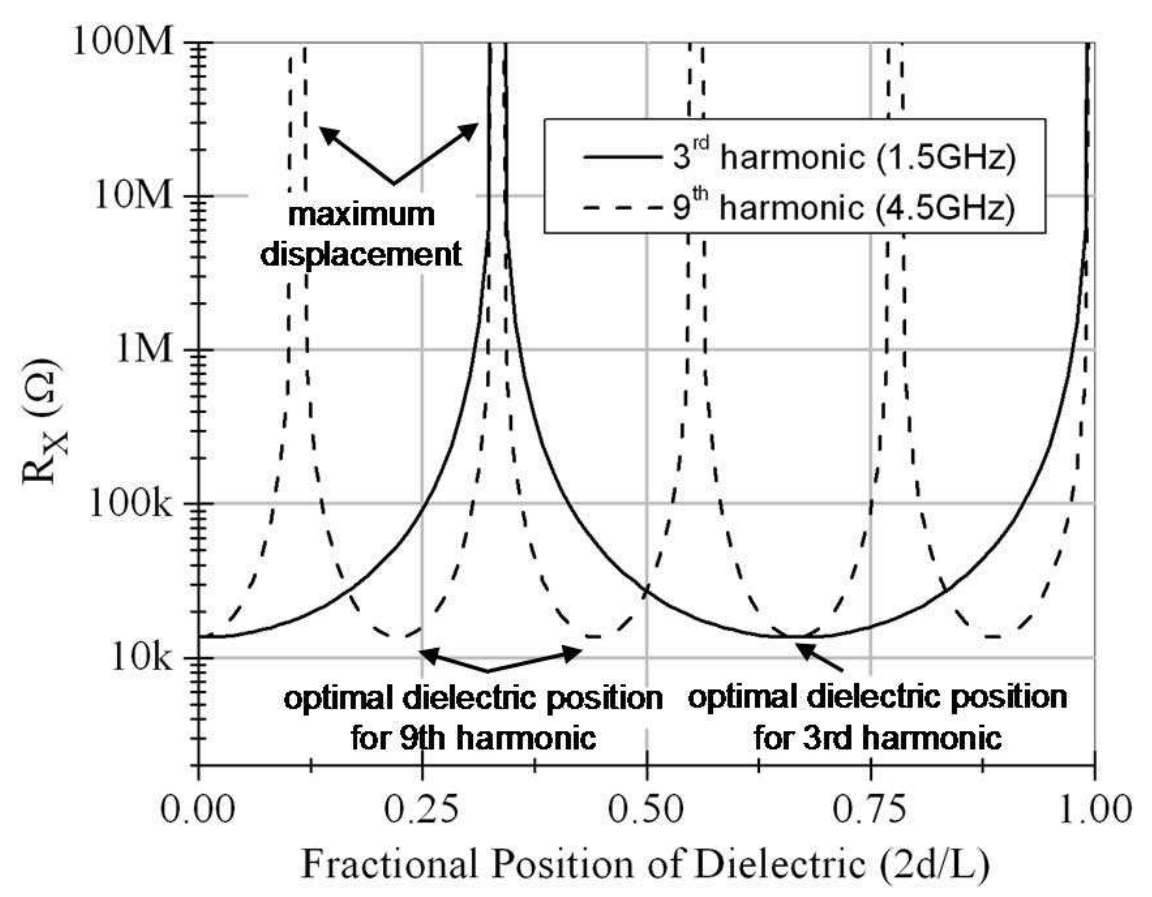

Figure 3.2: $R_{X}$ as a function of fractional dielectric position $\left|\frac{2 d}{L}\right|$ for the 3rd and 9th harmonic longitudinal (width-extensional) modes of a $8.5 \mu \mathrm{m}$ long bar. $\mathrm{g}=15 \mathrm{~nm}$, resonator thickness is $2.5 \mu \mathrm{m}$, width is $40 \mu \mathrm{m}, V_{D C}=10 \mathrm{~V}, \varepsilon_{f}=7 \varepsilon_{0}$, and an $f Q$ product of $5 \times 10^{13}$ is assumed.

may be too high for 1-10 GHz operation. Low impedance resonators in the radio and microwave frequency range can be achieved by using high- $\kappa$ dielectric materials, such as Barium Strontium Titanate (BST). While BST films are not electrically reliable below $\sim 200 \mathrm{~nm}$, they exhibit a high permittivity often exceeding 300. This will prove to be a great advantage in obtaining low-impedance internally transduced resonators at low-GHz frequencies. Minimizing Equation 3.7 with respect to resonant frequency for a $200 \mathrm{~nm}$ dielectric film, one obtains an optimal frequency of operation at $15.8 \mathrm{GHz}$. Assuming an $f . Q$ product is $5 \times 10^{13}$ and a bias voltage of $20 \mathrm{~V}$, this structure has $5 \mathrm{k} \Omega . \mu \mathrm{m}^{2}$ impedance at 3rd harmonic resonance. For instance, a $50 \Omega$ BST resonator at $15 \mathrm{GHz}$ can be obtained by stacking the bulk/dielectric layers vertically (thickness extensional mode) 


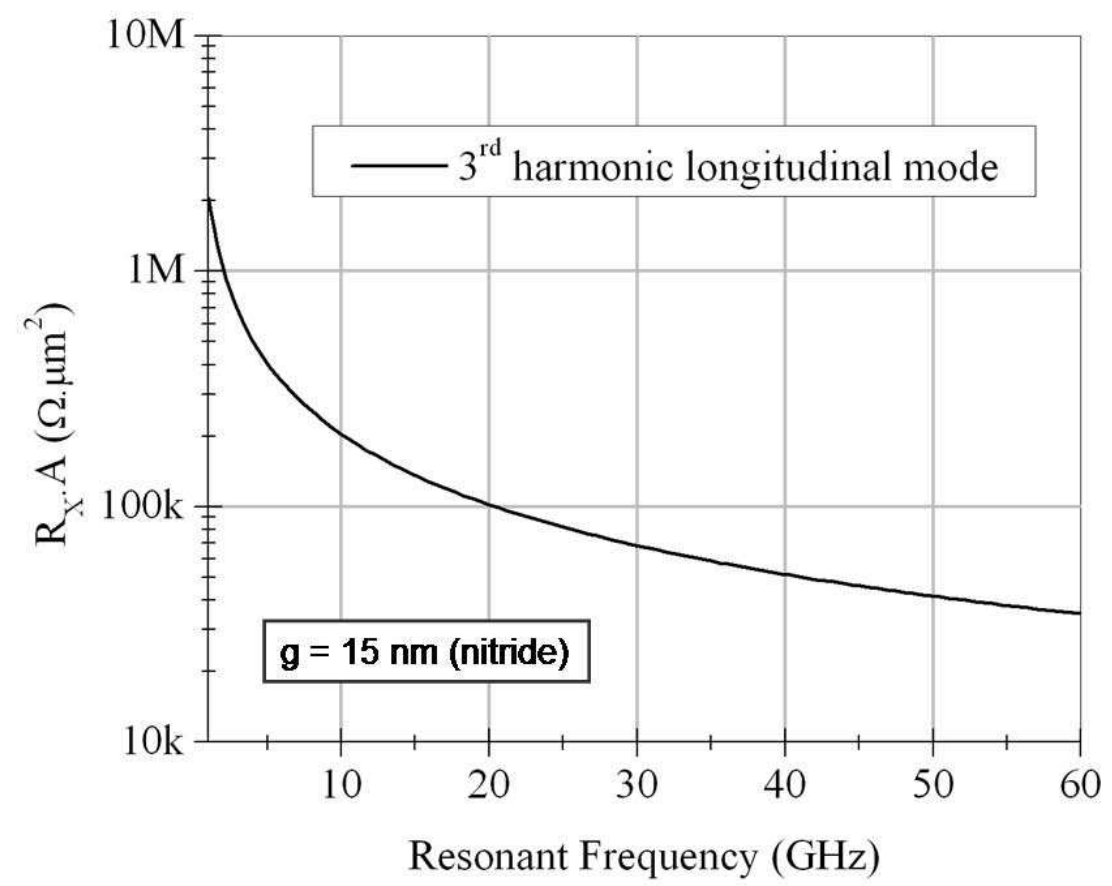

Figure 3.3: $R_{X}$ scaling with frequency, normalized to the cross-sectional area of the resonator. $\mathrm{g}=15 \mathrm{~nm}, V_{D C}=10 \mathrm{~V}$, and $\varepsilon_{f}=7 \varepsilon_{0}$. The dielectric films are placed at maximum strain. An $f . Q$ product of $5 \times 10^{13}$ is assumed.

with a $10 \mu m \times 10 \mu m$ footprint, or by forming a $1 \mu m$ thick extensional ring [30] with an approximate radius of $16 \mu \mathrm{m}$.

\subsection{Analysis: Electrostriction}

In addition to the parallel-plate capacitive force driving resonance as described in $\S 3.2$, there exists a time-varying internal strain in the dielectric drive transducer due to electrostriction. Electrostriction is a property of dielectrics associated with randomly oriented domains within the material. In the presence of an electric field, these domains form localized polarizations which can attract each 
other, causing a strain to form in the dielectric material.

As an example of the effects of electrostriction, let us take the case of a silicon nitride dielectric transducer. The electrostrictive constant in nitride is $Q=1.06 \mathrm{~m}^{4} / \mathrm{C}^{2}$. This results in a longitudinal strain inside the dielectric

$$
\begin{aligned}
\varepsilon_{e s} & =Q P^{2} \\
& =Q(1-\kappa)^{2} \varepsilon_{0}^{2} E^{2} \\
& =3.0 \times 10^{-21}\left(\frac{V}{g}\right)^{2} .
\end{aligned}
$$

$P$ is the polarization, $k$ is the relative permittivity of the dielectric, $E$ is the applied electric field, $V$ is the applied voltage, and $g$ is the dielectric thickness [31]. For $V=V_{D C}+v_{i n} e^{i \omega t}$, the component of the strain oscillating at $\omega$ is

$$
\left.\varepsilon_{e s}\right|_{\omega}=6.0 \times 10^{-21}\left(\frac{V_{D C} v_{i n}}{g^{2}}\right) .
$$

The strain induced by electrostatic force in IDT devices is

$$
\begin{aligned}
\varepsilon_{\text {cap }} & =U_{0} k_{n} \\
& =1.7 \times 10^{-18}\left(\frac{V_{D C} v_{\text {in }}}{g^{2}}\right) \sin \left(1.7 \times 10^{6} \mathrm{~g}\right) \\
& =2.8 \times 10^{-12}\left(\frac{V_{D C} v_{\text {in }}}{g}\right) .
\end{aligned}
$$

For a $15 \mathrm{~nm}$ thick film,

$$
\frac{\varepsilon_{c a p}}{\varepsilon_{e s}} \approx 7.1
$$

The electrostriction of the nitride enhances transduction efficiency by increasing the effective force driving resonance at frequency $\omega$. Though silicon nitride and 
other CMOS-compatible materials have low electrostrictive constants, special dielectrics can be implemented where possible to take advantage of this effect. 
CHAPTER 4

INTERNAL DIELECTRIC TRANSDUCTION: FABRICATION

\subsection{IDT Devices At The Cornell Nanoscale Facility}

The resonators were fabricated in a combined SOI-polysilicon process using a $15 \mathrm{~nm}$ silicon nitride film for transduction, as detailed in Figure 4.1. (1) The device layer of an SOI wafer is first patterned in DRIE with a hard oxide mask. The device layer is $2.5 \mu \mathrm{m}$ thick. (2) A $15 \mathrm{~nm}$ conformal LPCVD silicon nitride film is then deposited to form the transduction dielectric. (3) A layer of $n+$ polysilicon $>3 \mu \mathrm{m}$ thick is deposited, annealed, and (4) smoothed with CMP. (5) A second hard oxide mask is then deposited and patterned to (6) etch the polysilicon and define the final resonator shape. (7) The resonators are released in an HF timed etch followed by a critical-point dry (CPD) step to prevent stiction. Though the outer rim of silicon nitride is removed in the HF release step (Figure 4.2 inset), the nitride remains in the majority of the transduction area as evidenced by capacitive measurements.

Suspension beams for the resonators are designed at quarter-wave length to minimize anchor losses for both 3rd and 9th harmonics and dampen spurious modes. The mode shape and SEM of the resonator are presented in Figure 4.2. As seen in the figure, the non-ideal routing beams of the input and output electrodes of the resonator distort the one-dimensional longitudinal mode shape assumed for transduction calculations and frequency scaling. The distorted mode, which couples an 8th harmonic surface mode to the original longitudinal resonance, slightly degrades the transduction efficiency due to a small cancellation in signal from the summed contribution of both tensile and compressive strain 
in the dielectric film. However, the primary contribution to the capacitive sensing results from the longitudinal component of the dielectric strain, yielding transduction close to that described by the theory in the previous chapter.

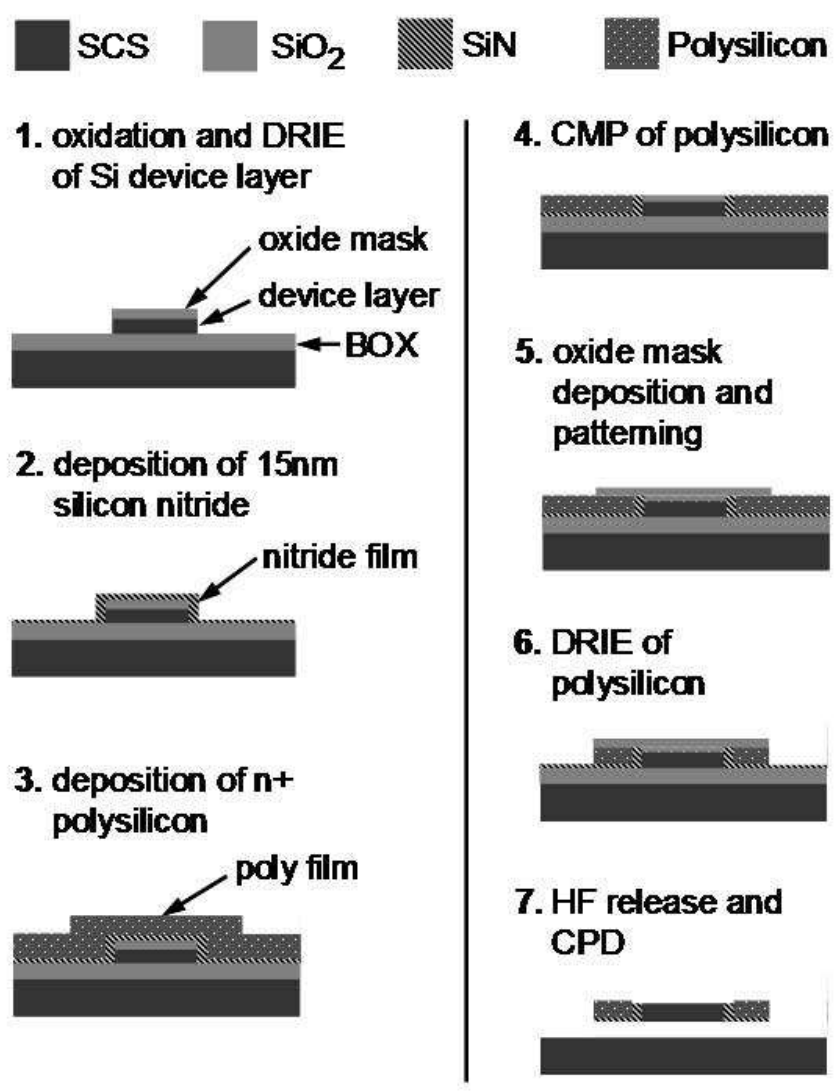

Figure 4.1: Micro-fabrication process for internal dielectric transduced resonators. 


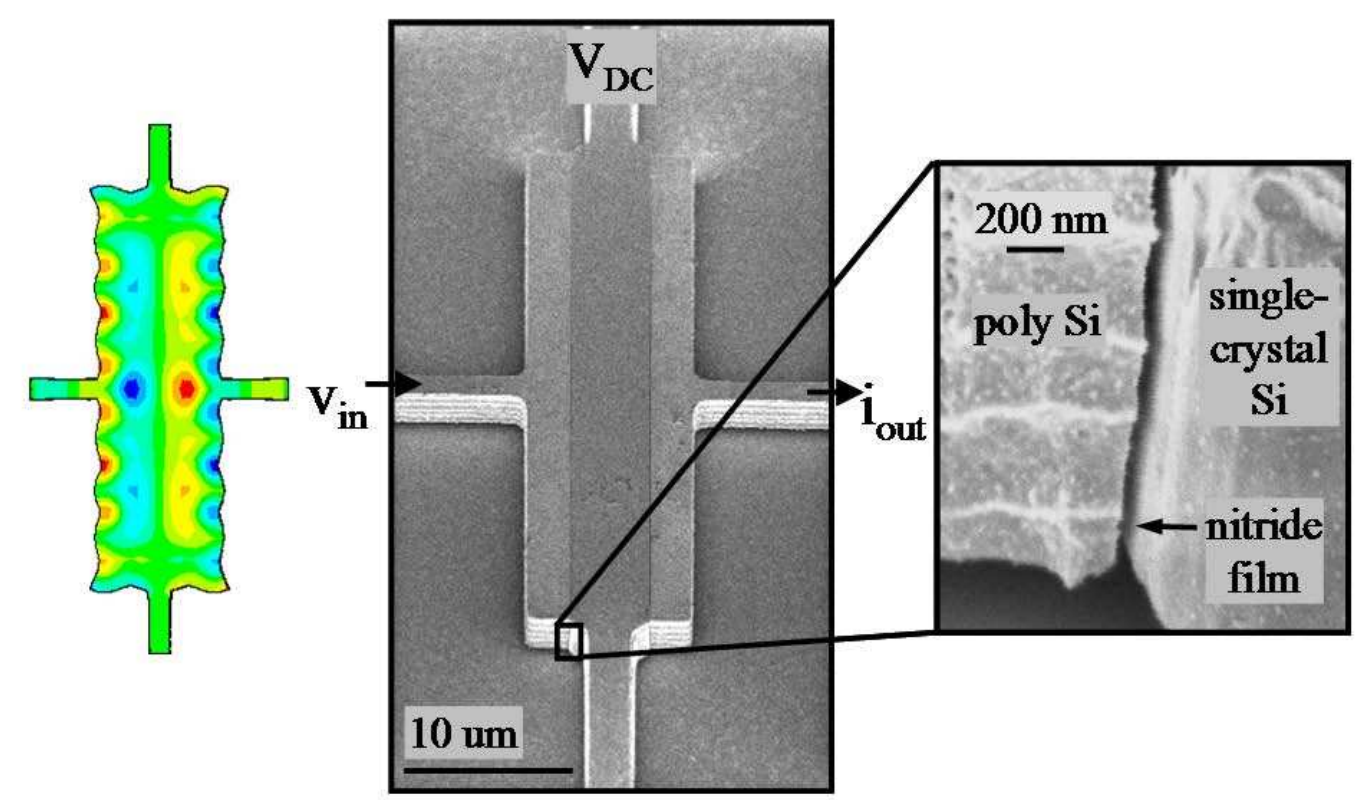

Figure 4.2: Left: Modal analysis of 3rd harmonic resonant mode shape of the bar resonator, simulated in Ansys. Right: Scanning Electron Micrograph of a dielectrically transduced silicon bar resonator $(8.5 \mu \mathrm{m}$ long $\times 40 \mu \mathrm{m}$ wide $\times 2.5 \mu \mathrm{m}$ tall). The inset image shows the thin gap between the polysilicon and single crystal regions of the resonator.

\subsection{IDT Devices At The Sony Nanotechnology Development}

\section{Department}

To obtain high-yield fabrication of IDT bar resonators, we collaborated with Sony Nanotechnology Development Department in Japan to develop a surface micromachining process. Working with Dr. Koichi Ikeda's group at Sony, we developed a IDT fabrication process outlined in Figure 4.3. This process is similar to the one executed at Cornell, but eliminates the need for SOI wafers. 
A blank Silicon wafer is coated with a $200 \mathrm{~nm}$ film of doped Polysilicon (Poly-Gnd) to ensure a conductive substrate for the devices. A $1 \mu \mathrm{m}$ thick lowpressure TEOS oxide (Ox-Sub) is then deposited for electrical isolation of the device from the substrate. This is followed by a $200 \mathrm{~nm}$ film of Silicon Nitride (SiN1), which serves as an etch stop during HF release of the devices. Contact holes are then etched through SiN1 and Ox-Sub to allow for electrical connectivity to the substrate Poly-Gnd. A $400 \mathrm{~nm}$ layer of CVD Polysilicon (Poly-0) is then deposited and patterned to form ground planes and routing to the device (Figure 4.3a). Following definition of the Poly-0 layer, a $500 \mathrm{~nm}$ TEOS oxide layer (OX-1) is deposited as a sacrificial oxide layer and patterned to define anchors and routing to Poly- 0 .

A $2 \mu \mathrm{m}$ thick layer of Polysilicon (Poly-1) is then deposited and patterned using a hard oxide mask (OX-2) to define the position of the dielectric transducer within each resonator (Figure 4.3b). A 15-20 nm conformal Silicon Nitride layer (SiN-2) is then deposited (Figure 4.3c). The Nitride deposited on the sidewalls of Poly-2 in this step will form the dielectric transducers for the devices. The sacrifical oxide OX-1 and the Nitride SiN-2 are then patterned to allow electrical contact to Poly-0 for routing and anchors (Figure 4.3d). This is followed by the deposition of a thick $2 \mu m$ conformal layer of Polysilicon (Poly-2) (Figure 4.3e). As in the case of the Cornell-based fabrication process, this Polysilicon layer must be planarized with Chemical Mechanical Polishing, with an etch stop on the hard oxide mask OX-2. The Poly-2 layer is then patterned to define the final resonator shape (Figure 4.3f). The sacrificial oxide layer OX-1 is etched away in Hydrofluoric Acid to release the resonators, followed by a Critical Point Dry to prevent stiction (Figure 4.3g). A final metallization step defines probe pads and routing to the device. Figure 4.4 shows an SEM of a $4.5 \mathrm{GHz}$ all-Polysilicon 


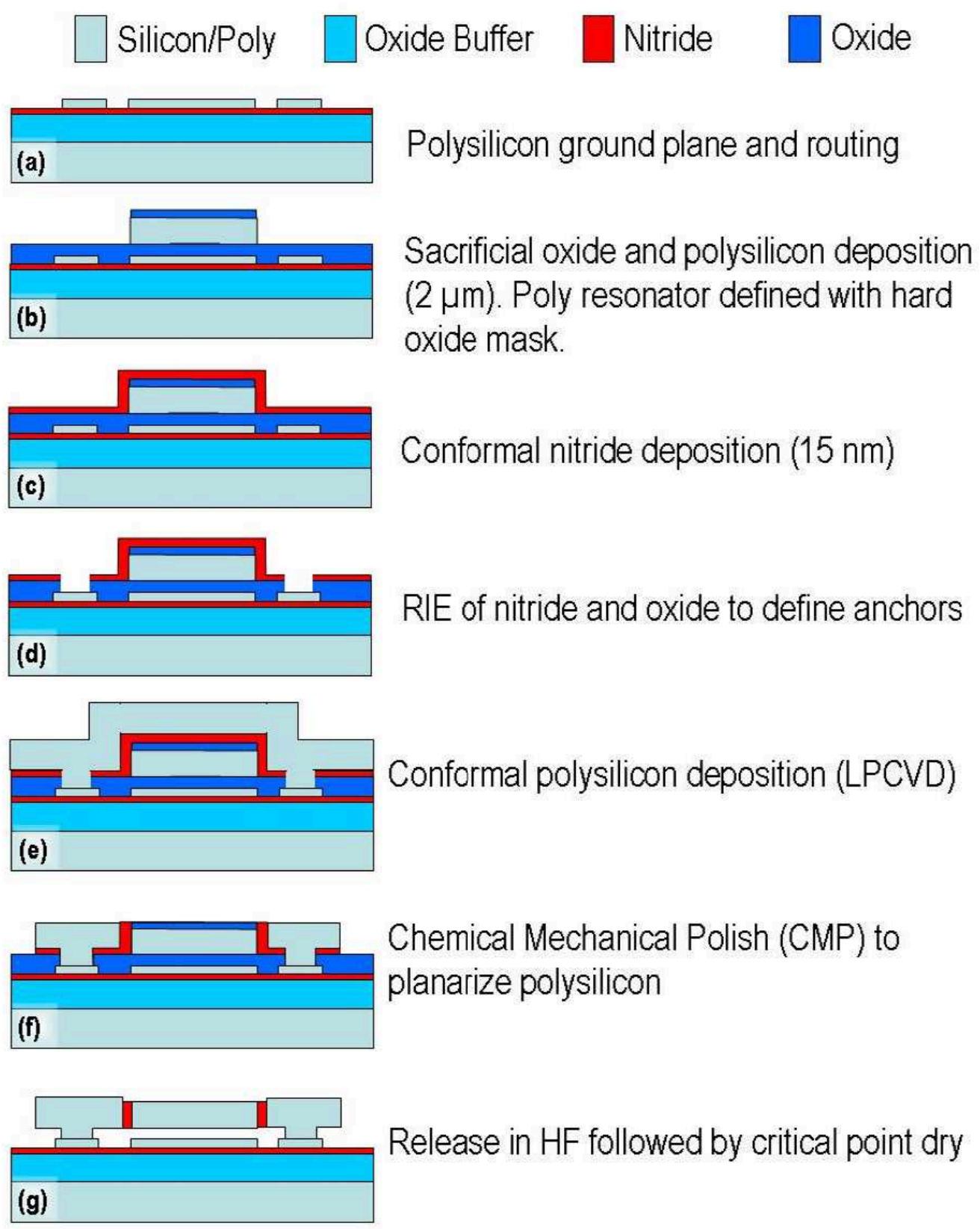

Figure 4.3: An abbreviated illustration of the Sony-based surface micromachining process for dielectrically transduced resonators. The longitudinal resonators are comprised of Polysilicon with $15 \mathrm{~nm}$ Silicon Nitride transducers. 


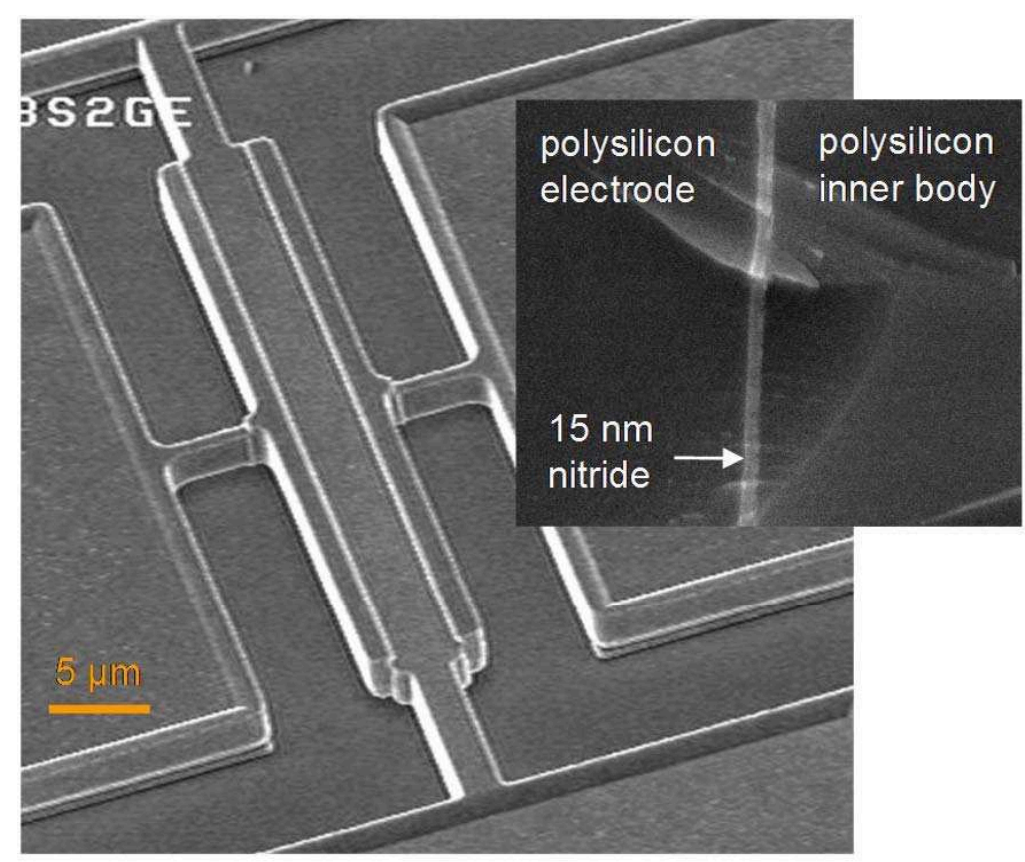

Figure 4.4: Left: Scanning Electron Micrograph of an all-Polysilicon dielectrically transduced silicon bar resonator $(8.5 \mu \mathrm{m}$ long $\times 40 \mu \mathrm{m}$ wide $\times 2 \mu \mathrm{m}$ tall). The inset image shows the $15 \mathrm{~nm}$ Nitride transducer sandwiched between two Polysilicon regions of the resonator.

bar resonator. A cross-sectional SEM of the dielectric shows non-porous film quality. 


\section{CHAPTER 5}

\section{INTERNAL DIELECTRIC TRANSDUCTION: MEASURED RESULTS}

\subsection{Capacitive Drive And Sense}

Capacitive electromechanical resonators can be used as passive mixers due to their nonlinear electrostatic actuation. The resonator can thus be characterized by measuring the conversion loss of the mixer. A scalar mixing measurement (Figure 5.1) similar to [32] using an Agilent PNA was performed to obtain the frequency response of the resonator. A scalar mixer calibration technique traditionally used to characterize RF mixers was implemented to measure the performance of the high frequency resonators. This method circumvents capacitive losses and parasitic transmission-line resonances in the probe-pads and routing of the 3-port MEMS device and provides an accurate measurement of mechanical $Q$ at frequencies well above $1 \mathrm{GHz}$.

Standard 2-port measurements for which the input and measured frequencies are the same require de-embedding structures (namely, short, open, and through structures) to cancel parasitic capacitance, inductance, and resistance from the device measurement. In 3-port scalar mixing measurements, however, parasitic feedthrough currents occur off the resonant (measured) frequency. Since no de-embedding is performed in this measurement, there exists a parasitic resistance from the probe tips to and from the device. The resistance decreases the total signal strength, resulting in an uncalibrated absolute performance of the device. The absolute $R_{X}$ of the resonator cannot be extracted from the measurement without resistive de-embedding, and will appear higher than expected. However, for the purposes of this experiment, the absolute per- 
formance of the device is not critical. Rather, the relative performance of the resonator operating at two different frequencies is investigated. Since the same device is probed for both harmonics and the probe tips remain fixed for the entire measurement, the resistive path from the probe tip to the device remains constant. A comparison of resonance at both harmonics therefore provides useful information about the relative transduction efficiency of the two resonant modes.

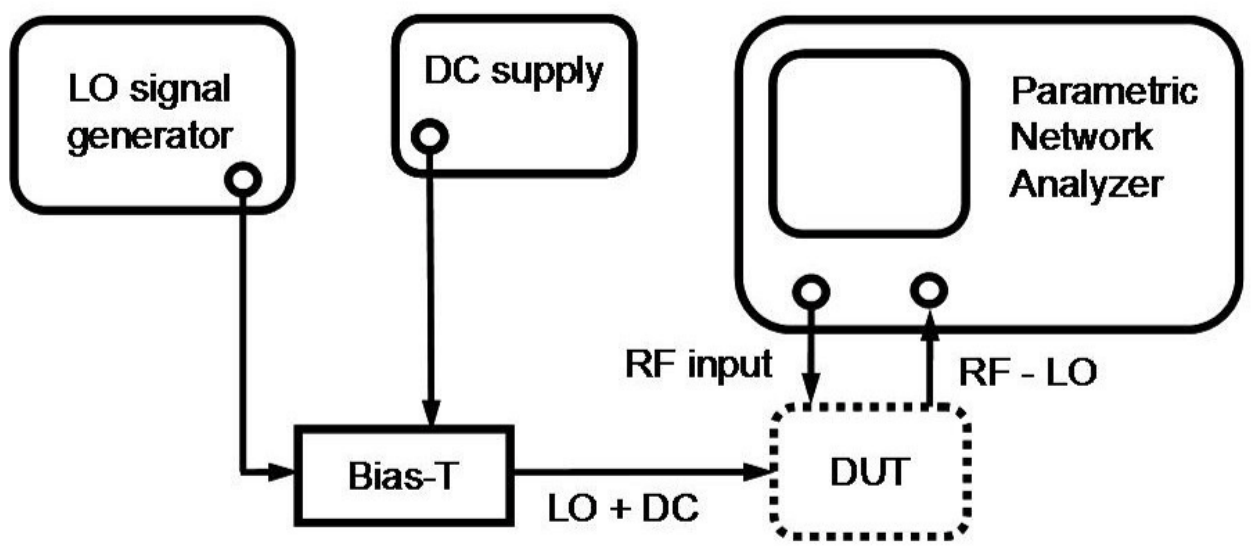

Figure 5.1: Schematic of scalar mixer measurement of the 3-port MEMS resonator. The resonator (DUT) acts as a mixer for the input RF and $\mathrm{LO}$ signals. The resonance is detected at RF - LO, thus preventing effects of feed-through capacitance in the transmitted frequency response

Devices were tested at room temperature in a Lakeshore vacuum probe station, applying a $5 \mathrm{~V}$ bias, $-10 \mathrm{dBm} \mathrm{LO}$, and $0 \mathrm{dBm}$ RF input. The resonators frequency response is presented in Figure 5.2. The LO leakage of the device 
(grey traces) was obtained by setting the bias voltage to $0 \mathrm{~V}$. Acoustic resonance was excited when a $5 \mathrm{~V}$ bias was applied (black traces). The 9th harmonic, with a $Q$ of 11,200 , shows a $9.8 \mathrm{~dB}$ signal improvement over the 3 rd harmonic, with a $Q$ of only 1,700 . The $4.51 \mathrm{GHz}$ resonance has an $f . Q$ product of $5.1 \times 10^{13}$.

The motional impedance in Equation 3.7 is inversely proportional to $Q$. To extract the relationship of transducer efficiency with frequency scaling, we normalize the scalar conversion loss at resonance by the $Q$ of the harmonic. With this normalization, we can directly compare the performance of the two harmonics. Taking this into account, the $4.51 \mathrm{GHz}$ normalized signal improves by 2 $\mathrm{dB}$ relative to the $1.53 \mathrm{GHz}$ normalized response. The analytical model predicts a $3 \times$ improvement in motional impedance between the 3rd and 9th harmonics, translating to a $4.7 \mathrm{~dB}$ signal improvement. The discrepancy may be due to small misalignment $(<200 \mathrm{~nm}$ ) and the width-distortion of the longitudinal mode-shape described above. In particular, the transducer efficiency is more sensitive to misalignment of the dielectric film position at higher harmonics, since the misalignment corresponds to a larger fraction of the total wavelength at higher frequencies. Therefore, a misalignment in the device under test degrades the performance of the 9th harmonic more than the 3rd harmonic, contributing to a smaller relative transduction enhancement than expected.

The high-yield fabrication process performed at Sony (Figure 4.3) allowed for testing across multiple resonators. The measured motional impedance of the 3rd and 9th harmonics of 8 Sony-based resonators with varying dielectric position is shown in Figure 5.3. Since the dielectric transducers are incorporated into the resonant mode, they can be placed anywhere in the resonator body. As dictated in Equation 3.7, $R_{X}$ follows an inverse $\cos ^{2}$ behavior with dielectric position along the bar, and demonstrates minima when the dielectric is placed at 

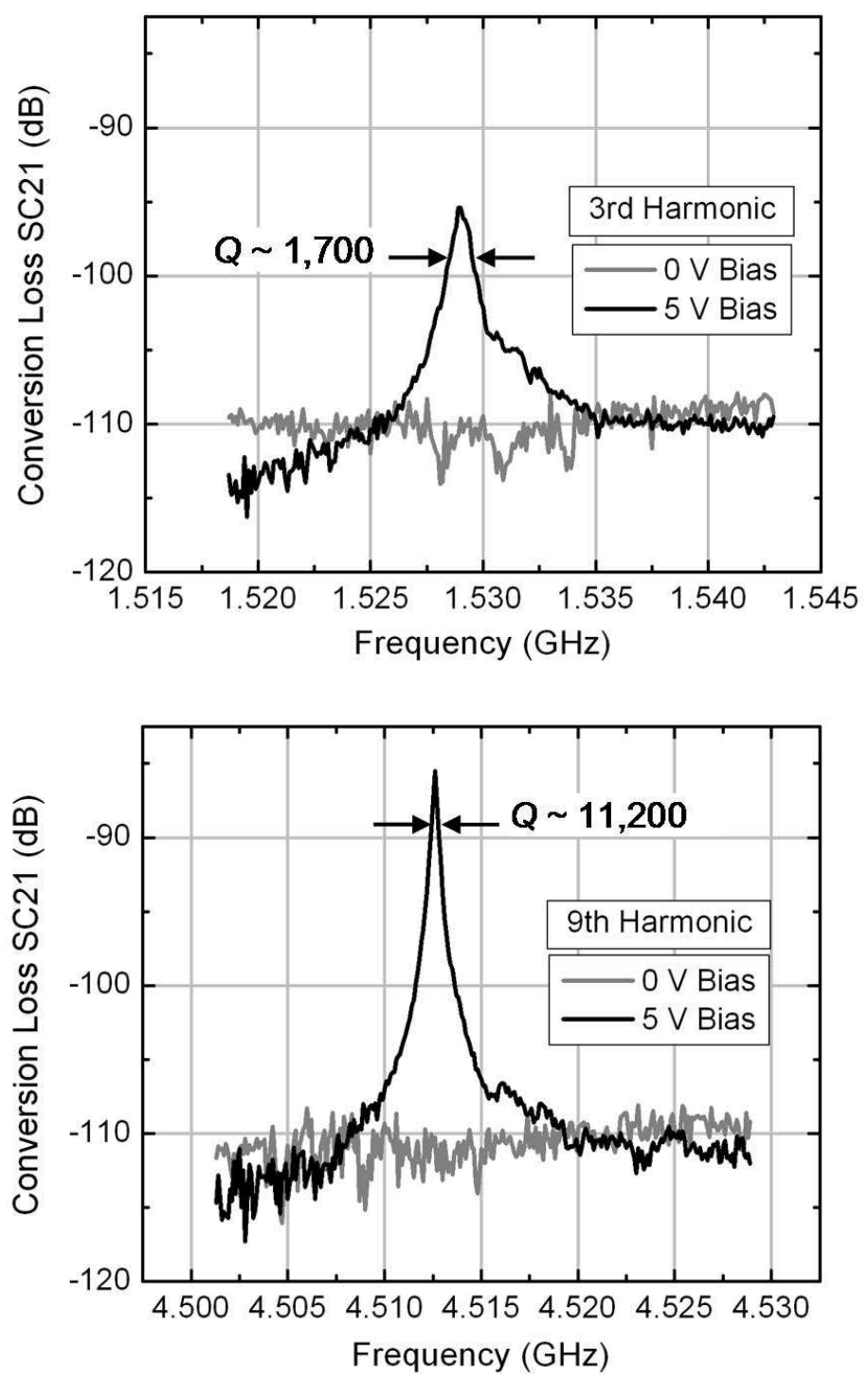

Figure 5.2: Measured frequency response of 3rd and 9th harmonic resonance of the silicon bar resonator. The grey traces $(0 \mathrm{~V})$ indicate the LO leakage of the device. Applying a $5 \mathrm{~V}$ bias excites acoustic resonance, shown in black. The 9th harmonic exhibits a $9.8 \mathrm{~dB}$ absolute improvement in signal strength over the 3rd harmonic mode, with a $Q$ increase of $6.6 \times$. Normalizing the resonant peaks by $Q$, we extract a $2 \mathrm{~dB}$ enhancement of transduction efficiency in the $4.5 \mathrm{GHz}$ resonance relative to the 1.5 $\mathrm{GHz}$ resonant mode. 
a displacement node. Measuring the 3rd and 9th harmonics of longitudinal resonance across 8 different resonators with identical overall dimensions, we can map the $R_{X}$ dependence on dielectric position. As seen in Figure 5.3, as the dielectric approaches the center of the resonator, transduction efficiency of the 3rd harmonic diminishes to the point where a signal cannot be detected. Meanwhile, the 9th harmonic follows a second minimum in $R_{X}$ towards the center of the bar. With the dielectric at position \# 1, the transduction efficiency for the 9th harmonic is much higher than that of the 3rd, providing selective 9th harmonic excitation. The $R_{X}$ asymptote for the 9th harmonic at position \# 2 enables 3rd harmonic excitation with no resonance at the 9th. Finally, the dielectric can be placed at displacement nodes common to both harmonics, as shown at position \# 3. In this case, both 3rd and 9th harmonics are excited at maximum efficiency. This demonstration of selective mode excitation provides flexibility of design in IDT resonators.

In addition to the study of dielectric position, frequency scaling trends extracted from multiple resonators with optimal dielectric positioning are shown in Figures 5.4 and 5.5. The frequency response of 3rd and 9th harmonics of two resonators provides frequency scaling information from $1.55 \mathrm{GHz}$ to $6.20 \mathrm{GHz}$. The resonant modes across multiple resonators exhibit high $f . Q$ products characteristic of IDT resonators. The 3rd harmonic resonance $4.7 \mathrm{GHz}$ exhibits an $f . Q$ product of $3.1 \times 10^{13}$, the highest $f . Q$ product in Polysilicon reported to date. The 9th harmonic at $6.2 \mathrm{GHz}$ is the record high acoustic resonance in silicon to date. The motional impedance of Equation 3.7 is a function of both harmonic nand quality factor $Q$. To directly compare resonance with varying harmonics and $Q, R_{X}$ must be normalized as shown in Figure 5.5. The nominal $Q_{\text {avg }}=5065$ for normalization is chosen as the average $Q$ of all four resonant peaks under 
comparison. In accordance with Equation 3.7, the measured internal dielectric transduction efficiency improves with increasing frequency. This result verifies the ability of internal dielectric transduced resonators to scale favorably to even higher frequencies.
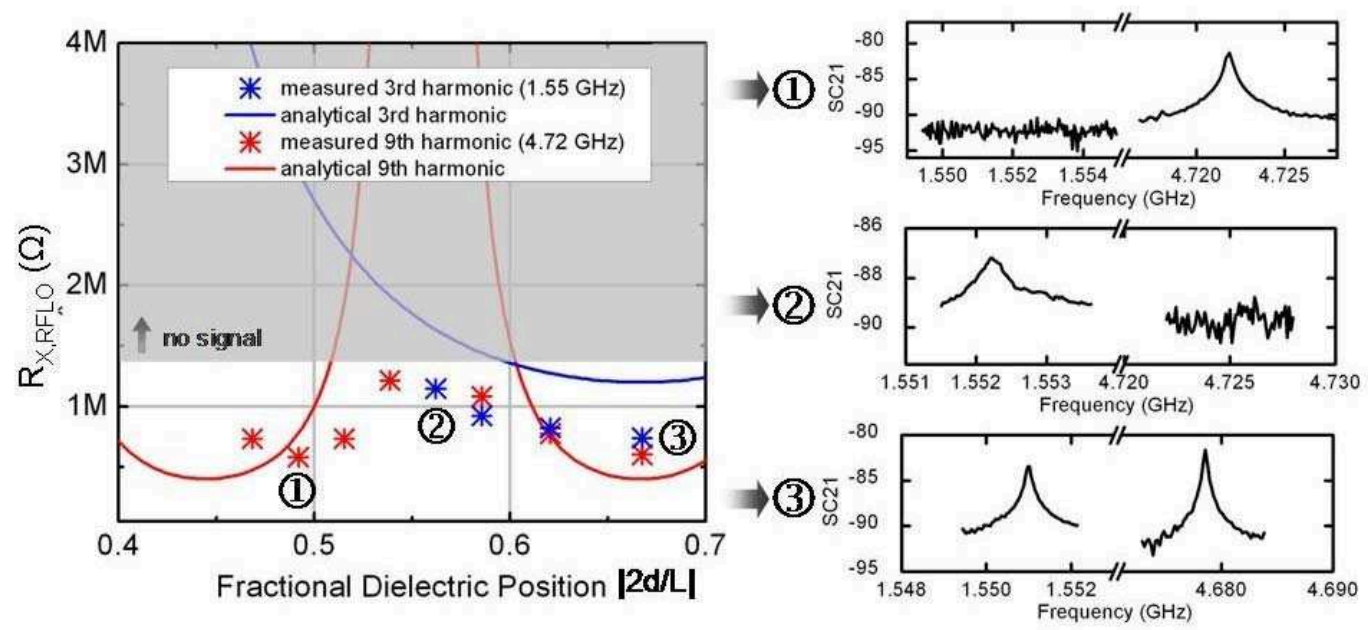

Figure 5.3: Motional impedance vs. position of dielectric transducer in 3rd and 9 th harmonics of 8 different resonators. Selective positioning of dielectric can excite \# 1 only 9th harmonic at $4.72 \mathrm{GHz}$, \# 2 only 3rd harmonic at $1.55 \mathrm{GHz}$, and \# 3 both 3rd and 9th harmonics.

This experiment demonstrated selective excitation of targeted harmonics in Polysilicon dielectrically transduced bar resonators by varying dielectric position in the resonator body. It also verified the predicted IDT frequency scaling behavior, with improved transduction efficiency at higher frequency. RF resonators up to $6.2 \mathrm{GHz}$ were demonstrated, with $f . Q$ products up to $3.1 \times 10^{13}$, 


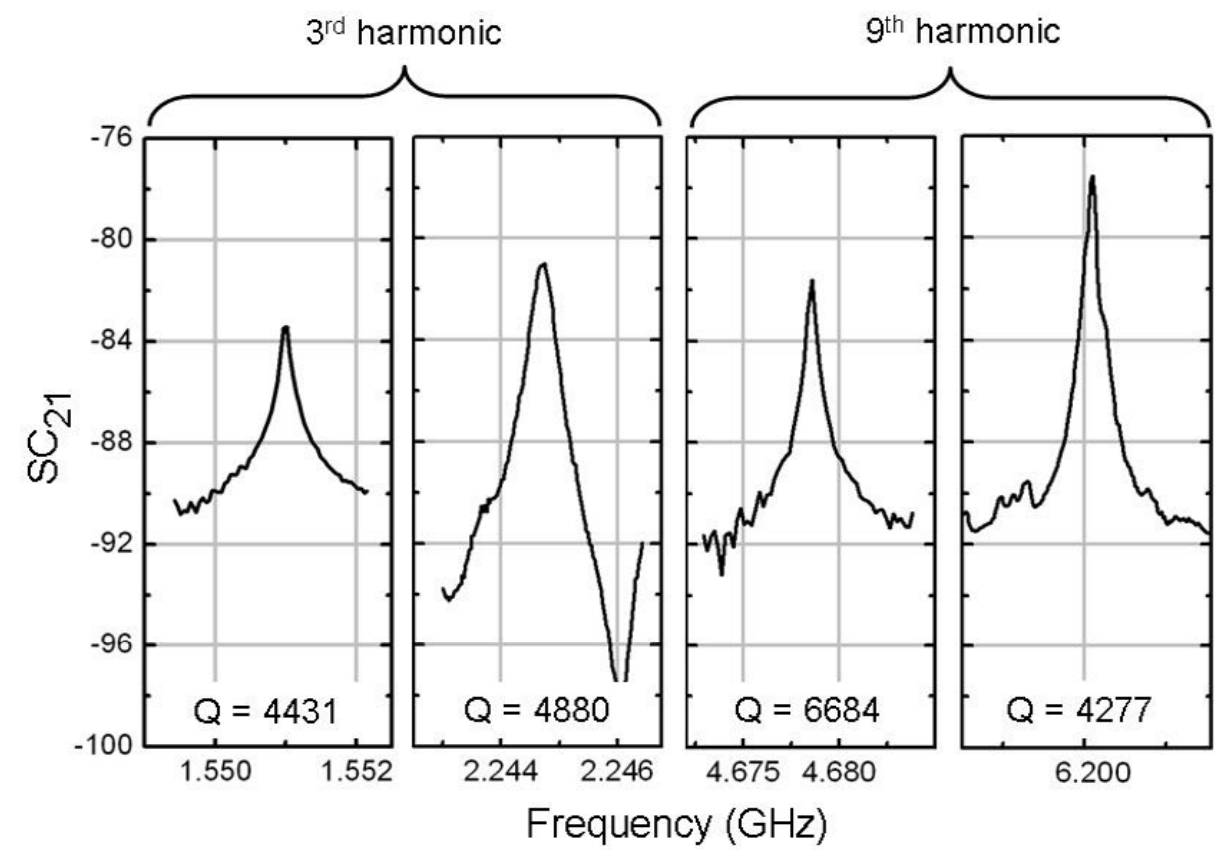

Figure 5.4: Measured frequency response of 3rd and 9th harmonics of two bar resonators. The 9 th harmonic at $6.2 \mathrm{GHz}$ marks the highest frequency measured in silicon to date.

the highest reported to date in Polysilicon. The high quality factors measured in these IDT resonators indicates the ability to scale resonators to high frequency without compromising $f \cdot Q$ product.

\subsection{Capacitive Drive And Piezoresistive Sense}

Capacitive sensing at $\mathrm{GHz}$ frequencies is challenging due to large nominal and feed-through capacitance intrinsic to the measurement. Three-port scalar-mixer measurements are often required. To overcome this obstacle, piezoresistive 


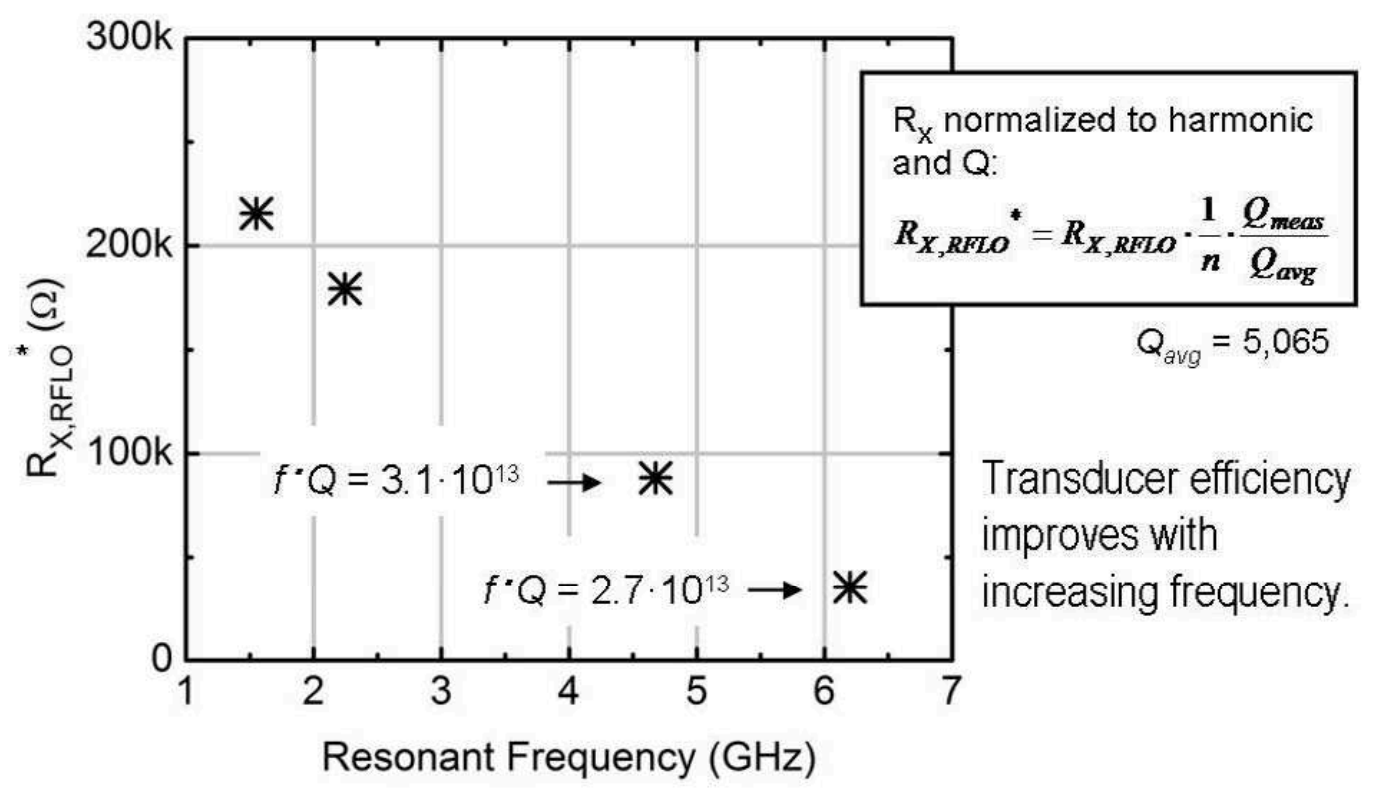

Figure 5.5: Motional impedance scaling with resonant frequency. Comparison of resonators with varying harmonics $\mathrm{n}$ and quality factors (Q) necessitates normalization of $\mathrm{Rx}$ as shown (normalization factor $Q_{0}=5000$ ). As predicted by theory, Rx improves with increasing frequency.

sensing of a capacitively actuated resonator is implemented [33]. There are several benefits to piezoresistive detection. Geometric and frequency scaling are considerably more favorable than in the case of capacitive sensing. Additionally, independent control of the drain current enables us to set the piezoresistive transconductance gm to be as large as possible. When a drain current Id flows through a mechanically resonating structure, the piezoresistive change in the resistance $d R / R$ generates a transconductance

$$
g_{m}=\frac{d R}{R} \frac{I_{d}}{v_{i n}}
$$


where $v_{\text {in }}$ is the $\mathrm{AC}$ voltage capacitively exciting resonance across the dielectric films. The change in the resistance due to piezoresistivity follows

$$
\frac{d R}{R}=\pi_{t} \sigma_{t}+\pi_{l} \sigma_{l}
$$

where $\pi_{t}$ and $\pi_{l}$ are the transverse and longitudinal piezoresistive coefficients, and $\sigma_{t}$ and $\sigma_{l}$ are the transverse and longitudinal stresses, respectively. The stress is defined by the resonant mode shape with amplitude of vibrations given by Equation 3.4. Averaging the contribution of Equation 5.1 over all points in the resonator, weighting each point by the ratio of total current density at that point to the total current density, the total fractional piezoresistive change is given by

$$
\frac{d R}{R}=\frac{\sum_{i} j_{i}\left(\pi_{t i} \sigma_{t i}+\pi_{l i} \sigma_{l i}\right)}{\sum_{i} j_{i}}
$$

In the case of dielectrically actuated longitudinal-mode resonator, acoustic waves travel perpendicular to the drain current density, shown in Fig. 8. Fig. 9 shows the analytical results of the contribution to the piezoresistive signal distributed over the resonator body, as defined by equation 10 . The symmetry of the resonant mode and uniformity of the current flow result in a cancellation of most of the signal. With this geometry, the primary contribution to the output signal comes from vibrations in and near the suspension beams.

The 9th harmonic longitudinal resonance of the bar at $4.5 \mathrm{GHz}$ was used for piezoresistive measurements. The resonator was tested at room temperature in a vacuum probe station. A schematic of the experimental setup is shown in Figure 5.8. A short-open-load-through (SOLT) calibration on a ceramic substrate 

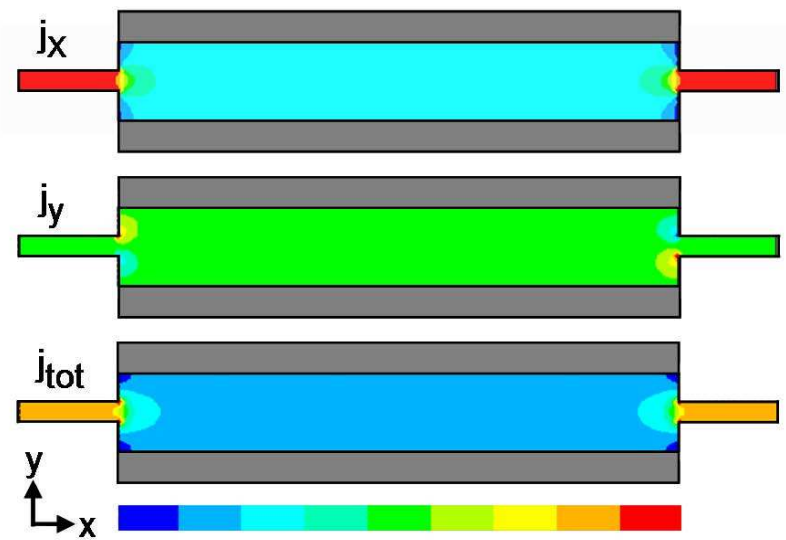

Figure 5.6: Contour plot of current density in the resonator. Current flow is uniform through most of the resonator body. Current nonuniformity at input and output of the resonator is the primary source of piezoresistive signal.

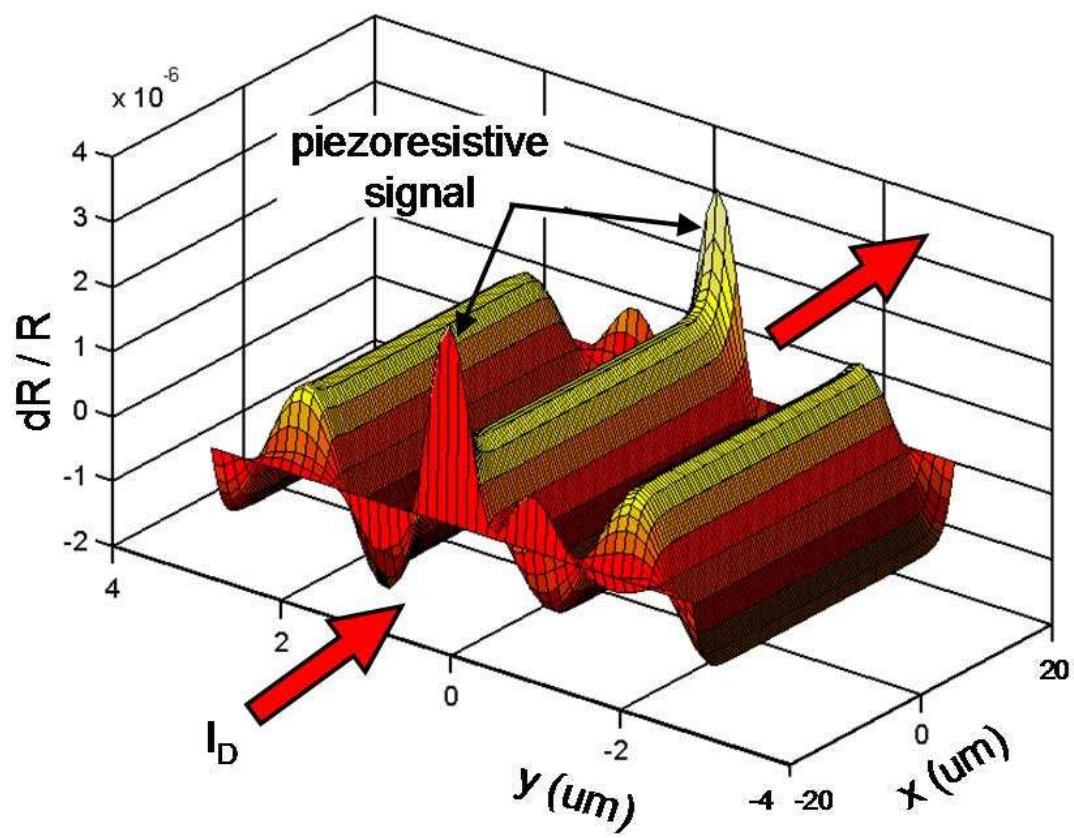

Figure 5.7: Spatial distribution of piezoresistive signal. The symmetry of the strain in the resonator results in cancellation of the signal in the majority of the body. The piezoresistance peaks near the routing beams generate the detected resonant signal. 
was first performed to remove parasitics up to the probe tips, followed by deembedding using short, open, and through structures on-chip. Both ends of the resonator were biased to a $V_{G}$ of $10 \mathrm{~V}$, and a $0 \mathrm{dBm}(0.2 \mathrm{~V}) \mathrm{AC}$ excitation from an Agilent parametric network analyzer (PNA) was superimposed to capacitively generate resonance. A drain current across the resonator, defined by a bias voltage $V_{d}$, was modulated piezoresistively and detected by the PNA. The transconductance is extracted from the admittance parameters by

$$
g_{m}=Y_{21}-Y_{12}
$$

This technique is identical to microwave frequency measurement of the transconductance in transistors.

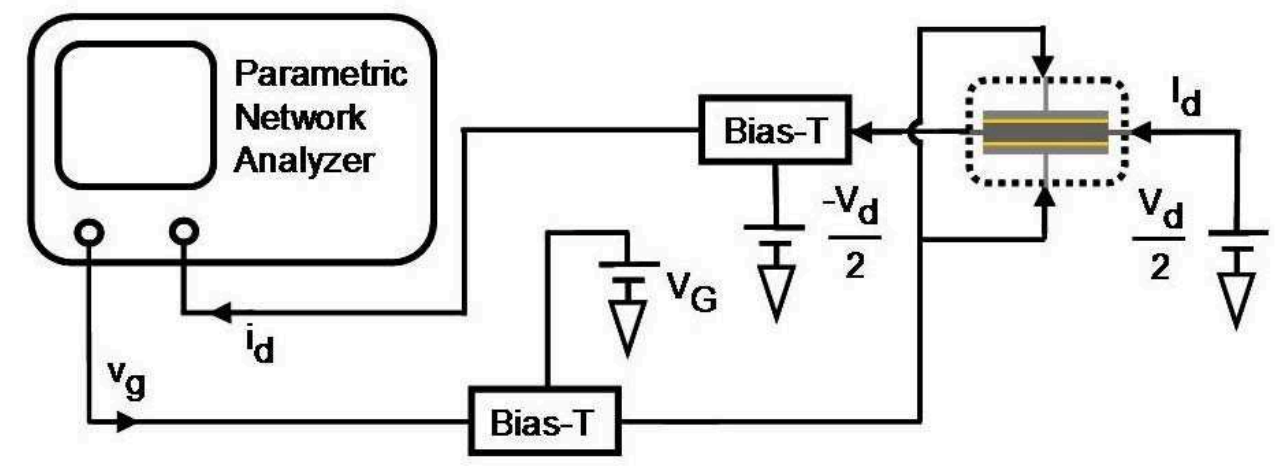

Figure 5.8: Schematic of measurement setup for internal dielectric actuation and piezoresistive detection. 
The measured frequency response of the 9th harmonic longitudinal mode with drain currents of $1.22,11.82$, and $23 \mu A$ is presented in Figure 5.9. At 23 $\mu A$, a resonant frequency of $4.41 \mathrm{GHz}$ with an electromechanical $Q$ of 8180 is observed, with a piezoresistive transconductance of $1.1 \mu \mathrm{A} / \mathrm{V}$. The power dissipated in the resonator is $0.46 \mathrm{~mW}$.

Several trends occur as the drain current through the resonator increases. As drain current increases, resonant frequency decreases, as shown in Figures 5.9 and 5.10. This is due to thermal expansion of the silicon bar and Young's modulus tuning as a result of joule heating. A $1.6 \%$ tuning is observed varying the current flow through the resonator from $1.22 \mu \mathrm{A}$ to $23 \mu \mathrm{A}$.

Figure 5.11 presents the experimental shift in electromechanical $Q$ as the current increases. This steady-state trend is obtained after cycling the current through the resonator from low to high multiple times, allowing for desorption of molecules at the surface of the resonator through heating. At a maximum current of $23 \mu \mathrm{A}$, the resonant signal exhibits an electromechanical $f \cdot Q$ product of $3.6 \times 10^{13}$. While the three-port scalar mixer measurement used for capacitive sensing provides an accurate measure of the resonators mechanical $Q$, the quality factor obtained in the two-port piezoresistive measurement combines the mechanical and electrical contributions to $Q$, resulting in an electromechanical $Q$ commonly quoted in literature. This electromechanical $f . Q$ product is the highest measured to date in silicon. As seen in Figure 5.12, piezoresistive transconductance scales linearly with drain current, dictated by equation 8 , with a $1.1 \mu S$ signal at $23 \mu \mathrm{A}$ of drain current. The extracted piezoresistive $\mathrm{dR} / \mathrm{R}$ for this device is $1 \%$.

The concept of internal dielectric transduction was proposed and experimentally verified. A $4.5 \mathrm{GHz}$ longitudinal bar resonator is demonstrated, mark- 

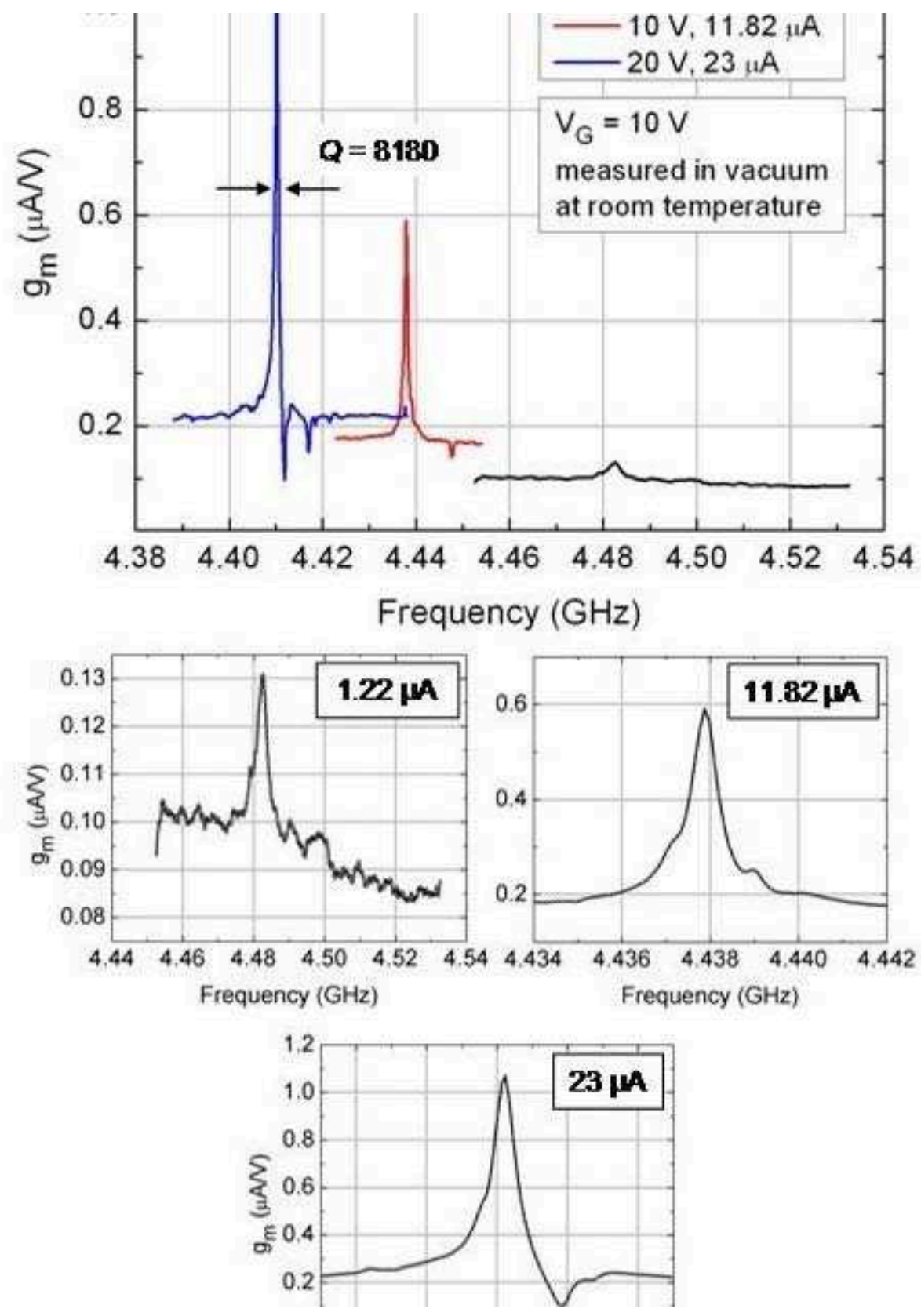

Figure 5.9: Measured frequency response of transconductance gm of the resonator for varying drain currents. 


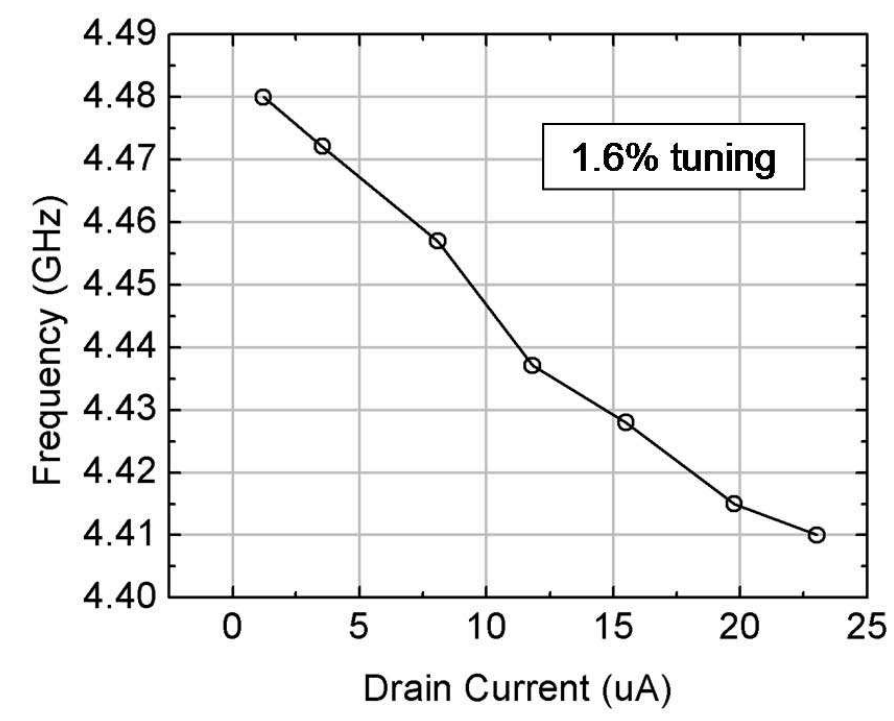

Figure 5.10: Measured resonant frequency scaling with increasing drain current. Resistive heating thermally expands the resonator, decreasing resonant frequency with increasing internal temperature

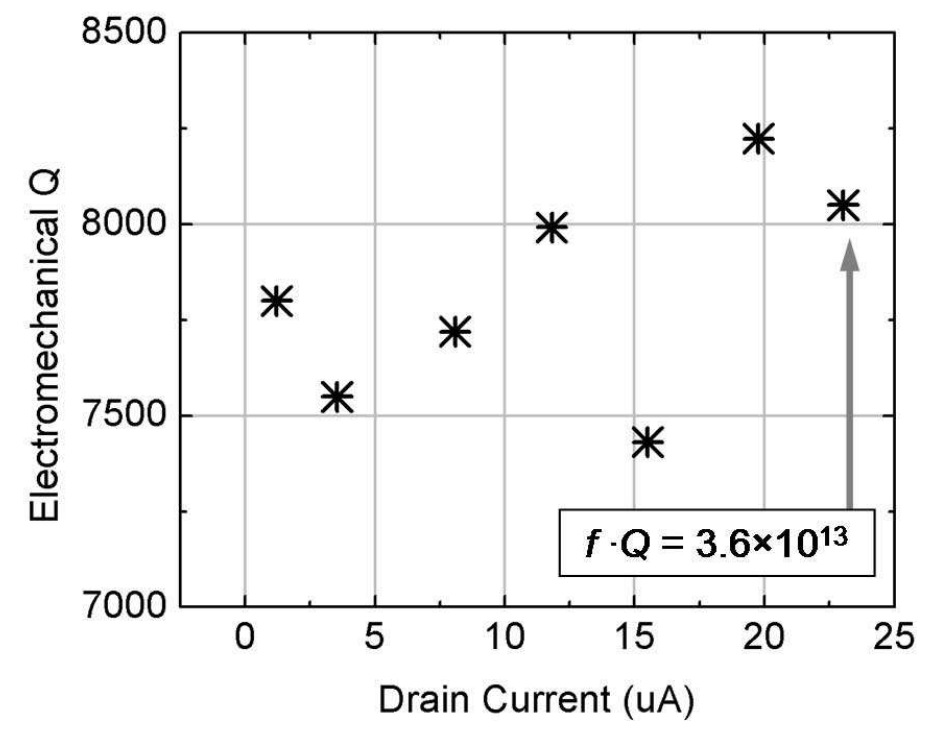

Figure 5.11: Measured electromechanical $Q_{e m}$ with increasing drain current. 


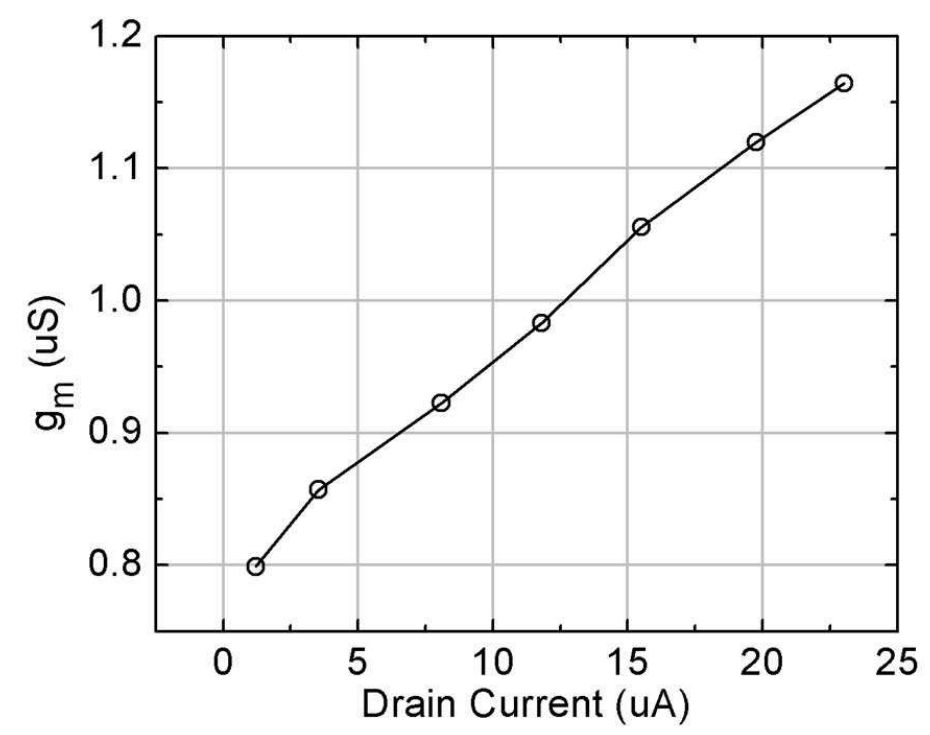

Figure 5.12: Experimental resonator transconductance $g_{m}$ scaling with increasing drain current.

ing the highest frequency measured to date in silicon. The 3rd and 9th harmonics of longitudinal vibration were excited in a silicon bar resonator, demonstrating a $9.8 \mathrm{~dB}$ absolute improvement in signal strength and $2 \mathrm{~dB}$ ( $Q$-normalized) enhancement in transduction efficiency for the 9th harmonic $(4.5 \mathrm{GHz})$ relative to the $3 \mathrm{rd}$ harmonic $(1.5 \mathrm{GHz})$. The $4.5 \mathrm{GHz}$ resonance exhibits a mechanical $f . Q$ product of $5.1 \times 10^{13}$. These results indicate improved resonator performance with increased frequency, providing a means of scaling MEMS resonators to previously unattainable frequencies in silicon.

Piezoresistive sensing was presented as a viable improvement over internal dielectric capacitive sensing in high frequency resonators, offering a two-port configuration to facilitate direct incorporation of RF MEMS resonators into integrated circuit applications. The measured resonance at $4.41 \mathrm{GHz}$ demonstrates an electromechanical $Q$ of 8180 . The $f . Q$ product of the resonator is higher than previously measured in silicon, indicating the promise of internal dielectric ac- 
tuation in $>1 \mathrm{GHz}$ resonators. Piezoresistive sensing, which does not require a scalar mixer measurement, yields a measurement of the electromechanical $Q$ as opposed to a purely mechanical $Q$. This is the true measurement of $Q$ used in the design of integrated devices. A low power consumption of $<1 \mathrm{~mW}$ and control of gm make these NEMS-Transconductor hybrid resonators ideal candidates for integration into high-frequency CMOS technology. 
CHAPTER 6

THE RESONANT BODY TRANSISTOR: THEORY

\subsection{Motivation}

As we scale to deep sub-micron (DSM) technology, transistor threshold frequencies increase, enabling the design of CMOS circuits for RF and mm-wave applications up to $67 \mathrm{GHz}$. However, such high-frequency CMOS transistors have very limited gain, resulting in poor output power. A successful transition of these applications into DSM CMOS technology therefore requires high-Q, low-power components operating at high frequencies. Another challenge facing DSM circuits is the increasing density of devices, projected to reach $10^{11}$ devices $/ \mathrm{cm}^{2}$. At such densities, clocking networks and the power consumption associated with them necessitate implementation of low-power local clocks with the potential for global synchronization.

In 1967, Nathanson et al. demonstrated the Resonant Gate Transistor (RGT) [34], driving resonance in a conductive cantilever with an air-gap capacitive electrode. The RGT cantilever functions as the gate of an air-gap transistor, with output drain current modulated by the cantilever resonant motion. This chapter introduces the Resonant Body Transistor (RBT), a Nano Electromechanical (NEM) resonator integrating a sense transistor directly into the resonator body, geometrically similar to a suspended split-gate FinFET, as shown in Figure 6.1. Combining the benefits of FET sensing with the frequency scaling and high- $Q$ capabilities of IDT bar resonators, the Resonant Body Transistor (RBT) can be integrated into a standard CMOS process for low power clock generation and high- $Q$ tank circuits. 


\subsection{Principle Of Operation}
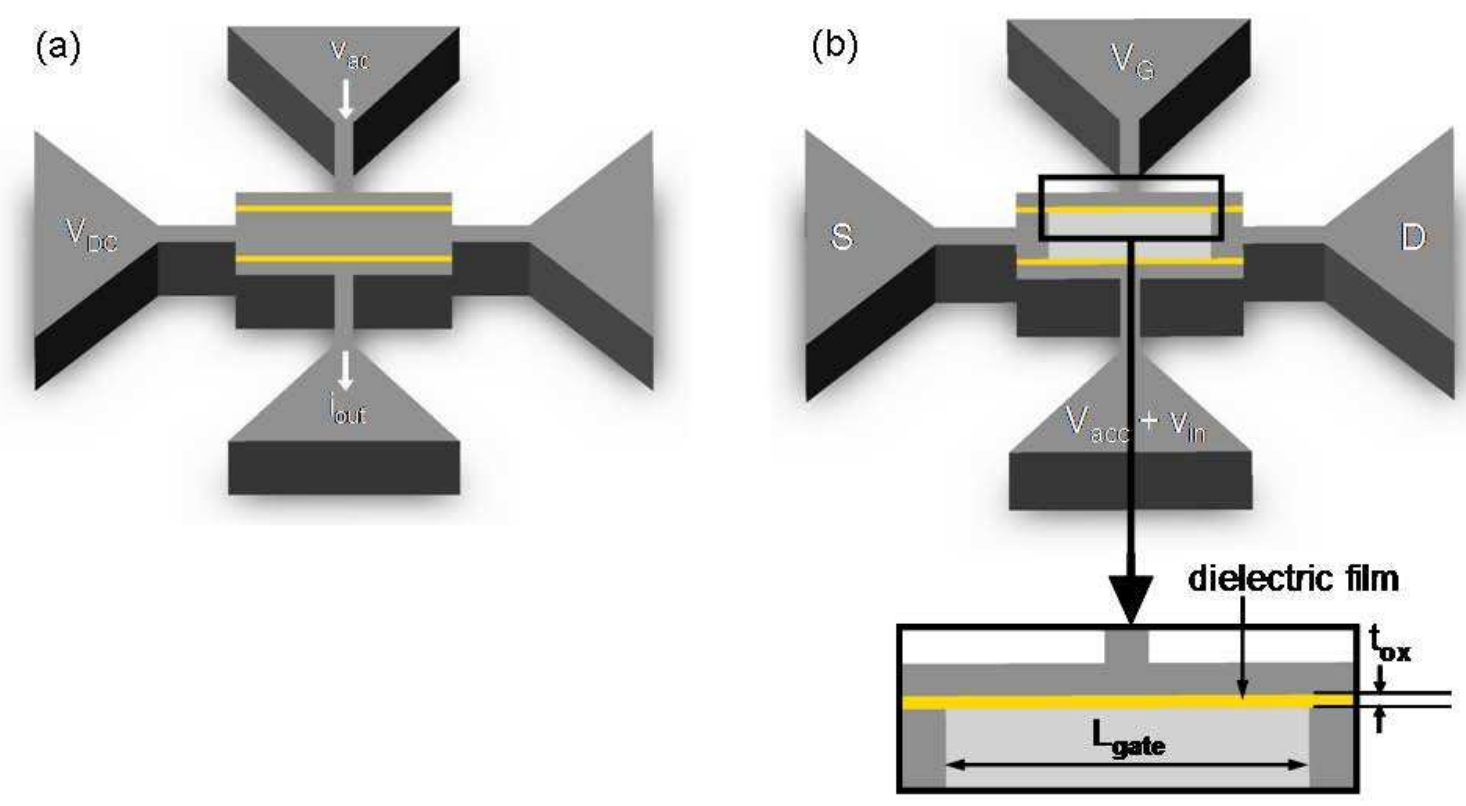

Figure 6.1: (left) Longitudinal bar resonator driven and sensed using Internal Dielectric Transduction. (right) A Resonant Body Transistor (RBT) driven with Internal Dielectric Transduction.

The RBT (Figure 6.1) is geometrically similar to the longitudinal-mode internal dielectrically transduced resonator, apart from the doping in the center of the resonator which allows for accumulation and inversion layers in the resonator body. For the NMOS RBT under consideration, the center region is undoped. The RBT is biased as shown in Figure 6.2. A gate voltage VG (limited by the breakdown voltage of the dielectric) is applied to one gate, generating an inversion layer. The source is tied to ground, while the drain is biased to 
Table 6.1: Symbol Definition

\begin{tabular}{|l|l|}
\hline \hline$L$ & length of resonator \\
\hline$W$ & width of resonator \\
\hline$h$ & height of resonator \\
\hline$L_{g}$ & gate length \\
\hline$d$ & distance of dielectric from center of bar \\
\hline$g$ & dielectric thickness \\
\hline$n$ & order of harmonic \\
\hline$k_{n}$ & wavenumber $=n \pi / L$ \\
\hline$Y$ & Youngs modulus \\
\hline$\rho$ & mass density \\
\hline$\varepsilon_{f}$ & permittivity of dielectric \\
\hline$\mu_{n}$ & electron mobility \\
\hline$p i_{110}$ & tangential piezoresistive coeff. (110) \\
\hline$Q$ & quality factor of resonance \\
\hline$U_{0}$ & amplitude of vibrations at resonance \\
\hline$x$ & position along the bar (x $=0$ at center) \\
\hline$V_{D C}$ & bias voltage \\
\hline$V_{D}$ & drain voltage \\
\hline$V_{G}$ & gate voltage \\
\hline$V_{a c c}$ & voltage at accumulation gate \\
\hline$V_{T}$ & threshold voltage \\
\hline$I_{D}$ & drain current \\
\hline$v_{a c}$ & ac input voltage \\
\hline$i_{o u t}$ & ac output current \\
\hline
\end{tabular}


Table 6.2: Assumed Constants

\begin{tabular}{|l|l|}
\hline \hline$\varepsilon_{0}$ & $8.85 \times 10^{-12} \mathrm{~m}^{-3} \mathrm{~kg}^{-1} \mathrm{~s}^{4} \mathrm{~A}^{2}$ \\
\hline$\varepsilon_{f}$ & $7 \varepsilon_{0}$ \\
\hline$h$ & $250 \mathrm{~nm}$ \\
\hline$W$ & $800 \mathrm{~nm}$ \\
\hline$d$ & displacement node \\
\hline$g$ & $10 \mathrm{~nm}$ \\
\hline$n$ & 3 \\
\hline$Y$ & $170 \times 10^{9} \mathrm{~Pa}^{-3}$ \\
\hline$\rho$ & $2330 \mathrm{kgm}^{-3}$ \\
\hline$\mu_{n}$ & $300 \times 10^{-4} \mathrm{Vm}^{-2} \mathrm{~s}^{-1}$ \\
\hline$\pi_{110}$ & $17 \times 10^{-11} \mathrm{~Pa}^{-1}$ \\
\hline$Q$ & $5 \times 10^{13} / f$ \\
\hline$V_{D C}$ & $3 \mathrm{~V}$ \\
\hline$v_{a c}$ & $0.1 \mathrm{~V}$ \\
\hline$V_{T}$ & $0.6 \mathrm{~V}$ \\
\hline$V_{G}$ & $3 \mathrm{~V}$ \\
\hline$V_{a c c}$ & $V D-V$ \\
\hline$V_{D}$ & $(V G-V T)+0.1 V$ \\
\hline & \\
\hline
\end{tabular}

$V_{D}>V_{G} V_{T}$ to drive the transistor in saturation. The DC saturation drain current is given by

$$
I_{D}=\frac{1}{2} \mu_{n} \frac{\varepsilon_{f}}{g} \frac{h}{L_{g}}\left(V_{G}-V_{T}\right)^{2}
$$

An accumulation voltage $V_{a c c}$ with an AC excitation voltage $v_{a c}$ is applied 


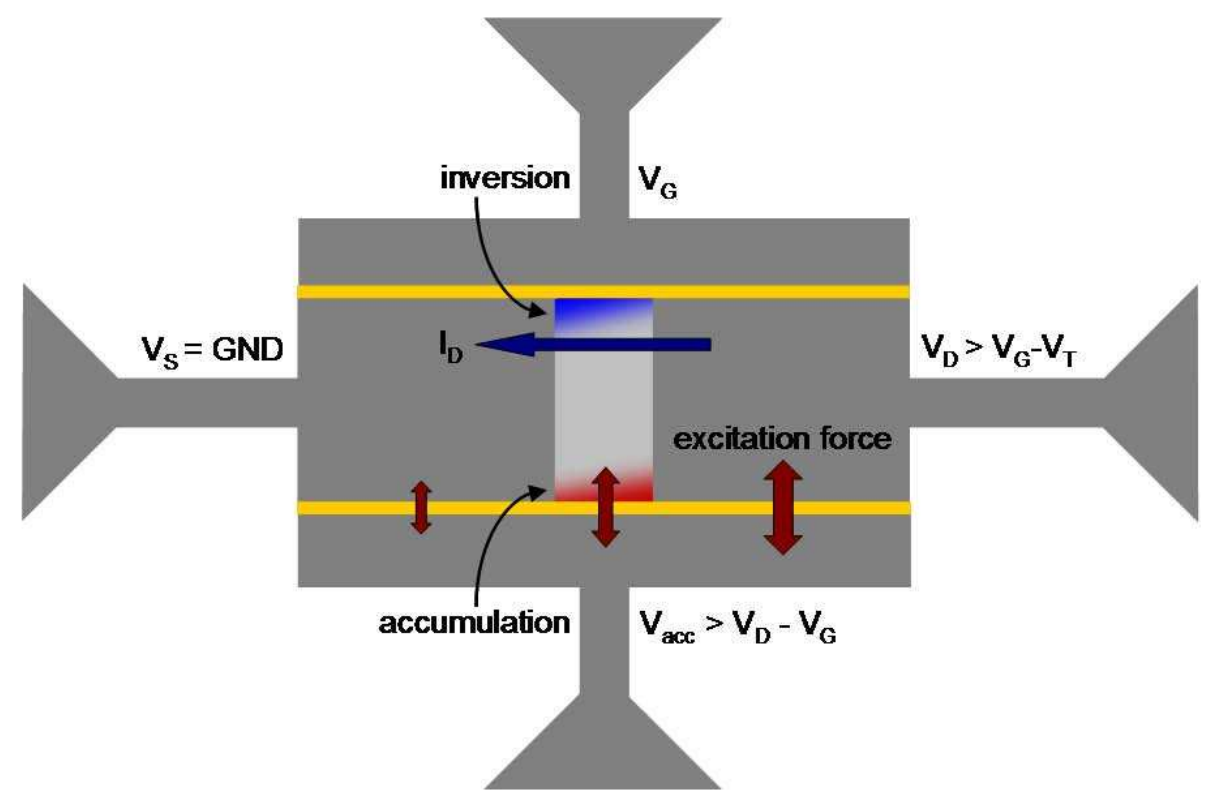

Figure 6.2: Principle of operation for an internal dielectrically driven RBT.

at one gate, driving resonance. $V_{\text {acc }}$ is limited by the breakdown voltage across the dielectric, so that $V_{a c c}>V_{D} V_{G}$. For a breakdown voltage of $3 \mathrm{~V}, V_{G}=3 V$, $V_{D}>2.4 \mathrm{~V}=2.5 \mathrm{~V}, V_{a c c}=-0.5 \mathrm{~V}$ so that the drop from accumulation gate to drain is $3 \mathrm{~V}$. The electrostatic force for actuation is distributed across three regions. The force is strongest between the accumulation gate and the drain region due to the large voltage drop across the dielectric. The force is weakest between the accumulation gate and the source. The amplitude of vibrations of longitudinal resonance is

$\left.U_{0}\right|_{R B T}=\left.U_{0}\right|_{C a p}\left[\frac{1}{V_{D C} W}\left[\left(\frac{W}{2}-\frac{L_{g a t e}}{2}\right)\left(V_{D}-V_{a c c}\right)-\left(\frac{W}{2}-\frac{L_{\text {gate }}}{2}\right) V_{a c c}+\frac{L_{\text {gate }}}{2}\left(V_{D}-V_{a c c}\right)\right]\right]$

where $\left.U_{0}\right|_{\text {Cap }}$ is given in Equation 3.4. The strain induced in the resonator peizoresistively modulates the drain current running through the inversion 
layer. Assuming a piezoresistive coefficient of $\pi_{110}$ for current travelling perpendicular to the normal of elastic wave fronts along (110), the change in mobility is given by

$$
\begin{aligned}
\frac{d \mu_{n}}{\mu_{n}} & =\left.\pi_{110} Y \frac{\partial u}{\partial x}\right|_{\text {inversion }} \\
& =\left.\pi_{110} Y k_{n} U_{0}\right|_{R B T} \cos \left(\frac{k_{n} g}{2}\right)
\end{aligned}
$$

The piezoresistive mobility modulation of Equation 6.3 generates an AC current linearly dependent on the drain current:

$$
\begin{aligned}
\left.i_{\text {out }}\right|_{\text {RBT }} & =I_{D}\left(\frac{d \mu_{n}}{\mu_{n}}+\frac{\left.2 U_{0}\right|_{R B T} \sin \left(\frac{k_{n} g}{2}\right)}{g}\right) \\
& \approx I_{D} \frac{d \mu_{n}}{\mu_{n}}
\end{aligned}
$$

The second term in Equation 6.4 is attributed to change in the gate capacitance as the bar expands and contracts. However, its contribution to current modulation is more than an order of magnitude smaller than that of piezoresistance. The resulting motional impedance is

$$
R_{X, R B T}=\frac{2 n \pi W L_{\text {gate }} g^{3} \csc \left(k_{n} g\right)}{Q h \pi_{110} \mu_{n} \varepsilon_{f}^{2}\left(V_{G}-V_{T}\right)^{2}\left(W\left(2 V_{G}-V_{D}\right)-L_{g a t e}\left(V_{G}-V_{D}\right)\right)}
$$

Figures 6.3 and 6.4 present a comparison of resonator performance for a capacitive IDT resonator and an RBT as a function of increasing frequency. The RBT provides efficient transduction over a wide range of frequencies, with more than an order of magnitude more efficient transduction than the capacitive IDT resonator up to $40 \mathrm{GHz}$. Figure 6.5 shows a zoomed in plot of the RBT motional 
admittance $1 / R_{X}$ scaling with frequency. The motional admittance drops by $3 \%$ from $1 \mathrm{GHz}$ to $60 \mathrm{GHz}$, resulting from geometric limitations of the embedded transistor.

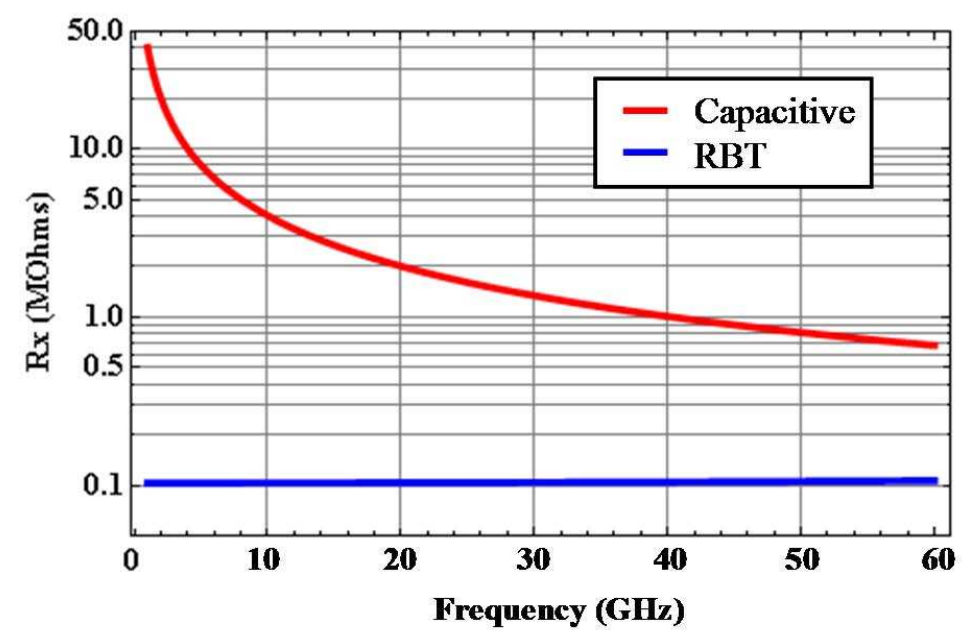

Figure 6.3: Frequency scaling comparison of motional impedance $R_{X}$ for a capacitive resonator and an RBT.

\subsection{Noise Analysis}

\subsubsection{Electrostatic Drive Noise}

Figure 3.1 shows the suspension beams of the resonator used for routing the bias voltage $V_{D C}$ to the resonator body. In practice, there exist additional beams necessary for routing to the ends of the resonator. To minimize vibration losses to the anchor and maximize mechanical quality factor $\left(Q_{m}\right)$, these silicon beams must have a small cross section and must be matched to the acoustic quarter- 


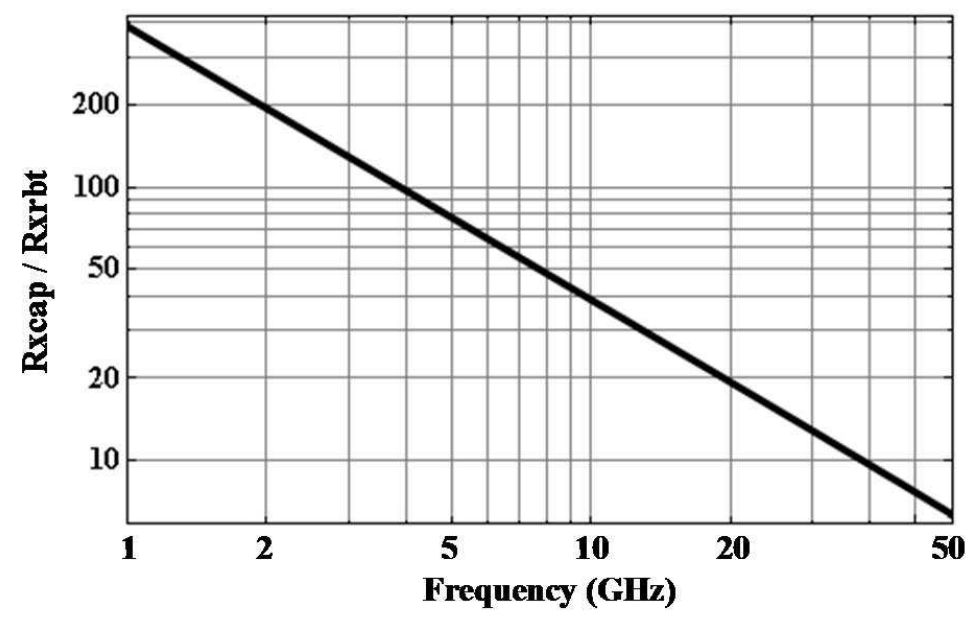

Figure 6.4: Relative performance of a capacitive resonator to the RBT scaling with frequency.

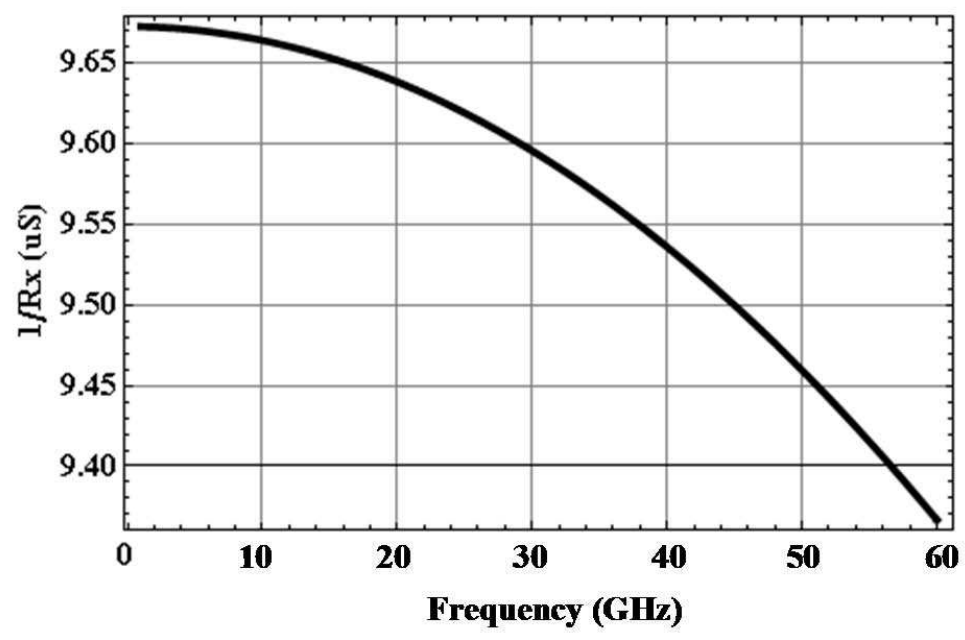

Figure 6.5: Frequency scaling of the motional admittance of the RBT. 
wavelength of the resonant frequency. This results in a parasitic series resistance $R_{\text {route }}$ at the input drive, where

$$
R_{\text {route }}=\rho \frac{L_{\text {route }}}{A_{\text {route }}}
$$

Here, $\rho_{S i}$ is the resistivity of the silicon, and $L_{\text {route }}$ and $A_{\text {route }}$ are the length and cross-sectional area of the routing beam, respectively. The series resistance in Equation 6.6 results in Johnson noise in the beam given by rms voltage fluctuations measured in a bandwidth $\Delta f$,

$$
v_{n, \text { route }}=\sqrt{4 k_{B} T R_{\text {route }} \Delta f}
$$

where $k_{B}$ is the Boltzmann constant and $T$ is the temperature of the beam. We assume a system temperature of $300 \mathrm{~K}$ and silicon resistivity of $0.1 \Omega . \mathrm{cm}$. Using Equation 6.7, the Johnson noise at the drive end of the resonator is given in Table 6.3 for a $1.5 \mathrm{GHz}$ and $10 \mathrm{GHz}$ bar. Coupled with the series resistance of

Table 6.3: Johnson Noise For Resonator Drive

\begin{tabular}{|c|c|c|c|c|c|c|}
\hline \hline$f_{\text {res }}$ & $L_{\text {res }}$ & $L_{\text {route }}$ & $w_{\text {route }}$ & $h_{\text {route }}$ & $R_{\text {route }}$ & $v_{\text {n,route }}$ \\
\hline $1.5 \mathrm{GHz}$ & $8.5 \mu \mathrm{m}$ & $4.4 \mu \mathrm{m}$ & $1 \mu \mathrm{m}$ & $2.5 \mu \mathrm{m}$ & $1.76 \mathrm{k} \Omega$ & $5.4 \times 10^{-9} \Delta f^{1 / 2}$ \\
\hline $10 \mathrm{GHz}$ & $1.3 \mu \mathrm{m}$ & $213 \mathrm{~nm}$ & $100 \mathrm{~nm}$ & $220 \mathrm{~nm}$ & $9.68 \mathrm{k} \Omega$ & $1.3 \times 10^{-8} \Delta f^{1 / 2}$ \\
\hline
\end{tabular}

the routing beam, the driving capacitive transducer

$$
C_{0}=\varepsilon_{f} \frac{w_{r e s} h_{r e s}}{g}
$$


behaves as a low-pass RC filter, attenuating noise above the cutoff frequency

$$
f_{\text {cutoff }}=\frac{1}{2 \pi R_{\text {route }} C_{0}}
$$

For a $15 \mathrm{~nm}$ thick silicon nitride transducer, Table 6.4 gives characteristic cutoff frequencies for $1.5 \mathrm{GHz}$ and $10 \mathrm{GHz}$ resonators.

Table 6.4: Cutoff Frequency At Drive Point

\begin{tabular}{|c|c|c|c|c|c|}
\hline \hline$f_{\text {res }}$ & $L_{\text {res }}$ & $w_{\text {res }}$ & $h_{\text {res }}$ & $C_{0}$ & $f_{\text {cutoff }}$ \\
\hline $1.5 \mathrm{GHz}$ & $8.5 \mu \mathrm{m}$ & $40 \mu \mathrm{m}$ & $2.5 \mu \mathrm{m}$ & $41 \mathrm{pF}$ & $219 \mathrm{MHz}$ \\
\hline $10 \mathrm{GHz}$ & $1.3 \mu \mathrm{m}$ & $500 \mathrm{~nm}$ & $220 \mathrm{~nm}$ & $0.45 \mathrm{fF}$ & $36 \mathrm{GHz}$ \\
\hline
\end{tabular}

The low cutoff frequency of the $1.5 \mathrm{GHz}$ resonator indicates some attenuation of the Johnson noise at the driving end of device. However, the driving voltage signal is attenuated by the same RC low-pass filter, so the signal to noise ratio is independent of the driving capacitor. The cutoff frequency for the 10 $\mathrm{GHz}$ resonator falls above the targeted resonant frequency, and the $\mathrm{RC}$ element attenuates neither Johnson noise nor signal in this case.

The Johnson noise calculated in Table 6.3 is a function of the noise bandwidth $\Delta f$. A voltage noise in a frequency interval between $f$ and $f+d f$ contributes to the motion of the resonator only if it that interval falls in the bandwidth of resonance. The relevant noise bandwidth for the driving noise is therefore $\Delta f=f / Q_{m}$. A reasonable estimate for $Q_{m}$ in silicon is $2 \times 10^{13} / f$, resulting in $\Delta f$ $=112 \mathrm{kHz}$ at $1.5 \mathrm{GHz}$ and $\Delta f=5 \mathrm{MHz}$ at $10 \mathrm{GHz}$. The Johnson noise contributing to mechanical vibrations is then

$$
\left.v_{n, \text { routel }}\right|_{1.5 \mathrm{GHz}}=1.8 \mu \mathrm{V}
$$




$$
\left.v_{n, \text { route }}\right|_{10 G H z}=28 \mu \mathrm{V}
$$

Typical values for the driving ac voltage $v_{\text {in }}$ are $0.1 \mathrm{~V}$, indicating a signal to noise ratio at the resonator drive of $\sim 10^{4} \sim 10^{5}$.

\subsubsection{Mechanical Thermal Noise}

A second source of thermal noise in the IDT silicon bar resonator is internal mechanical damping of the resonant mode. This internal friction gives rise to a noise force

$$
f_{n, \text { damp }}=\sqrt{4 k_{B} T b \Delta f}
$$

Here, $\mathrm{b}$ is the damping and can be expressed as

$$
b=\frac{\omega_{0} m_{e f f}}{Q_{m}}
$$

where $\omega_{0}$ is the resonant angular frequency and $m_{\text {eff }}$ is the effective mass of the resonant mode. Following the methods outlined in [35], the noise displacement inside the resonator due to mechanical damping is

$$
\begin{aligned}
z_{n, \text { damp }} & =\frac{f_{n, \text { damp }}}{k_{\text {eff }}} H(f) \\
& =\frac{\sqrt{4 k_{B} T b \Delta f}}{k_{e f f}} \frac{1}{1-\left(\frac{f}{f_{0}}\right)^{2}-i \frac{f}{Q_{m} f_{0}}} \\
& =\sqrt{\frac{4 k_{B} T \Delta f}{2 \pi f_{0} Q_{m} k_{e f f}}} \frac{1}{1-\left(\frac{f}{f_{0}}\right)^{2}-i \frac{f}{Q_{m} f_{0}}}
\end{aligned}
$$


The mode shape of longitudinal resonance for the nth harmonic of vibrations is given by

$$
u(x, t)=u_{0} \sin \left(\frac{n \pi}{L} x\right) e^{i \omega t}
$$

The effective mass of the resonator lumped at its point of maximum displacement is

$$
\begin{aligned}
m_{\text {eff }} & =\rho w h \frac{\int_{-L / 2}^{L / 2}\left(u_{0} \sin \left(\frac{n \pi}{L} x\right)\right)^{2} d x}{u_{0}^{2}} \\
& =\frac{1}{2} \rho w h L
\end{aligned}
$$

Using $k_{e f f}=\omega_{0}^{2} m_{e f f}$ in Equation 6.14 determines $z_{n, \text { damp }}$ for the resonant mode. Applying a driving signal $v_{\text {in }}$ across the drive dielectric excites the nth harmonic mode with maximum longitudinal displacement given by Equation 3.4. Using Equations 6.14 and 3.4, a 3rd harmonic longitudinal mode has signal to noise ratio of

$$
\begin{aligned}
& \left.\frac{u_{0}}{z_{n, \text { damp }}}\right|_{1.5 \mathrm{GHz}}=18,350 \\
& \left.\frac{u_{0}}{z_{n, \text { damp }}}\right|_{10 \mathrm{GHz}}=2,750
\end{aligned}
$$

At $60 \mathrm{GHz}$, the signal to noise ratio is 450 . The thermal noise due to mechanical damping does not pose a significant hindrance to the signal to noise ratio in the frequency range of interest. 


\subsubsection{Sensing Noise}

There are multiple methods for detecting vibrations in an IDT bar resonator. Below, we investigate noise contribution in the IDT resonator due to capacitive, piezoresistive, and MOSFET sensing.

\section{Capacitive Sensing}

The capacitive sensing setup for the IDT resonator is shown in Figure 6.6. The output dielectric transducer is subject to a bias voltage $V_{D C}$, as in the case of capacitive drive. Mechanical motion in the bar causes the dielectric film to expand and contract, resulting in a change of the sensing capacitor at the resonant frequency. This generates a measurable output current

$$
i_{\text {out }}=V_{D C} \frac{d C}{d u} \frac{d u}{d t}
$$

As in the case of capacitive drive, Johnson noise in the resistive routing beams dominates the output noise. Due to the symmetry of the device, $R_{\text {route }}$ is the same as calculated in Table 6.3 for the input noise. The resulting noise current is given by

$$
i_{n, \text { route }}=\sqrt{\frac{4 k_{B} T \Delta f}{R_{\text {route }}}}
$$

The noise bandwidth at the output is defined by the detector bandwidth. In the case of capacitive sensing as shown in Figure 6.6, the detector bandwidth is set by the network analyzer, and can be as low as a few $\mathrm{Hz}$ (at the expense of 


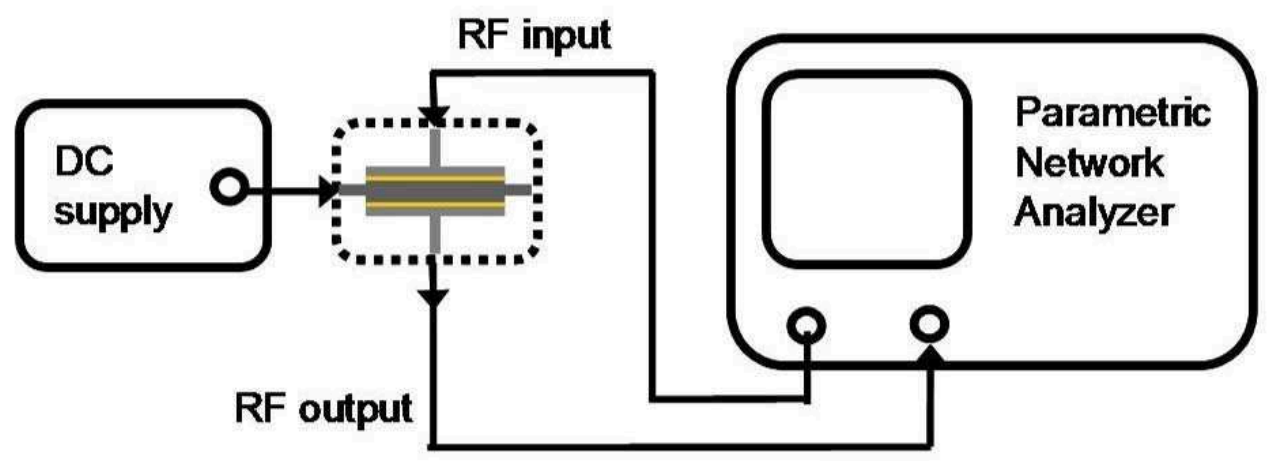

Figure 6.6: Schematic of measurement setup for a capacitively-sensed IDT resonator. The resonator body is biased to $V_{D C}$, while an RF excitation across one dielectric transducer drives resonance. An RF current is detected across the second dielectric transducer.

detection time). Assuming $\Delta f=30 \mathrm{~Hz}$, the noise current at the output is

$$
\begin{gathered}
\left.i_{n, \text { route }}\right|_{1.5 \mathrm{GHz}}=16.8 \mathrm{pA} \\
\left.i_{n, \text { route }}\right|_{10 \mathrm{GHz}}=7.2 \mathrm{pA}
\end{gathered}
$$

This results in a signal to noise ratio of $9.4 \times 10^{4}$ and $2.2 \times 10^{5}$ for the $1.5 \mathrm{GHz}$ and $10 \mathrm{GHz}$ resonances, respectively. 


\section{Piezoresistive Sensing}

A second sensing mechanism for IDT drive is piezoresistive detection. Elastic waves generated in the resonator modulate the mobility of carriers in the resonator body. Passing a drain current Id through the resonator, as shown in Figure 5.8, results in a modulated component of the drain current at the resonant frequency due to piezoresistive effect. (The change in resistance due to modulation of the carrier mobility is given by Equation 5.2). The piezoresistive transconductance is determined by

$$
g_{m}=\frac{d R}{R} \frac{I_{d}}{v_{g}}
$$

and can be extracted from the Y-parameters in the measurement setup of Figure 6.6 using Equation 5.4.

To avoid cancellation of the signal due to strain symmetry of the resonant mode, it is necessary to preferentially dope the regions close to the dielectric transducers, blocking current from passing through the central axis of the resonator body. As expected, there is a Johnson noise current associated with the series resistance intrinsic to the resonator body and routing,

$$
i_{n, \text { piezo }}=\sqrt{\frac{4 k_{B} T \Delta f}{R_{\text {body }}+2 R_{\text {route }}}}
$$

The $1.5 \mathrm{GHz}$ IDT resonator has a total resistance $\left(R_{\text {body }}+2 R_{\text {route }}\right)$ of $15.9 k \Omega$. In the case of the $10 \mathrm{GHz}$ resonator, the total resistance is $25 k \Omega$. For a detector bandwidth of $\Delta f=30 \mathrm{~Hz}$, this yields a piezoresistive Johnson noise of

$$
\left.i_{n, p i e z o}\right|_{1.5 \mathrm{GHz}}=5.6 \mathrm{pA}
$$




$$
\left.i_{n, \text { piezo }}\right|_{10 \mathrm{GHz}}=4.5 \mathrm{pA}
$$

For both frequencies, the signal to noise ratio for piezoresistive Johnson noise is $\sim 10^{6}$.

As in most semiconductor devices, flicker noise is also present in the case of piezoresistive sensing of the IDT resonator, and is related to the DC drain current flowing through the device. However, this noise follows a $1 / f$ frequency

trend, and is consequently a low-frequency effect. White noise sources like the thermal noise of Equation 6.22 dominate in the high-frequency regime of interest.

\section{MOSFET Sensing}

Finally, we consider the case of IDT drive in the resonator with MOSFET sensing. A schematic of this sensing configuration is illustrated in Figure 6.2. This resonator consists of an undoped region (light grey) and $n+$ doped regions (dark grey). One gate biases the active area into accumulation, such that no current flows, but a sheet charge is established to electrostatically drive mechanical resonance with an AC input voltage. The second gate biases the active region into strong inversion, defining a drain current Id which is modulated piezoresistively by elastic waves at resonance.

The amplitude of longitudinal resonance in this configuration is given in Equation 6.2, resulting in an output current defined in Equation 6.4. With $L_{\text {gate }}$ $=200 \mathrm{~nm}, \mu_{n}=300 \mathrm{~cm}^{2} / V s$ (effective mobility degraded by surface states), $V_{G}$ $=3 \mathrm{~V}, V_{D}=\left(V_{G} V_{T}\right)+0.1$, and $\mathrm{W}=1 \mu m$, the drain current $I_{d}=393 \mu A$ and the electrical transconductance of the MOSFET is $g_{m}=.70 \mu \mathrm{S}$. 
In the case of MOSFET sensing, several noise sources must be considered, including flicker noise, thermal noise, and shot noise. Flicker noise, or 1/f noise, plays a significant role in MOSFET devices. Two competing theories have been developed to explain flicker noise in transistors: Hooges mobility fluctuation theory and McWhorter's number fluctuation theory. In practice, an empirical fit is generally necessary to determine which effect dominates. For simplicity, we consider an approximation using mobility fluctuation theory [36],

$$
i_{n, \text { mob }}=\sqrt{\frac{q \alpha_{H} I_{d}^{2}}{h L_{\text {gate }} C_{o x}\left(V_{G}-V_{T}\right) f}}
$$

where $\alpha_{H}=2 \times 10^{-3}$ is Hooge's constant and $h$ is the thickness of the device layer comprising the resonator. For the resonator parameters specified above, the flicker current noise is plotted as a function of frequency in Figure 6.7. For all frequencies of interest, the pA noise current due to flicker noise is 5 orders of magnitude below the resonant signal $\left(\left.i_{\text {out }}\right|_{\text {FET }}=0.3 \mu A\right)$. Though the approximation in Equation ?? may be off by as much as 2 orders of magnitude, the signal to noise ratio due to flicker noise would still exceed 1,000.

As with capacitive and piezoresistive sensing, MOSFET sensing is subject to thermal noise. In the strong inversion regime, the drain-referred thermal noise current is given by [37]

$$
i_{n, \text { therm }}=\sqrt{4 k_{B} T\left(\frac{2}{3} g_{m}\right) \Delta f}
$$

This corresponds to $15 \mathrm{pA}$ in the case of the IDT MOSFET device. Shot noise, attributed to the random fluctuations of carriers flowing across a junction, contributes significantly to MOSFET noise in the subthreshold regime, where current flow in the channel is more discrete. However, thermal noise in the channel dominates in saturation, where the IDT MOSFET resonator is biased. 
Flicker Noise in MOSFET sensing of IDT resonator

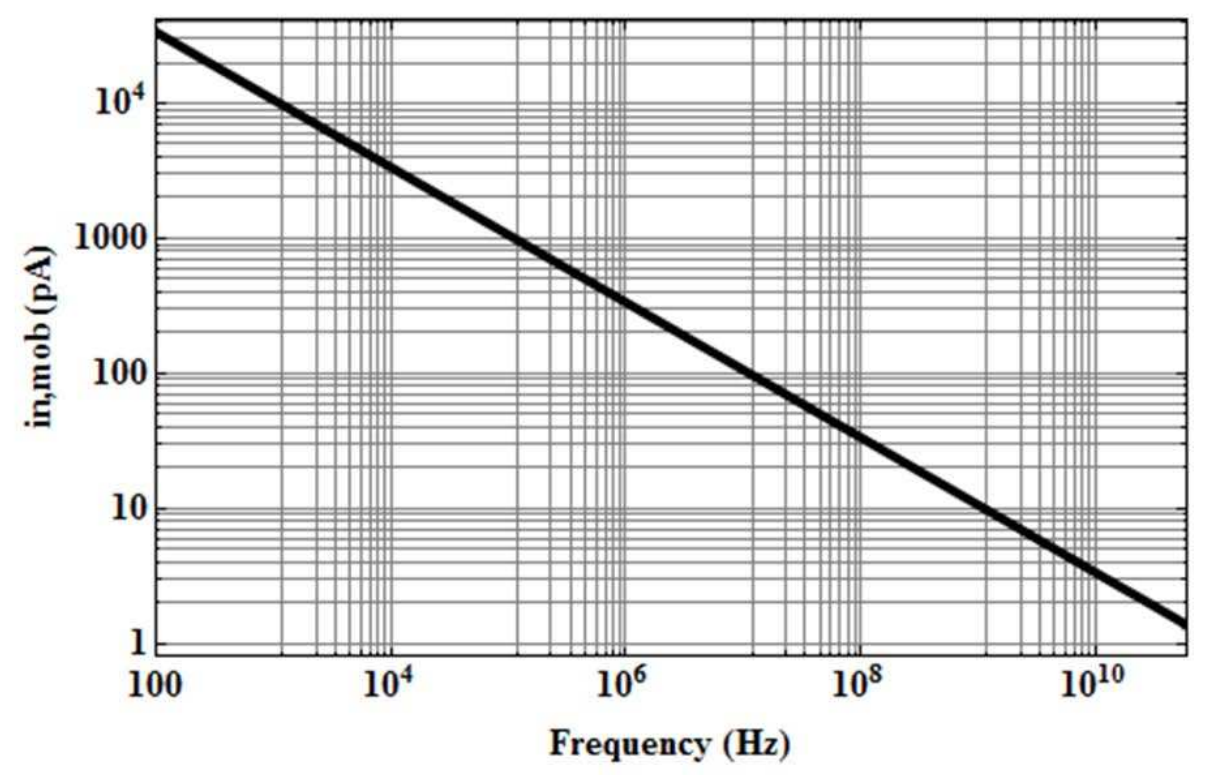

Figure 6.7: Flicker noise current in an IDT sensed using the MOSFET configuration. The $\mathrm{pA}$ amplitude of the noise current at frequencies of interest imply that flicker noise does not dominate the noise in the system.

\subsubsection{Conclusion}

Noise sources have been explored in IDT devices, including noise in the drive mechanism, internal to the resonant motion, and in the sense mechanism of the resonator. Multiple sensing transducers were investigated, including capacitive, piezoresistive, and MOSFET sensing. It is shown that all sensing transducers are viable for resonant detection, providing sufficient signal to noise ratios for practical applications. The primary source of noise in the IDT resonators is thermal. At intermediate frequencies, Johnson noise in the resistive routing beams to the resonator can dominate the noise of the system, and should be minimized in routing beam and resonator design. As we scale to $>60 \mathrm{GHz}$ fre- 
quencies, thermal noise due to mechanical damping in the resonator body becomes the dominant noise source, and should be considered carefully for highfrequency design. 


\section{CHAPTER 7}

\section{THE RESONANT BODY TRANSISTOR: FABRICATION}

Resonant Body Transistors and split-gate FinFETs were fabricated at the Cornell Nanoscale Science and Technology Facility (CNF). Figure 7.1 shows the full die configuration, including split-gate FinFETs, 10-20 GHz RBTs along (110) and (100) directions, S-O-T de-embedding structures as well as test structures.

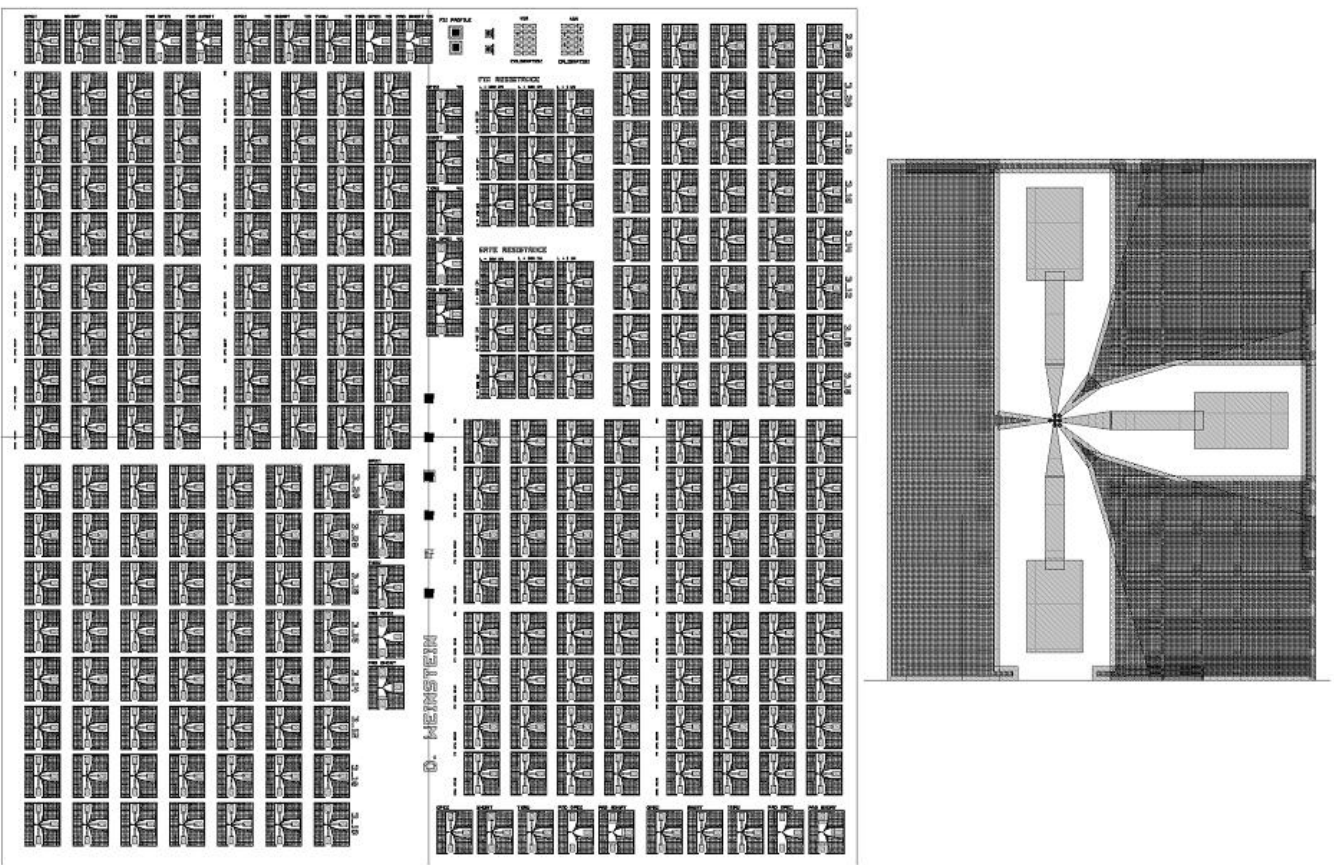

Figure 7.1: (left) Layout of die comprising RBTs ranging from 10 to $20 \mathrm{GHz}$ along (100) and (110) directions and split-gate FinFETs along (110) direction. (right) Zoomed in view of a single device showing metal pads and routing to the device.

The fabrication process implemented for RBT fabrication was inspired by the split-gate FinFET process developed by Dr. David Fried [38] in 2004, and is described in detail in this chapter. 


\subsection{ALN: Alignment Marks}

The process uses an SOI wafer with a $340 \mathrm{~nm}$ (100) lightly p-doped device layer and $400 \mathrm{~nm}$ buried oxide (BOX) layer, purchased from SOITEC. The device layer was oxidized to reduce its thickness to $230 \mathrm{~nm}$ and to provide a hard oxide mask for future processing steps. The wafer was thermally oxidized (Dry HCL Oxide) at $1100^{\circ} \mathrm{C}$ for 120 minutes.

The RBT fabrication process involves both Electron Beam (e-beam) and photolithography. Alignment marks for both lithography systems were written for all layers in one e-beam step. The e-beam used in this fabrication run was the JEOL JBX-9300FS system. Alignment marks were written in 495 PMMA 11\% in Anisole, spun at $1 \mathrm{krpm}$ for 60 seconds followed by a soft bake for 15 minutes at $170^{\circ} \mathrm{C}$. This corresponds to $2 \mu \mathrm{m}$ of PMMA. E-beam exposure of the alignment marks was done using a $5 \mathrm{~mA}$ beam of $100 \mathrm{keV}$ electrons, using a dose of 2200 $\mu \mathrm{C} / \mathrm{cm}^{2}$. The exposed wafer was developed in MIBK:IPA 1:3 for 75 seconds with agitation, rinsed with IPA and nitrogen dried.

Alignment marks were etched through the thermal oxide layer, device layer, BOX, and into the handle wafer to ensure their visibility through the many processing steps of the RBT run. The thermal oxide layer was first etched in ICP RIE (Oxford 100) using $100 \mathrm{sccm} \mathrm{CHF}_{3}$ and $2 \mathrm{sccm} \mathrm{O}_{2}$. The RF power was $15 \mathrm{~W}$ and the ICP power was $2500 \mathrm{~W}$. The chamber pressure was set to $5 \mathrm{mTorr}$, and the wafer chuck was maintained at $11^{\circ} \mathrm{C}$. The device layer was then etched in DRIE (Unaxis 770) using the OTRENCH recipe. The BOX layer was then etched

in the Oxford 100 using $\mathrm{CHF}_{3} / \mathrm{O}_{2}$ as for the thermal oxide layer. The PMMA was subsequently removed in Methyline Chloride with ultrasonication, rinsed with IPA, and Nitrogen dried. Finally, the alignment marks were etched $700 \mathrm{~nm}$ 
into the handle wafer in an ICP chlorine etch (Plasmatherm 770), using the top thermal oxide layer as a hard oxide mask. The wafer was cleaned in a Gasonics downstream asher (Aura $1000,4 \times$ recipe 2 ) to remove any polymer formed during the alignment mark etches.

\subsection{RX: Single-Crystal Silicon Fin And Inner Resonator}

The device layer of the wafer was defined in an RX e-beam mask, by which a negative resist is exposed and used to pattern the hard oxide mask layer. This top thermal oxide was then used to mask an ICP chlorine etch of the device layer. The negative e-beam resist used throughout the fabrication run was maN 2403, which has high resolution, good repeatability, and good adhesion on oxide.

The ma-N resist was spun on the wafer at $2.5 \mathrm{krpm} / 1 \mathrm{krpm} / \mathrm{sec}$ for $30 \mathrm{sec}-$ onds with no adhesion layer and soft-baked for 60 seconds at $90^{\circ} \mathrm{C}$. The RX layer was exposed in e-beam using both global and local alignment with a 1nA current and a dose of $1100 \mu \mathrm{C} / \mathrm{cm}^{2}$. The exposed resist was developed in MIF-300 for 75 seconds with gentle agitation, rinsed gently in multiple DI water baths, and blow dried with a very low nitrogen current.

The pattern was immediately transferred to the thermal oxide layer with a $\mathrm{CHF}_{3} / \mathrm{O}_{2}$ etch in the Oxford 100. Immediate pattern transfer after development of the e-beam resist is important to prevent nm-scale swelling of the ma- $\mathrm{N}$ resist and subsequent enlargement of critical features. Following the fluorine etch to define the RX hard oxide mask, the resist and polymers associated with the etch were removed in the Aura 1000. A top view of the RX component hard oxide mask of a $10 \mathrm{GHz}$ RBT can be seen in Figure 7.2(left). The RX pattern was then 
transferred to the device layer in an ICP chlorine etch using the Plasmatherm 770, followed by an additional oxygen clean in the Aura. The resulting hard oxide mask - device layer RX stack can be seen in Figure 7.2(right).

The side-walls of the device layer patterned in the RX mask ultimately form the active channel area of the split-gate FinFETs and of the FETs embedded in the Resonant Body Transistors. It is therefore imperative that this side-wall be as free of impurities and defects as possible. To this end, a thin layer of thermal oxide ( $\sim 3 \mathrm{~nm}$ using dry $\mathrm{HCL}$ oxide at $900^{\circ} \mathrm{C}$ for 3 minutes) was grown on the device layer after RX patterning, and stripped in dilute HF (100:1) for 45 seconds.
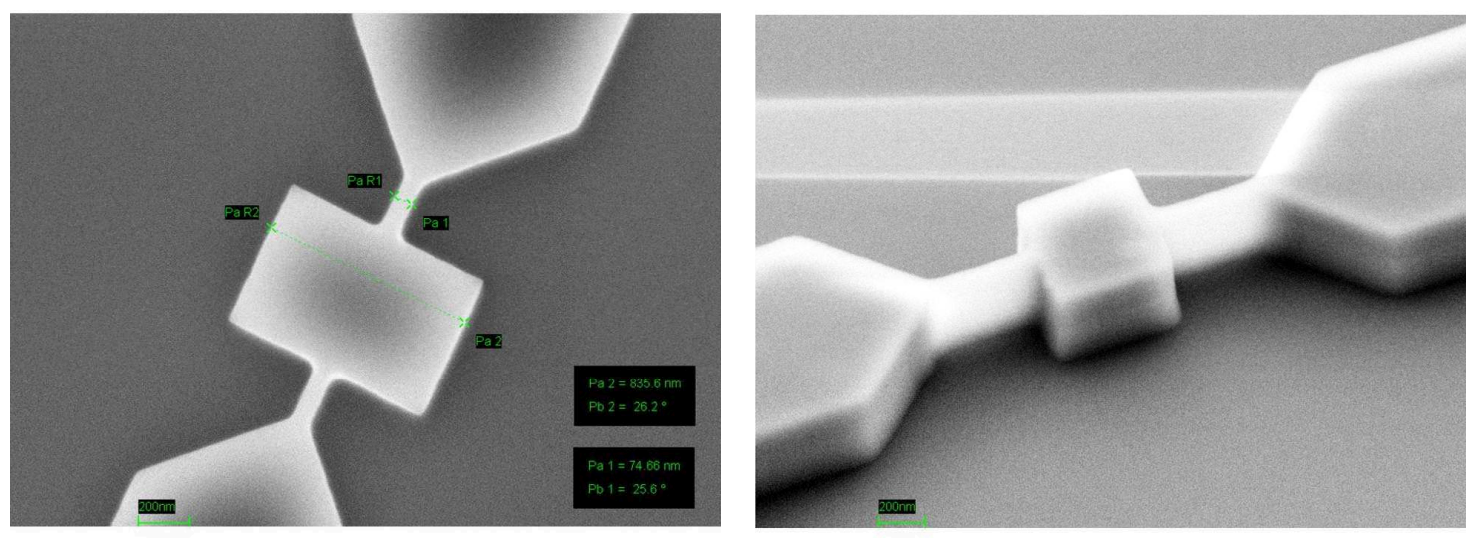

Figure 7.2: (left) Hard oxide mask (RX) to pattern the SOI device layer. (right) Device layer with remaining hard oxide mask etched in an ICP RIE chlorine etch.

\subsection{PC: Polysilicon Gate And Outer Resonator}

After the thin thermal oxide strip of the device layer side-walls, a $15 \mathrm{~nm}$ conformal stoichiometric nitride film was deposited to form the transduction (gate) dielectric. The film was deposited in an LPCVD furnace at $775^{\circ} \mathrm{C}$ for 4 minutes. 
Nitride deposition was followed immediately by a $430 \mathrm{~nm}$ film of $\mathrm{n}+$ doped LPCVD polysilicon, deposited at $590^{\circ} \mathrm{C}$ for 7 hours 10 minutes. The polysilicon was then annealed at $1050^{\circ} \mathrm{C}$ for 1 hour in an $\mathrm{N}_{2}$ ambient. A four-point-probe measurement of the polysilicon film yielded a sheet resistance $R_{s}=17.2 \Omega / \square$ corresponding to a polysilicon resistivity of $\rho_{\text {poly }}=7.3 \times 10^{-4} \Omega . \mathrm{cm}$.

The polysilicon was then planarized with Chemical Mechanical Polishing (CMP). The CMP tool used was a Strasbaugh 6EC with a Rodel IC1400 polyurethane pad. A Cabot SemiSperse P1000 polysilicon damascene slurry was mixed continuously with an electrical rotary tool to ensure homogeneity in the polishing slurry pumped to the pad. The polishing downward force was set to $7.5 \mathrm{psi}$ with a table rotation of $90 \mathrm{rpm}$, a chuck rotation of $30 \mathrm{rpm}$, and slurry pump speed of $150 \mathrm{~mL} / \mathrm{min}$.

Prior to polishing, the wafer was dipped in dilute HF (100:1) to remove the native oxide formed on the polysilicon since the etch rate of oxide is much slower than that of polysilicon using the P1000 slurry noted above. The wafer was then polished for 15 seconds. Without letting it dry, the wafer was transferred directly to a water bucket and taken to the chemical hoods for post-CMP clean. The wafer was transferred from the water bucket to a Standard Clean 1 bath (1 part $\mathrm{NH}_{4} \mathrm{OH}, 1$ part $\mathrm{H}_{2} \mathrm{O}_{2}, 6$ parts $\mathrm{DI} \mathrm{H}_{2} \mathrm{O}$ ) and ultrasonicated for 10 minutes. The water in the transfer bucket was changed, and the wafer was returned to the bucket without drying. The wafer was then transferred (again, without drying) from the water bucket to a Hamatech automatic post-CMP clean tool, scrubbed with a CMP clean rotary sponge, and spun dry. The wafer was then MOS cleaned at $70^{\circ} \mathrm{C}$ and dipped in dilute HF (100:1) for 20 seconds. The resulting planarized device is shown in Figure 7.3(left). The dark polysilicon region seen in the field area is removed from the devices, exposing the hard oxide mask 
of the RX pattern.
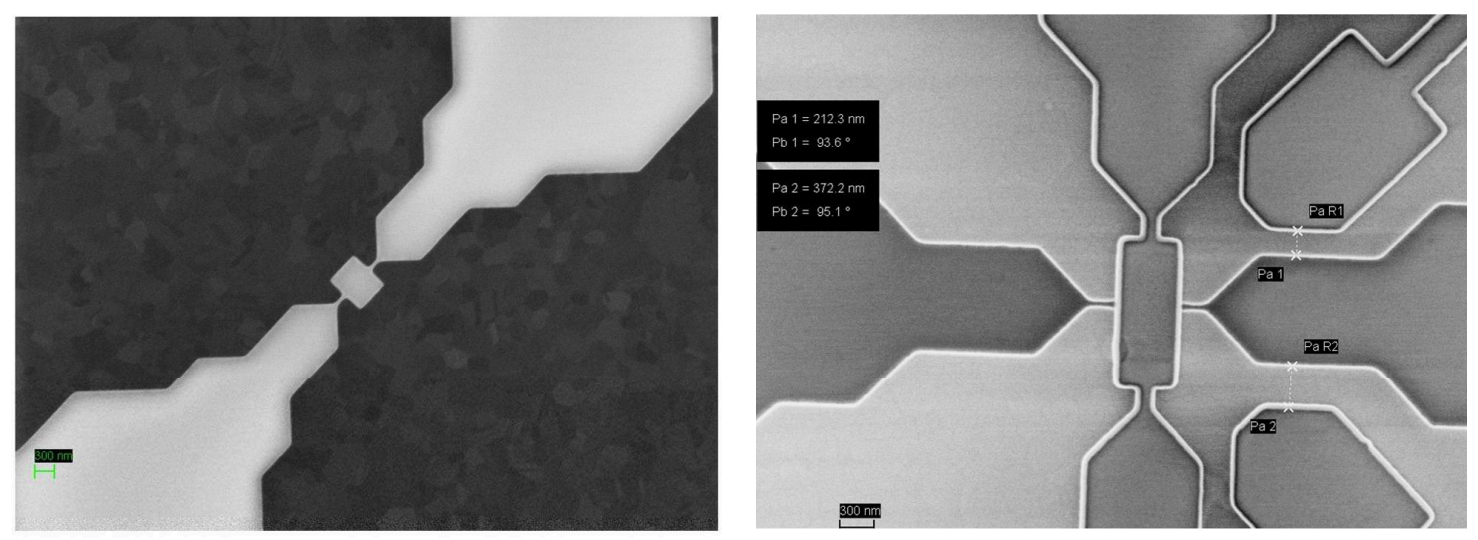

Figure 7.3: (left) Polysilicon planarization with an etch stop on the hard oxide mask used for device layer patterning. (right) Hard oxide mask (PG) patterning for poly gate definition and ion implant mask.

A $270 \mathrm{~nm}$ hard oxide mask layer for the PC mask was then deposited using PECVD undoped oxide (GSI N1.46 recipe) at $400^{\circ} \mathrm{C}$. The deposition involved two 40 second depositions, rotating the wafer $90^{\circ}$ half-way through to prevent any continuous pinholes from forming. The wafer was then annealed at $800^{\circ} \mathrm{C}$ in a nitrogen ambient to improve the quality of the film and reduce the risk of cracking in future high-temperature process steps. The wafer was loaded into the anneal furnace at $400^{\circ} \mathrm{C}$ (the deposition temperature of the PECVD oxide) and was ramped up to $800^{\circ} \mathrm{C}$ over the course of 2 hours. The wafer then sat at this anneal temperature for an additional 45 minutes, and unloaded from the furnace at $500^{\circ} \mathrm{C}$.

As with the RX ebeam exposure, ma-N ebeam resist was spun on the wafer at $2.5 \mathrm{krpm} / 1 \mathrm{krpm} / \mathrm{sec}$ for 30 seconds and soft baked at $90^{\circ} \mathrm{C}$ for 60 seconds. Using both global and local alignment, the PC mask was written in e-beam at 1nA with a dose of $1000 \mu \mathrm{C} / \mathrm{cm}^{2}$. The exposed resist was then developed in MIF300 for 75 seconds with gentle agitation, rinsed gently with DI water, and gently 
blow dried with a nitrogen gun.

The PC pattern was then transferred to the PECVD hard oxide mask layer in the Oxford 100. The tool conditions were irreversibly altered between the RX and PC hard oxide mask etches, and a new oxide etch recipe was necessary to reduce polymerization and side-wall angle characteristic of the altered ICP tool. The fluorine etch for the PC hard oxide mask used $90 \mathrm{sccm} \mathrm{CHF}_{3}, 5 \mathrm{sccm} \mathrm{O}$, and $10 \mathrm{sccm} \mathrm{CF}_{4}$. The chamber pressure was set to $5 \mathrm{mTorr}$, and an RF power of $15 \mathrm{~W}$ and ICP power of $2500 \mathrm{~W}$ were used. The wafer was then cleaned in the Aura followed by a Hot Piranha Hamatech clean. Figure 7.3(right) shows the PC hard oxide mask patterned on top of the planarized polysilicon and RX hard oxide mask.
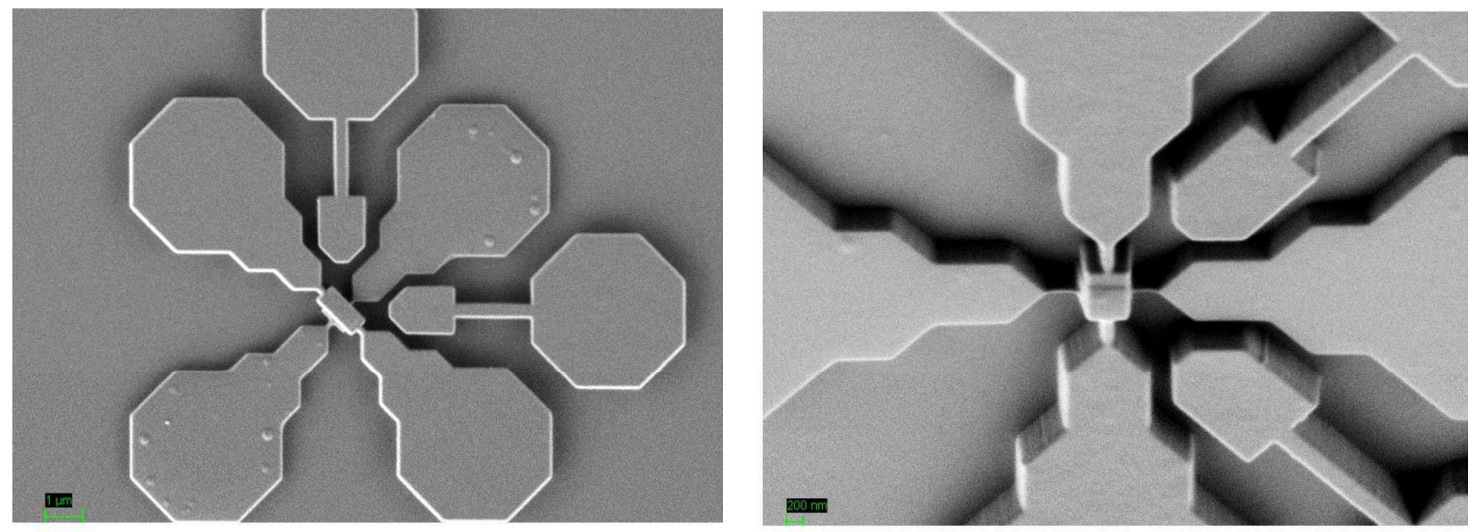

Figure 7.4: (left) Polysilicon etch in an ICP RIE chlorine etch to define gates and resonator body. (right) Removal of RX hard oxide mask for ion implant of device layer.

After an Aura and Hot Piranha clean of the wafer, the polysilicon was etched in an ICP chlorine etch in the Plasmatherm 770. The standard single-crystal silicon recipe for shallow chlorine etch had to be modified to reduce the selectivity of silicon to oxide, thereby preventing micro-masking at the polysilicon grain boundaries and the resulting black silicon. During the polysilicon etch, the 
PECVD oxide functions as a hard oxide mask for the gate and outer resonator definition. Meanwhile, the thermal oxide first grown on the device layer and used as a hard oxide mask for RX silicon etch (and as an etch stop during CMP) protects the patterned device layer and removes the polysilicon surrounding it. The wafer was then cleaned in the Aura and in Hot Piranha. Figure 7.4(left) shows the overlapping RX and PC regions of a $10 \mathrm{GHz}$ RBT. The intersection of the RX and PC masks indicates the position of the nitride dielectric films along the perimeter of RX.

\subsection{Ion Implant Of Device Layer}

While the polysilicon defining the FinFET gates and RBT outer resonator is highly doped, the source and drain regions defined in RX are lightly doped p-type silicon, and must be implanted to form conductive n-type contacts and routing to the device. The PC hard oxide mask functions as a implant mask for the active region of the FETs during this step.

To prepare the wafer for ion implant, the RX hard oxide mask over the source and drain regions was removed in $\mathrm{CHF}_{3} / \mathrm{O}_{2}$ fluorine etch using the Oxford 80 \# 2. This blanket etch resulted in thinning of the PC hard oxide mask, but left more than $100 \mathrm{~nm}$ of PC oxide on top of $100 \mathrm{~nm}$ of RX oxide over the active area of the device. Figure 7.4(right) shows the resulting device with RX oxide removed from the source and drain regions. The wafer was again cleaned in the Aura and Hot Piranha before implant.

The wafer was implanted by Core Systems, using a $1 \times 10^{15} 1 / \mathrm{cm}^{2}$ dose of Arsenic at $70 \mathrm{keV}$. The wafer was tilted at $10^{\circ}$ and rotated continuously about the tilt axis throughout the implant. The ion implant energy was chosen to pen- 
etrate deeply into the source and drain, but to stop in the PC and RX imlant mask over the active FET area. The tilted rotation during implant assisted in uniform doping through the thickness of the device layer (particularly in the source and drain suspension beams to the device) and allowed for penetration of the As ions under the implant mask to reduce series resistance from source to drain. The dopant was activated in a furnace anneal in $\mathrm{N}_{2}$ ambient, ramping from $700^{\circ} \mathrm{C}$ to $1000^{\circ} \mathrm{C}$ over the course of 1 hour and annealing at $1000^{\circ} \mathrm{C}$ for 30 minutes.

\subsection{Metallization}

Metallization for the RBT fabrication run was achieved using i-line photolithography in a GCA Autostep 200 5x stepper. All photolithography 5 inch masks for the RBT run were made in a Heidelberg DWL 66 laser writer. The i-line photoresist used in this process was SPR220-3.0. All resist coatings were spun after a P20 adhesion layer at $4 \mathrm{krpm} / 1 \mathrm{krpm} / \mathrm{sec}(2 \mu m$ thick), and soft baked at $115^{\circ} \mathrm{C}$ for 90 seconds. Exposure doses, post-exposure bake, and develop recipes varied from mask to mask and are discussed below.

\subsubsection{SUB And CA: FOX Cladding And Contact Vias}

Following the ion implant and anneal, a $1.1 \mu \mathrm{m}$ thick layer of GSI PECVD field oxide (FOX) was deposited to reduce parasitic capacitance between metal probe pads and the substrate wafer. Including the $270 \mathrm{~nm}$ of the BOX layer remaining after processing, the total oxide layer between the substrate and metallization 
layer is $1.37 \mu \mathrm{m}$. It should be noted that the PECVD oxide was not entirely conformal and left some non-uniform covered trenches along the perimeter of the device (Figure 7.5).
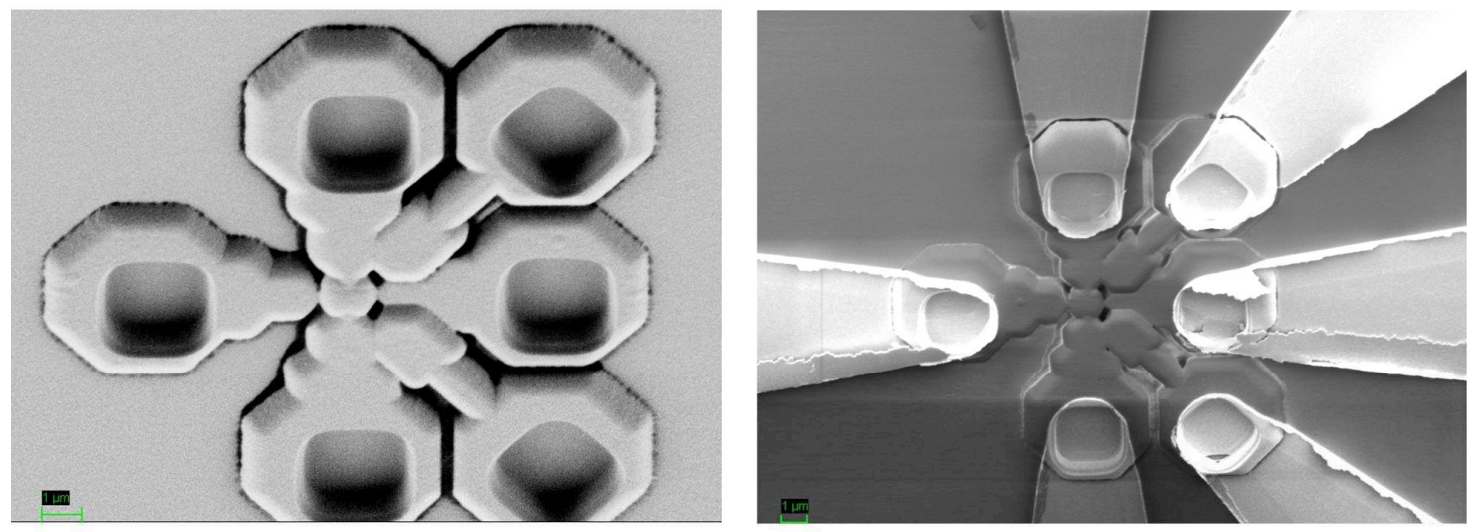

Figure 7.5: (left) Contact holes etched into field oxide for electrical connection to the device. (right) Nickel silicide and metallization using conformal e-beam evaporation and liftoff.

Contact holes to the device (CA) and to the substrate wafer (SUB) were then opened up through the FOX and BOX layers. After spinning the SPR220-3.0 resist as described above, the wafer was exposed in the Autostep 200 using both global and local alignment with an exposure time of 0.2 seconds corresponding to $40 \mathrm{~mJ} / \mathrm{cm}^{2}$ using $365 \mathrm{~nm}$ illumination. The wafer was post-exposure baked at $115^{\circ} \mathrm{C}$ for 90 seconds and developed with MIF-300 in a 120 second doublepuddle recipe using a Hamatech automated wafer developer. While the standard develop recipe for SPR220-3.0 calls for 60 seconds development in MIF300 , this over-development was necessary to ensure that the $2 \mu m \times 2 \mu m$ contact holes in CA were fully developed in the $2 \mu m$ thick resist.

The wafer was descummed in the Oxford 80 \# 2 in an oxygen plasma using $50 \mathrm{sccm}$ of $\mathrm{O}_{2}$ at 30 mTorr with a $100 \mathrm{~W}$ RF power for 2 minutes. This corresponds to an anisotropic $140 \mathrm{~nm}$ etch of the resist, ensuring cleared features 
even the small contact CA holes. The FOX was then etched in the Oxford 80 \# 2 to expose the substrate in SUB regions and the RX device layer and PC polysilicon layers in CA regions of the mask. Figure 7.5(left) shows the contact CA vias through the FOX, exposing RX and PC silicon underneath. The wafer was then cleaned using the Aura asher and a Hot Piranha clean.

\subsubsection{M1: Nickel Silicide}

The exposed silicon regions from the SUB and CA etch were then silicided to form ohmic contacts for metallization. $2 \mu m$ of SPR220-3.0 were first spun onto the wafer and soft-baked as previously described. An inverse-tone mask for the metal probe pads and routing to the device (M1) was then exposed on the wafer using global and local alignment in the Autostep 200 with an exposure time of 0.325 seconds. The wafer was then placed in a Yes 58-SM image reversal oven at $90^{\circ} \mathrm{C}$, followed by a flood exposure for 60 seconds in a deep-UV light source (ABM contact aligner). The resist was developed in MF-321 for 70 seconds, rinsed in DI water and blow dried in nitrogen. The wafer was descummed in the Oxford 80 \# 2 in a $50 \mathrm{scccm} \mathrm{O}_{2}$ plasma at $100 \mathrm{~W}$ and $30 \mathrm{mTorr}$ for 1 minute 30 seconds.

Immediately prior to evaporation of the Nickel used to form the desired silicide, the wafer was dipped in a Buffered Oxide Etch (BOE 30:1) for 30 seconds to remove any native oxide formed on the exposed silicon. A $40 \mathrm{~nm}$ thick Nickel layer was deposited at $0.8 \AA / \mathrm{sec}$ in CHA Mark 50 e-beam evaporator at the Cornell Nanobiotechnology Center (NBTC). This evaporator provides a rotating substrate holder for conformal deposition to cover the $>1 \mu m$ step heights of the FOX layer to the silcon device and substrate. 
After Ni evaporation, lift-off of the M1 mask resist was performed with the wafer suspended upside down in an acetone bath with agitation (but no ultrasonication). Lift-off of the $\mathrm{Ni}$-coated resist was made possible despite the conformal deposition due to the $2 \mu \mathrm{m}$ thickness of the resist and the angled sidewalls of the resist due to image reversal. The wafer was rinsed in IPA and blow dried in nitrogen.

The wafer was annealed in an AG 610 Rapid Thermal Anneal (RTA) at $500^{\circ} \mathrm{C}$ for 30 seconds using a thermocouple sensor with feedback to ensure temperature stability at low annealing temperatures. Three calibration runs were performed before the final anneal, and the wafer was removed after the chamber cooled to $75^{\circ} \mathrm{C}$. After silicide formation, the residual Ni not consumed to form the silicide was stripped in a Hot Piranha clean. A four-point probe measurement on a blanket $\mathrm{Ni}$ silicide wafer yielded a silicide sheet resistance of 0.99 $\Omega / \square$. An XPS measurement of the blanket silicide, shown in Figure 7.6 shows no Au contamination to the sample.

\subsubsection{M1: Nickel Metallization}

The same lift-off process as was used for the Ni evaporation for silicide was then repeated for the final metallization step. Unlike for the silicide step, no BOE strip was performed prior to evaporation since NiSi is attacked by Hydrofluoric Acid. For the final metallization layer, $120 \mathrm{~nm}$ of $\mathrm{Ni}$ were deposited in the $\mathrm{CHA}$ evaporator at $0.8 \AA / \mathrm{sec}$. This was followed by an acetone lift-off with agitation, with short 5 second bursts of ultrasonication to remove ribbons of $\mathrm{Ni}$ formed on the resist side-wall during conformal $\mathrm{Ni}$ deposition. Figure 7.5(right) shows the final Ni routing through the contact vias to the silicon device. The bright 


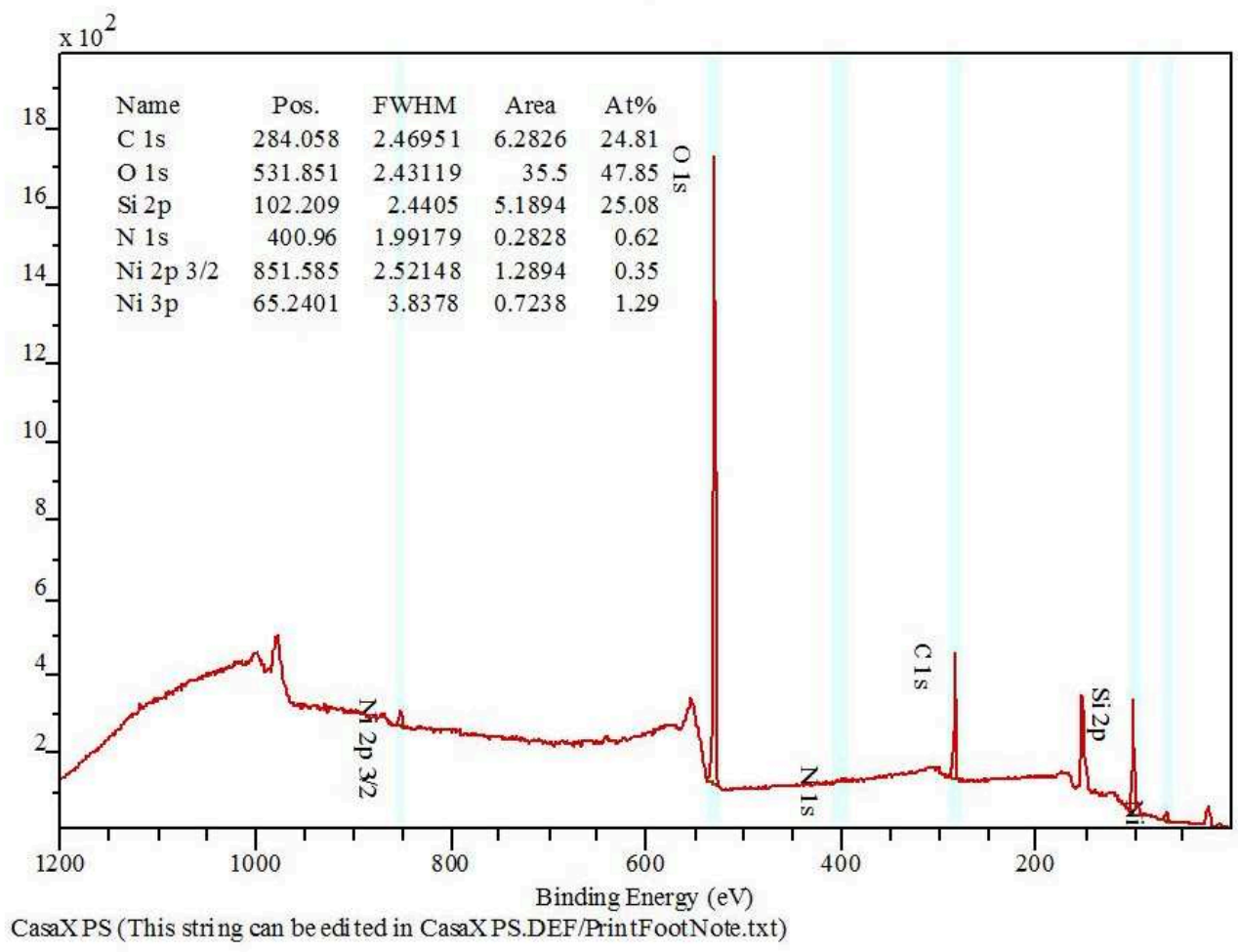

Figure 7.6: XPS scan of a Ni silicide sample indicating no Au contamination or other impurities during silicide formation.

appearance of the source, drain, and ground leads in the scanning electron micrograph indicate good electrical connectivity to the wafer substrate and across the device. Alternately, the darkened appearance of the two gate leads implies a good quality nitride dielectric layer isolating the gates from the source and drain.

\subsection{REL: Release}

With Ni metallization complete, the devices were ready to be released. A $2 \mu \mathrm{m}$ layer of SPR220-3.0 was first spun on the wafer and soft-baked. Using an ex- 
posure of $0.2 \mathrm{sec}$ in the Autostep 200, $2 \mu \mathrm{m} \times 2 \mu \mathrm{m}$ windows were exposed in the resist over the devices. As with the CA/SUB mask, the wafer was post-baked at $115^{\circ} \mathrm{C}$ for 90 seconds, followed by a Hamatech 120 second double-puddle develop in MIF300. The wafer was descummed in an Oxford $80 \# 2 \mathrm{O}_{2}$ plasma at $30 \mathrm{mT}$ and $100 \mathrm{~W}$ RF power for 2 minutes. $1 \mu \mathrm{m}$ of the FOX layer on top of the devices was then etched in $\mathrm{CHF}_{3} / \mathrm{O}_{2}$. The FOX layer was not removed completely to protect the nitride dielectric transducer from the fluorine etch. An additional 6 minutes of $\mathrm{O}_{2}$ plasma at $30 \mathrm{mT}$ and $100 \mathrm{~W}$ were then used to remove any polymer formed on the wafer during the fluorine etch. The remaining resist was stripped in acetone with an IPA rinse.

The REL mask in SPR220-3.0 was again spun, exposed, developed, and descummed, leaving the majority of the wafer coated in resist with $2 \mu m \times 2 \mu m$ windows exposing the devices for release. The wafer was cleaved by hand into individual dies.

The final release step was performed in a Buffered Oxide Etch (BOE 6:1) for 5.5 minutes with light agitation. The dies were transferred to fresh DI water baths 4 times, ensuring that the water did not roll off the dies as they were lifted out of each bath. The dies were then transferred to a bath with 50\% DI water / $50 \%$ acetone, then transferred to a pure acetone bath to remove the resist used to mask the $\mathrm{BOE}$ wet etch.

The dies were then transferred successively to a $75 \%$ acetone / $25 \%$ methanol bath, a 50\% acetone / 50\% methanol bath, a 25\% acetone / 75\% methanol bath, and finally a pure methanol bath. The dies remained in each bath for at least 5 minutes to allow for removal of all acetone from the device trenches. The dies were then immersed in methanol in a Tousimis Critical Point Dryer (CPD), with a cool setting of 0.5 , a fill setting of 1.0 , purge-vent set at 0.25 , and a bleed 

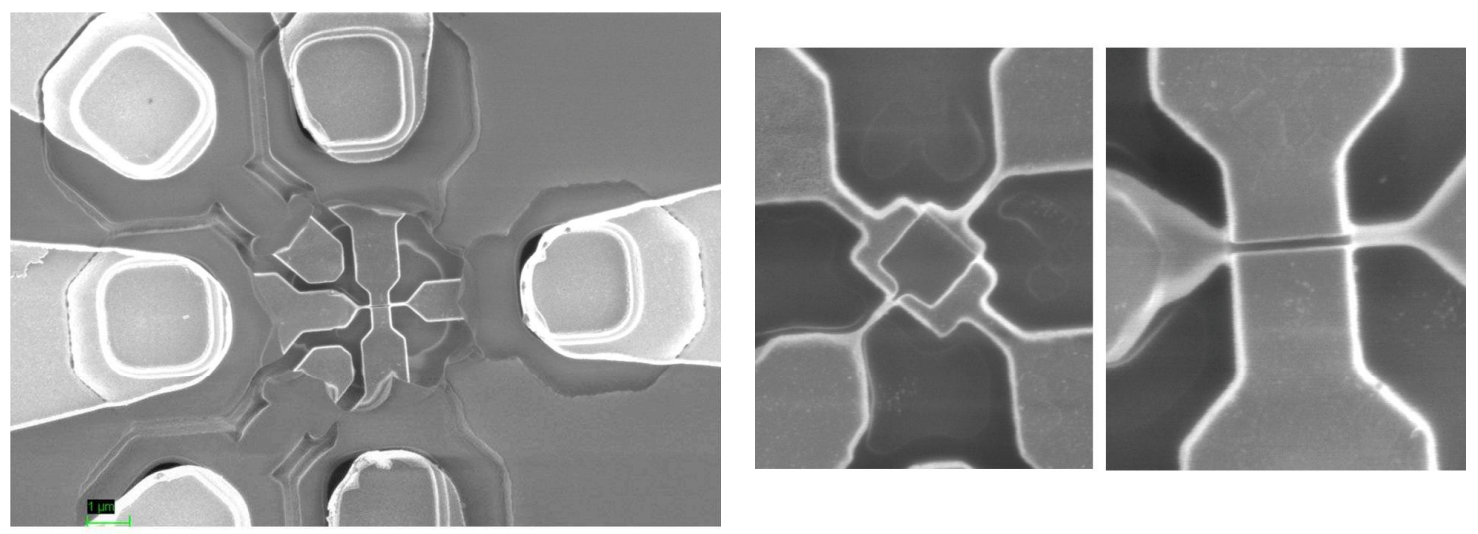

Figure 7.7: (left) Released FinFET showing metal contacts and release window. (right) Released RBT and FinFET. The darker region in silicon indicates the undoped active area of the device.

setting of 0.25 . The CPD chamber was purged for 30 minutes before reaching critical point. A scanning electron micrograph of the released devices is shown in Figure 7.7. 
CHAPTER 8

THE RESONANT BODY TRANSISTOR: MEASURED RESULTS

\subsection{Measurement Setup}

\subsubsection{De-embedding}

Detection of the RBT and Split-Gate FinFET requires high-frequency measurement ranging over tens of GHz. However, to probe each resonator and FinFET individually, large probe pads and routing to each device results in significant capacitance which dominates the measured signal. The parasitic capacitance and inductance of the probe pads and routing can be subtracted from the measured device using de-embedding structures on-chip. Models of the deembedding structures used for the RBT and FinFET measurements are shown in Figure 8.1.

The de-embedding algorithm implemented in these measurements was developed in [39], and is outlined below.

1. Measure S-parameters of device under test (DUT), open, short, and through structures $\left[S^{D U T}\right],\left[S^{O P E N}\right],\left[S^{S H O R T}\right],\left[S^{T H R U}\right]$.

2. Convert $\left[S^{O P E N}\right] \longrightarrow\left[Y^{O P E N}\right],\left[S^{S H O R T}\right] \longrightarrow\left[Y^{S H O R T}\right]$.

3. Subtract $\left[Y^{S-O}\right]=\left[Y^{S H O R T}-Y^{O P E N}\right]$.

4. Convert $\left[Y^{S-O}\right] \rightarrow\left[Z^{S-O}\right]$.

5. Calculate ABCD matrices of the input and output pads (PAD1 and PAD2, 

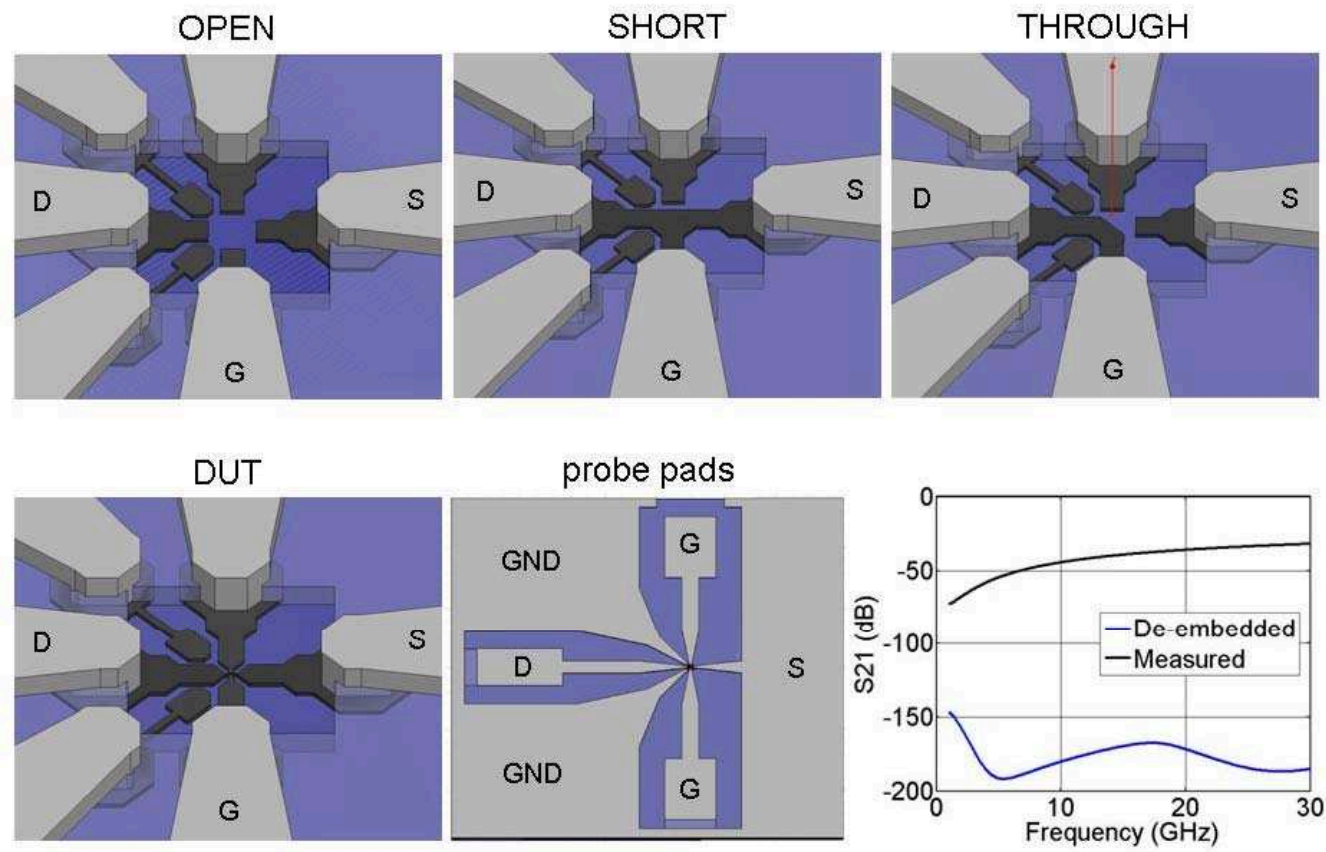

Figure 8.1: De-embedding structures fabricated on-chip to subtract parasitic capacitance and inductance of the probe pads and routing, lowering the measurement floor.

respectively) to the device:

$$
\begin{aligned}
& {\left[A^{P A D 1}\right]=\left[\begin{array}{cc}
1 & Z_{P A D} \\
Y_{P A D} & 1+Z_{P A D} Y_{P A D}
\end{array}\right]} \\
& {\left[A^{P A D 2}\right]=\left[\begin{array}{cc}
1+Z_{P A D} Y_{P A D} & Z_{P A D} \\
Y_{P A D} & 1
\end{array}\right]}
\end{aligned}
$$

where $Y_{P A D}=Y_{11}^{O P E N}+Y_{12}^{O P E N}$ and $Z_{P A D}=Z_{11}^{S-O}-Z_{12}^{S-O}$.

6. Convert $\left[S^{T H R U}\right] \longrightarrow\left[A^{T H R U}\right]$. 
7. Calculate

$$
\begin{array}{r}
{\left[A^{I N T}\right]=\left[A^{\text {PADI }}\right]^{-1}\left[A^{T H R U}\right]\left[A^{\text {PAD2 }}\right]^{-1}} \\
{\left[A^{I N T}\right] \longrightarrow\left[S^{I N T}\right]} \\
Z_{C} \equiv \pm Z_{0} \sqrt{\frac{\left(1+S_{11}^{I N T}\right)^{2}-\left(S_{21}^{I N T}\right)^{2}}{\left(1-S_{11}^{I N T}\right)^{2}-\left(S_{21}^{I N T}\right)^{2}}} \\
\kappa=-\frac{1}{\ell} \ln \left[\left(\frac{1-\left(S_{11}^{I N T}\right)^{2}+\left(S_{21}^{I N T}\right)^{2}}{\left.2 S_{21}^{I N T} \pm \kappa\right)^{-1}}\right]\right. \\
\kappa=\sqrt{\frac{\left(1-\left(S_{21}^{I N T}\right)^{2}+\left(S_{11}^{I N T}\right)^{2}\right)^{2}-\left(2 S_{11}^{I N T}\right)^{2}}{\left(2 S_{21}^{I N T}\right)^{2}}}
\end{array}
$$

8. Calculate $\mathrm{ABCD}$ matrices $\left[A^{I N T 1}\right],\left[A^{I N T 2}\right]$ using input and output interconnect lengths $\ell_{1}, \ell_{2}$

$$
\left[\begin{array}{ll}
A & B \\
C & D
\end{array}\right]=\left[\begin{array}{cc}
\cosh (\gamma \ell) & Z_{C} \sinh (\gamma \ell) \\
\frac{1}{Z_{C}} \sinh (\gamma \ell) & \cosh (\gamma \ell)
\end{array}\right]
$$

9. Calculate

$$
\begin{aligned}
{\left[A^{I N}\right] } & =\left[A^{P A D 1}\right]\left[A^{I N T 1}\right] \\
{\left[A^{\text {OUT }}\right] } & =\left[A^{I N T 2}\right]\left[A^{\text {PAD2 }}\right]
\end{aligned}
$$

10. Convert $\left[S^{D U T}\right] \longrightarrow\left[A^{D U T}\right]$.

11. Calculate $\left[A^{D}\right]=\left[A^{I N}\right]^{-1}\left[A^{\text {DUT }}\right]\left[A^{\text {out }}\right]^{-1}$.

12. Convert $\left[A^{I N}\right] \longrightarrow\left[S^{D}\right]$.

The resulting $\left[S^{D}\right]$ matrix corresponds to the 2-port frequency response of the device de-embedded from the probe pads and routing. It should be noted that 
de-embedding using the through structure does not remove the series resistance of the suspension beams to the RBT or the FinFET, but does eliminate the contact resistance between the metal and silicon layers.

\subsubsection{RF Measurement}

The devices were tested in a two-port configuration at room temperature in a vacuum probe station using a network analyzer. A schematic of the measurement setup is shown in Figure 8.2.

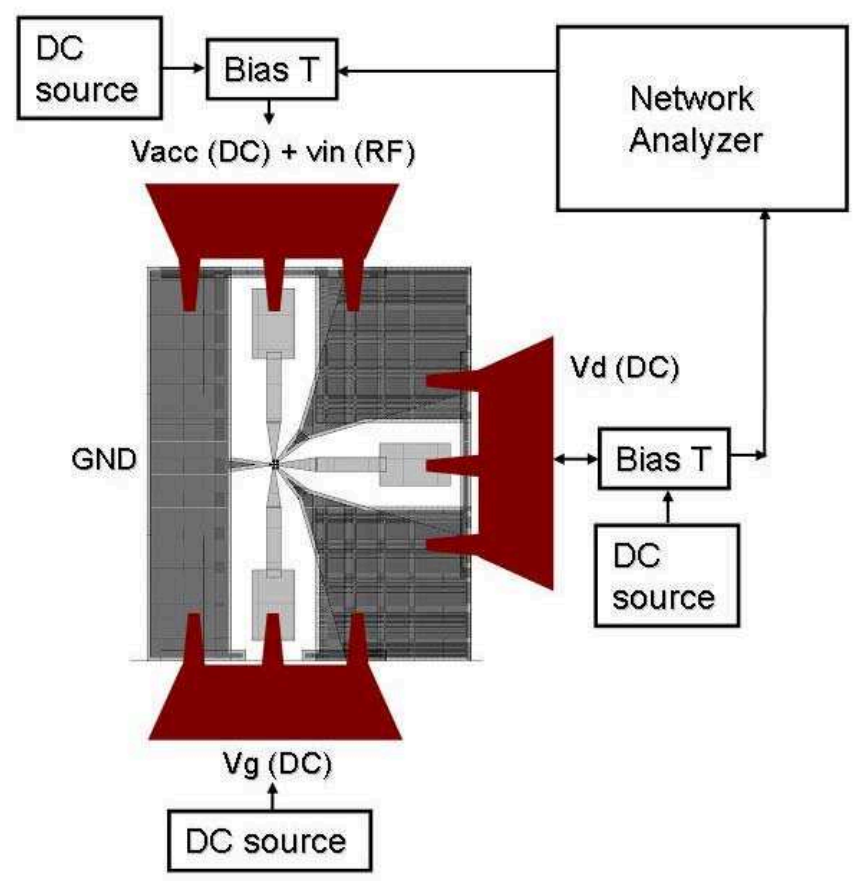

Figure 8.2: Measurement setup for the resonant body transistor.

As outlined in Figure 6.2, there are a couple of restrictions on the bias volt- 
ages to the RBT to achieve maximum transduction force while operating the sensing FET in saturation. A $5 \mathrm{~V}$ bias was applied at the inversion gate. This restricted the drain voltage $V_{D}>V_{G}-V_{T}$, set at $4.5 \mathrm{~V}$. To ensure that the drive gate was biased into accumulation, $V_{A C C}>V_{D}-V_{G}$ was set at $-0.5 \mathrm{~V}$. A $-20 \mathrm{dBm}$ input signal was superimposed on $V_{A C C}$ to induce a time-varying force across the transduction dielectric. This AC signal corresponds to $v_{i n} \sim 0.5 \mathrm{~V}$ at $12 \mathrm{GHz}$. It should be noted that while the accumulation bias voltage $V_{A C C}$ is only $0.5 \mathrm{~V}$, the total voltage across the drive dielectric is defined $V_{P}(x) \equiv V_{A C C}-V_{S}(x)$, where $V_{s}(x)$ is the surface potential at a position $x$ along the RBT. The voltage drop varies between $5 \mathrm{~V}$ in the drain region to $0.5 \mathrm{~V}$ in the source region. Therefore, in the majority of the transduction area, $V_{P} \gg v_{i n}$, and the linearisation made for Equation 1.2 from Equation 1.1 remains valid.

The devices were tested in vacuum to prevent ionization of air in the fringe fields near the dielectric, where $5 \mathrm{~V}$ were applied across a $15 \mathrm{~nm}$ gap. The vacuum also prevented adsorption of molecules onto the surface of the resonator over time, which can degrade the quality factor.

After de-embedding the device from the probe pads and routing, the transconductance is obtained from the Y-parameters, $g_{m}=Y_{21}-Y_{12}$, as in the case of conventional transistor measurements.

\subsection{Experimental Results}

To test the performance of the transistor sensing in the RBT, a Split-Gate FinFET was first measured tying both gates together and biasing the device at $V_{G}=5$ $\mathrm{V}$ into strong inversion. The resulting frequency response of the FinFET is presented in Figure 8.3. The FinFET tested has a fin width of $50 \mathrm{~nm}$ and a gate 
length of $L_{\text {gate }}=500 \mathrm{~nm}$. The cut-off frequency of the FinFET is $f_{T} \sim 42 \mathrm{GHz}$.

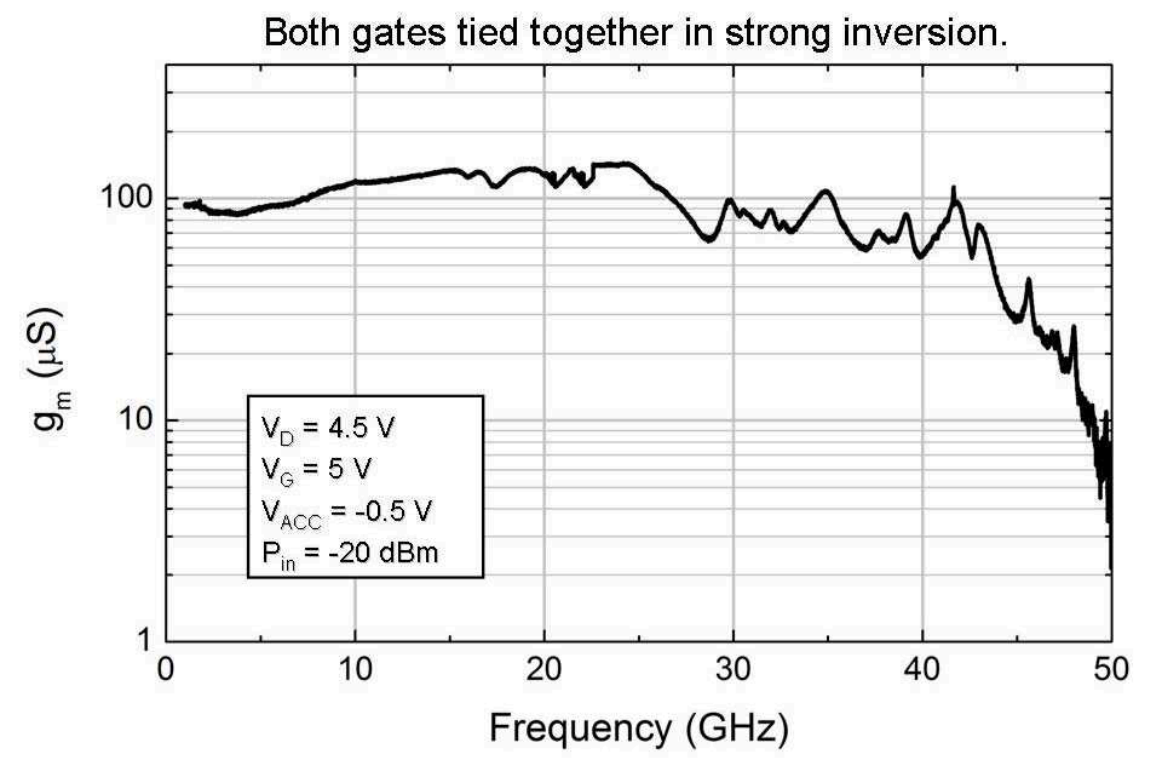

Figure 8.3: De-embedded transconductance measurement of a Split-Gate FinFET with both gates tied together and biased into strong inversion. The fin width is $50 \mathrm{~nm}$ and gate length is $500 \mathrm{~nm}$, with a $\mathrm{W} / \mathrm{L}$ ratio of $\sim 0.4$.

Because the FinFET is freely suspended, it may undergo mechanical resonance at high frequencies corresponding to longitudinal modes in the structure. The high-Q peaks at 46 and $48 \mathrm{GHz}$ have $f . Q$ products of $\sim 1 \times 10^{13}$, on par with the IDT longitudinal resonators demonstrated at lower frequency.

The Resonant Body Transistors were then tested in the configuration outlined in §8.1.2. The de-embedded frequency response of an $11.7 \mathrm{GHz} \mathrm{RBT}$ is shown in Figure 8.4. The electromechanical $Q$ of the RBT is 1831, resulting in an $f . Q$ product of $2.1 \times 10^{13}$.

There are several points to note regarding the performance of the RBT. As seen in Figure 7.7, the source and drain of the RBT are $\sim 30 \mathrm{~nm}$ wide, resulting in a non-negligible series resistance which adversely affects the transconduc- 


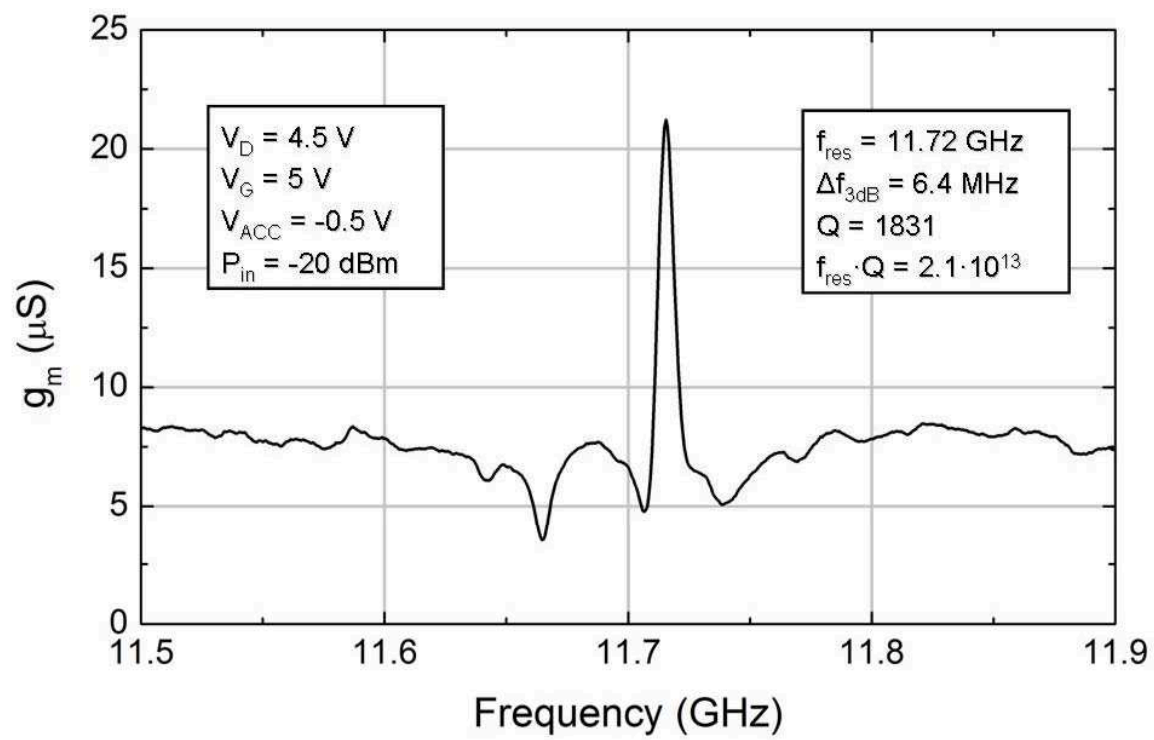

Figure 8.4: Measured RBT frequency response at its 3rd harmonic longitudinal resonance.

tance of the device. Second, there is a $\sim 100 \mathrm{~nm}$ misalignment in the RBT in the direction perpendicular to longitudinal vibrations. This misalignment results in a small region in which both accumulation and inversion gates have a strong effect on the channel. The electrical contribution of $v_{\text {in }}$ applied to the accumulation gate generates a back-gate effect on the RBT, contributing to an off-resonance transconductance floor which is higher than desired. Furthermore, the misalignment results in a component of the transducer forcing acoustic waves perpendicular to the designed longitudinal axis. This serves to de- $Q$ the longitudinal mode relative to its ideal operational state. The DC drain current through the RBT was $35 \mu \mathrm{A}$, dissipating less than $160 \mu \mathrm{W}$ in the resonator.

Nonetheless, the $11.7 \mathrm{GHz}$ resonance of the RBT is the highest acoustic frequency measured in a silicon resonator to date, breaking the record $6.2 \mathrm{GHz}$ resonance presented in $\S 5.1$. The resonant peak in Figure 8.4 demonstrates a 
clear signal of over $13 \mu \mathrm{S}$ above the background signal, which can be further improved with better device alignment. The $f . Q$ product of $2.1 \times 10^{13}$ is on par with the capacitive IDT resonators designed at lower frequency, and can also be improved with better alignment and with reduced series resistance to the RBT. This first demonstration of a Resonant Body Transistor, using internal dielectric drive and FET sensing in a bulk-mode resonator, promises the capability of scaling micro-fabricated resonators to multi-GHz frequencies. The RBT was fabricated side-by-side with Split-Gate FinFETs, indicating the capacity of these resonators for CMOS integration FEOL fabrication. 


\section{CHAPTER 9}

\section{FUTURE DIRECTIONS}

\subsection{Unreleased Resonant Body Transistors}

One of the greatest challenges currently faced by NEMS is that of integration into modern CMOS technology. The majority of electromechanical devices require a release step to freely suspend the moving structures. This necessitates costly complex encapsulation methods and restricts NEMS fabrication to backend-of-line (BEOL) processing. However, as evidenced in Chapter 7, fabrication of Resonant Body Transistors (RBTs) is very similar to that of split-gate FinFETs. Design of an unreleased RBT at the transistor level of the CMOS stack would enable direct integration into front-end-of-line (FEOL) processing, making the RBT an attractive choice for on-chip signal generation. In the CMOS stack, an unreleased RBT can be comprised of a FinFET with gate geometry defining acoustic resonance, as described in Chapter 6. As in the case of the released RBT, elastic waves inside the FinFET peizoresistively modulate the drain current.

Realization of an unreleased mechanical resonator poses an interesting challenge of localizing acoustic waves in an inhomogeneous solid (i.e. the CMOS stack). 2D and 3D inhomogeneities can be designed to localize acoustic excitations in FinFETs, implementing impedance mismatch patterns using materials available in the CMOS stack to form an acoustic Bragg reflector surrounding a FinFET and focusing acoustic energy on the FinFET at a desired resonant frequency (Figure 9.1. Acoustic Bragg reflectors are commonly used for 1-dimensional localization in Film Bulk Acoustic Resonators (FBARs), but have not yet been developed in multi-dimensional systems. The Bragg reflectors will 
serve to enhance resonator $Q$ and isolate acoustic waves form non-resonant devices on chip, preventing unwanted parasitic cross-talk.

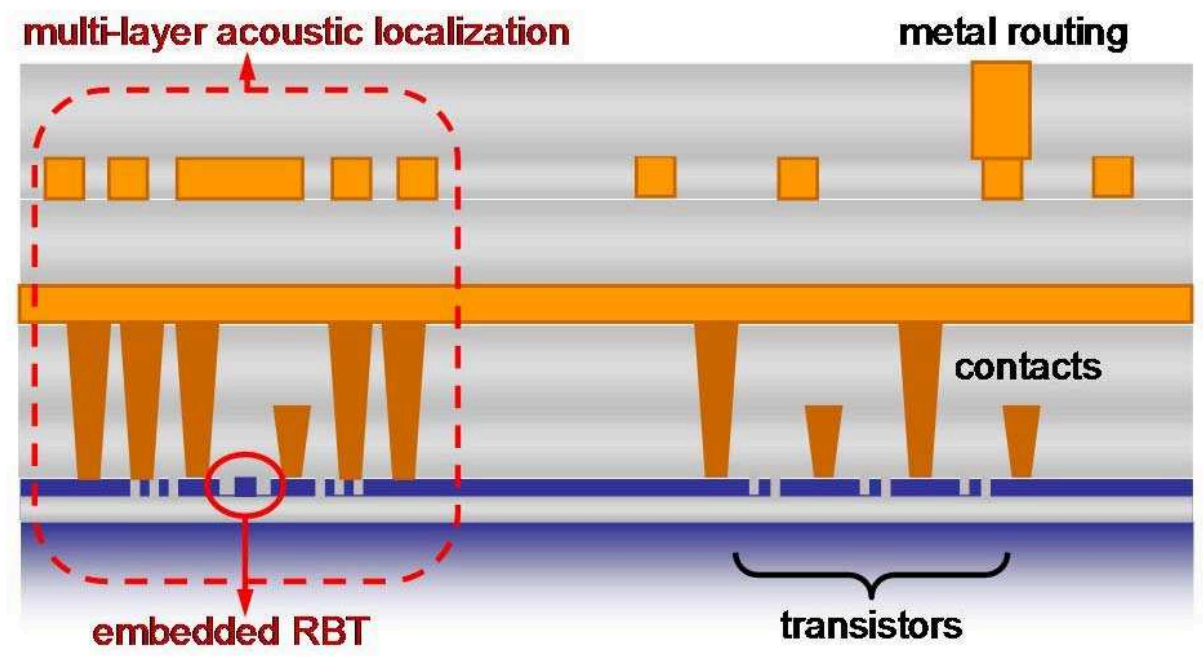

Figure 9.1: Cross section of CMOS stack showing acoustically excited Resonant Body Transistors and patterning of silicon, insulation, and metal layers to localize acoustic resonance.

\subsection{The Resonant Body Oscillator}

The RBT can be further implemented to form a Resonant Body Oscillator. The crystal and transistor of a generic Pierce oscillator can be replaced by a single RBT (Figure 9.2), which can be engineered to incorporate shunt capacitors. The entire Pierce crystal oscillator can therefore be formed from a single RBT, with a footprint of $<1 \mu \mathrm{m}^{2}$. The low phase noise of the oscillator due to the high $Q$ and 
high resonant frequency of the RBT will produce a high sensitivity, low power consumption RBO.
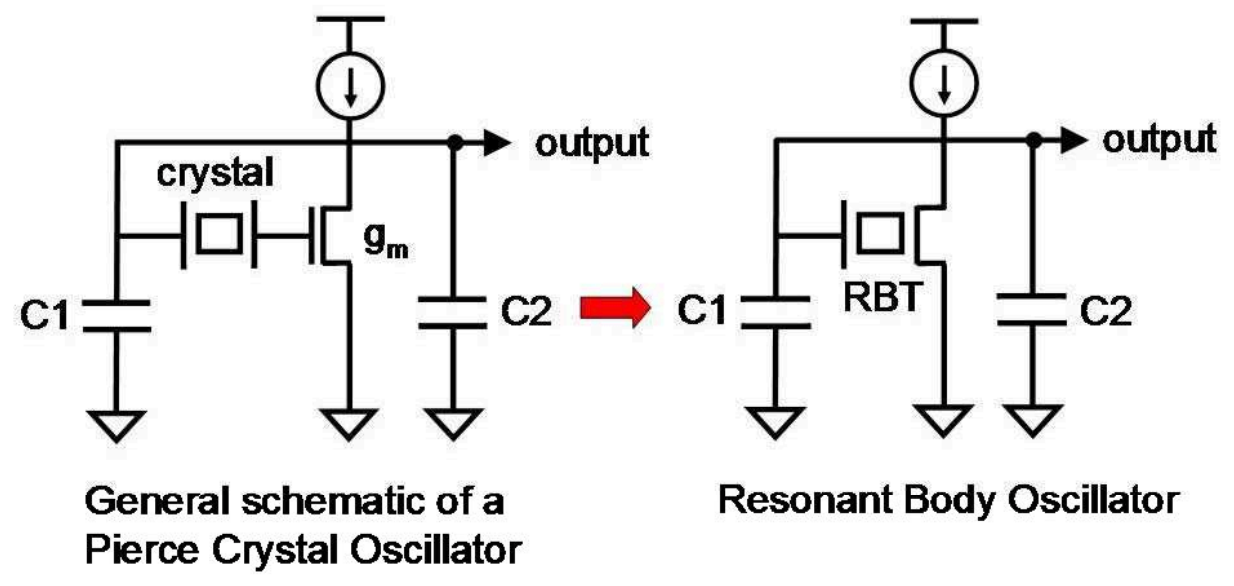

Figure 9.2: Schematic of Resonant Body Oscillator comprising an RBT. Acoustic resonance in the transistor will enable low-power, low phase noise oscillators at $\mathrm{GHz}$ frequencies with $<1 \mu \mathrm{m}^{2}$ footprint.

\subsection{Synchronization}

Synchronization of oscillators commonly involves injection locking using a tail current running through a series of oscillators. However, locking $N$ oscillator tanks in this configuration limits both headroom and output swing of each oscillator by a factor of $N$. Instead, acoustic injection locking through mechanical energy transfer in a large array of RBOs can circumvent the headroom and power 


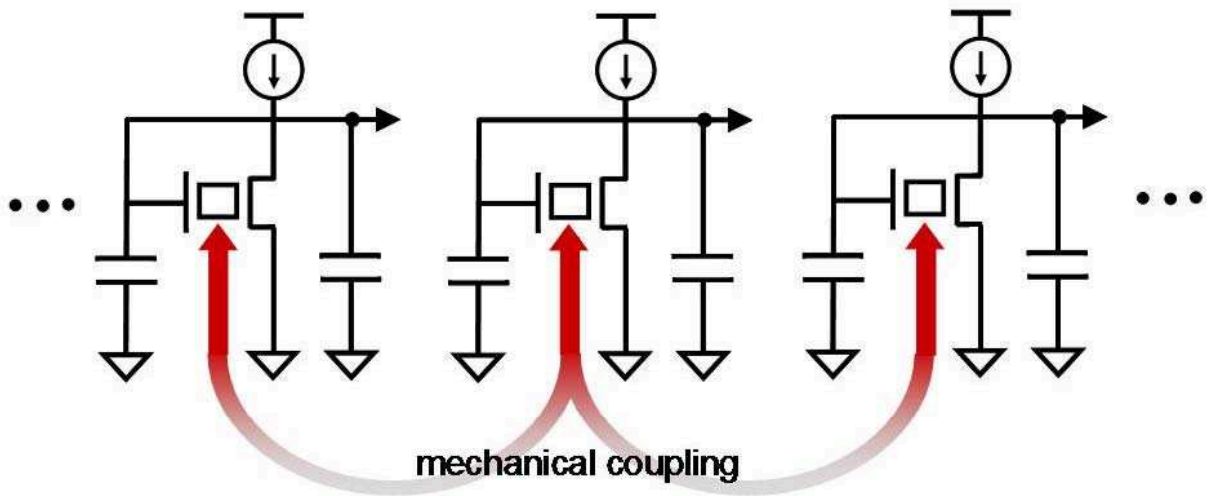

Figure 9.3: Acoustic synchronization of an array of Resonant Body Oscillators through mechanical coupling between adjacent Resonant Body Transistors. Coupling can be induced from vibrations through the substrate or insulation or using mechanical coupling springs.

consumption penalties of conventional electrically-based synchronization (Figure 9.3). Acoustically synchronized arrays of RBOs will enable low-power local clock generation, synchronized globally across a microprocessor. 


\section{APPENDIX A \\ DIELECTRIC VS. PIEZOELECTRIC TRANSDUCTION}

\section{A.1 Dielectric Film Actuation}

The dielectric film actuator is a thin deposited film of high permittivity dielectric material on one surface of a silicon cantilever beam. A conductive layer is evaporated onto this dielectric, and a voltage $V_{D C}+v_{i n}$ is applied between this electrode and the conductive silicon cantilever. The applied voltage creates an attractive force between the electrode and cantilever, squeezing the dielectric film. The film is then forced to squeeze outwards, causing a lateral stress in the beam, driving beam oscillations at the input frequency.

This actuator requires both driving and sensing electrodes. A simple dualelectrode design consists of two strips of metal running the length of the beam, each extending half the width of the beam. We assume that since $w \ll L$, the dielectric film stress is distributed over the entire width of the beam, such that the deflection is symmetric along the width of the beam.

\section{A.1.1 Beam Shape Due To Dielectric Stress Transfer}

The force between the electrode and beam is given by

$$
\begin{aligned}
F_{z} & =-\frac{\partial W}{\partial z} \\
& =-\frac{\partial}{\partial z}\left(\frac{1}{2} C V^{2}\right) \\
& =-\frac{1}{2} \frac{\partial C}{\partial z}\left(V_{D C}+v_{i n}\right)^{2}
\end{aligned}
$$




$$
=-V_{D C} v_{i n} \frac{\partial C}{\partial z}
$$

where the $V_{D C}^{2}$ term is neglected as a constant offset and the $v_{i n}^{2}$ term is a small, higher order term. The capacitance is just

$$
C=\frac{\epsilon_{0} \epsilon_{f} w L}{2 h_{f}}
$$

Here, $\epsilon_{f}$ and $h_{f}$ are the relative permittivity and thickness of the dielectric film, respectively. We can write the force as

$$
F_{z}=\frac{\epsilon_{0} \epsilon_{f} w L}{2 h_{f}^{2}}
$$

This capacitor plate force creates a vertical strain $\varepsilon_{z}$ in the dielectric, generating a lateral strain $\varepsilon_{x}=v_{f} \varepsilon_{z}$, where $v_{f}$ is the Poisson ratio of the dielectric. This strain corresponds to an effective residual stress in the film

$$
\begin{aligned}
\sigma_{x, f} & =\frac{E_{f}^{*} v_{f} F_{z}}{E_{f} w L} \\
& =\frac{\epsilon_{0} \epsilon_{f} v_{f} V_{D C} v_{i n}}{2\left(1-v_{f}\right) h_{f}^{2}} \\
& \equiv \sigma_{0}
\end{aligned}
$$

where $E_{f}^{*}$ is the biaxial Young's modulus of the film $\left(E^{*}=\frac{E}{1-\nu}\right)$. This stress generates a strain $\varepsilon_{a x}$ in the entire beam, causing the beam to bend. Then

$$
\begin{aligned}
\sigma_{0} h_{f} w & =E_{b}^{*} h_{b} w \varepsilon_{a x}+E_{f}^{*} h_{f} w \varepsilon_{a x} \\
\varepsilon_{a x} & =\frac{h_{f} \sigma_{0}}{E_{b}^{*} h_{b}+E_{f}^{*} h_{f}}
\end{aligned}
$$


where $h_{b}$ is the thickness of the beam and $E_{b}^{*}$ is the biaxial Young's modulus of the beam. In order to find the distributed stress in the film and beam, we impose the boundary condition that at every point $x$ along the length of the beam, the total force in the beam is equal to the total force in the film.

$$
\begin{aligned}
\sigma_{f} & =\frac{E_{b}^{*} h_{b} \sigma_{0}}{E_{b}^{*} h_{b}+E_{f}^{*} h_{f}} \\
\sigma_{f} & =\frac{E_{b}^{*} h_{f} \sigma_{0}}{E_{b}^{*} h_{b}+E_{f}^{*} h_{f}}
\end{aligned}
$$

We can now calculate the total moment about center of the beam as a function of the position $x$ along the beam. In the approximation $h_{f} \ll h_{b}$, the moment contribution from the film stress is taken at $h_{b} / 2$. In this limit, we can also approximate $\sigma_{f} \approx \sigma_{0}$, so that $\sigma_{b} \approx \frac{h_{f}}{h_{b}} \sigma_{0}$. Then

$$
\begin{aligned}
M & =M_{b}+M_{f} \\
& =\frac{1}{2} h_{b}^{2} w \sigma_{b}+\frac{1}{2} h_{b} h_{f} w \sigma_{f} \\
& \simeq \frac{1}{2} h_{b}^{2} w \sigma_{b} \\
& \simeq \frac{1}{2} h_{b} h_{f} w \sigma_{0}=\frac{h_{b} w \epsilon_{0} \epsilon_{f} v_{f} V_{D C} v_{i n}}{4 h_{f}\left(1-v_{f}\right)}
\end{aligned}
$$

The deflection $\mathrm{z}^{\prime \prime}(\mathrm{x})$ is

$$
z^{\prime \prime}(x)=\frac{-M}{E_{b}^{*} I_{b}}=\frac{-12 M}{E_{b}^{*} w h_{b}^{3}}
$$

Plugging Equation A.11 into Equation A.12, the vertical deflection of the beam is given by

$$
z(x)=Q \frac{3 v_{f} \epsilon_{0} \epsilon_{f}\left(1-v_{b}\right) V_{D C} v_{i n}}{2 E_{b} h_{f} h_{b}^{2}\left(1-v_{f}\right)} x^{2}
$$


so that the deflection at the tip is

$$
\delta_{\text {diel }}=Q \frac{3 v_{f} \epsilon_{0} \epsilon_{f}\left(1-v_{b}\right) V_{D C} v_{i n} L^{2}}{2 E_{b} h_{f} h_{b}^{2}\left(1-v_{f}\right)}
$$

\section{A.1.2 Sensing And Motional Impedance}

The sensing electrode covering the second half of the dielectric is used to measure the output current $i_{\text {out }}$ due to the input voltage $v_{i n}$. The motional impedance $R_{x, \text { diel }} \equiv v_{\text {in }} / i_{\text {out }}$ requires the calculation of the output current generated at the sensing electrode.

$$
i_{\text {out }}=\frac{d Q}{d t}=\frac{d}{d t} C\left(v_{\text {in }}+V_{D C}\right) \approx V_{D C} \frac{d C}{d t}
$$

The time derivative of the capacitance can be expanded into $\frac{d C}{d t}=\frac{d C}{d h} \frac{d h}{d t}$, where $h(t)$ is the thickness of the dielectric film. The strain induced in the beam due to deflection is a superposition of bending and extensional strain. In the case of small deflections, the extensional strain is ignored, and

$$
\varepsilon_{x}=\varepsilon_{x, \text { bend }}=\frac{1}{2} h_{b} z^{\prime \prime}(L)=\frac{h_{b} \delta_{\text {diel }}}{L^{2}}
$$

The bending strain in Equation A.16 is obtained from the fact that the vertical deflection of the beam $\delta_{z}$ is independent of $\mathrm{z}$, and that there is no vertical shear strain $\varepsilon_{x z}$. We take the longitudinal strain $\varepsilon_{x}$ to be dominant, so that $\varepsilon_{x}=\frac{1}{E}\left(\sigma_{x}-\right.$ $\left.v\left(\sigma_{y}+\sigma_{z}\right)\right) \approx \frac{1}{E} \sigma_{x}$ 
The vertical shear strain is given by

$$
\varepsilon_{x z}=\frac{1}{2}\left(\frac{\partial \delta_{x}}{\partial z}+\frac{\partial \delta_{z}}{\partial x}\right)=0
$$

Then

$$
\frac{\partial \delta_{x}}{\partial z}=-\frac{\partial \delta_{z}}{\partial x}
$$

We can then write the longitudinal deflection $\delta_{x}$ as

$$
\delta_{x}=-\int \frac{d \delta_{z}(x)}{d x} d z=-z \frac{d \delta_{z}}{d x}
$$

so that the longitudinal strain is

$$
\varepsilon_{x}=\frac{\partial \delta_{x}}{\partial x}=-z \frac{d^{2} \delta_{z}}{d x^{2}}
$$

Therefore, the strain at the dielectric film is given by

$$
\varepsilon_{x}=\frac{h_{b}}{2} \frac{d^{2} \delta_{z}}{d x^{2}}
$$

which is equivalent to Equation A.16. Taking the biaxial modulus into account,the stress induced in the film is then

$$
\sigma_{x, f}=\frac{E_{f}}{\left(1-v_{f}\right)} \varepsilon_{x}
$$

so that the induced vertical strain is

$$
\varepsilon_{z, f}=\frac{v_{f} \sigma_{x, f}}{E_{f}\left(1-v_{f}\right)}
$$


We can therefore determine the time derivative of the film thickness:

$$
\frac{d}{d t}\left(h_{f}+\Delta h\right)=\frac{d \Delta h}{d t}=\frac{d}{d t}\left(h_{f} \varepsilon_{x, f}\right)=\frac{\omega v_{f} h_{f} h_{b} \delta_{\text {diel }}}{\left(1-v_{f}\right) L^{2}}
$$

The time derivative of the capacitance is then

$$
\begin{aligned}
\frac{d C}{d t} & =\frac{d C}{d h} \frac{d h}{d t} \\
& =\frac{\epsilon_{0} \epsilon_{f} w L}{2 h_{f}^{2}} \frac{\omega v_{f} h_{f} h_{b} \delta_{\text {diel }}}{\left(1-v_{f}\right) L^{2}}
\end{aligned}
$$

Again, the factor of $1 / 2$ in the capacitance term is due to the fact that the width of the sensing electrode is only one half the full width of the cantilever. The transfer function in Equation A.15 can then be written as

$$
i_{\text {out }}=\frac{\omega \epsilon_{0} \epsilon_{f} v_{f}}{2\left(1-v_{f}\right)} \frac{w}{L} \frac{h_{b}}{h_{f}} \delta_{\text {diel }} V_{D C}
$$

and the motional resistance is

$$
\begin{aligned}
R_{x, \text { diel }} & =\frac{v_{\text {in }}}{i_{\text {out }}} \\
& =\frac{2\left(1-v_{f}\right) L h_{f} v_{\text {in }}}{Q \omega \epsilon_{0} \epsilon_{f} v_{f} w h_{b} \delta_{\text {diel }} V_{D C}} \\
& =\frac{4\left(1-v_{f}\right) E_{b} h_{f}^{2} h_{b}}{3 Q \omega\left(\epsilon_{0} \epsilon_{f} v_{f}\right)^{2}\left(1-v_{b}\right) w L V_{D C}^{2}}
\end{aligned}
$$




\section{A.2 Piezoelectric Film Actuation}

The piezoelectric film actuation geometry is identical to that of the dielectric. A piezoelectric material is sandwiched between a top electrode and the silicon cantilever. A voltage $v_{i n}$ applied to the piezoelectric film generates a lateral strain in the film, causing the beam to bend.

\section{A.2.1 Beam Shape Due To Piezoelectric Stress Transfer}

As with the dielectric actuation case, we consider two electrodes running the full length of the cantilever, each spanning half the width. One electrode is used for actuation, while the other is reserved for sensing. The lateral strain $\varepsilon_{x}$ due to an applied voltage $v_{i n}$ is

$$
\varepsilon_{x}=d_{31} E_{z}=d_{31} \frac{v_{i n}}{h_{f}}
$$

where $h_{f}$ is again the film thickness, and $d_{31}$ is the piezoelectric stress coefficient coupling the $\mathrm{z}$ and $\mathrm{x}$ axes. For a piezoelectric Young's modulus $E_{p}$, the stress in the film is

$$
\sigma_{x}=E_{p} \varepsilon_{x}=d_{31} E_{z}=d_{31} E_{p} \frac{v_{i n}}{h_{f}}
$$

The moment about the central axis can now be calculated. Again, assuming $h_{f} \ll h_{b}$, the moment can be approximated to

$$
M(x)=\int_{0}^{h_{f}} \sigma_{x} \frac{w}{2} \frac{h_{b}}{2} d z=\frac{1}{4} d_{31} E_{p} v_{i n} w h_{b}
$$


Here, the factor of two in the width comes from the fact that a field is only being applied The deflection of the beam can then be determined:

$$
z^{\prime \prime}(x)=\frac{-M}{E_{b}^{*} I_{b}}=\frac{-12 M}{E_{b}^{*} w h_{b}^{3}}
$$

Integrating twice, the displacement of the cantilever is

$$
z(x)=-\frac{3 d_{31} E_{p} v_{i n}}{2 E_{b} h_{b}^{2}} x^{2}
$$

\section{A.2.2 Sensing And Motional Impedance}

The beam bending described by Equation A.32 results in a lateral stress in the piezoelectric film. The stress can be thought of as the superposition of bending stress $\sigma_{x, b e n d}$ and extensional stress $\sigma_{x, e x t}$.

$$
\begin{aligned}
\sigma_{x} & =\sigma_{x, \text { bend }}+\sigma_{x, \text { ext }} \\
& =E_{b}\left(-\frac{h_{b} z^{\prime \prime}}{2}+\frac{1}{2 L} \int_{0}^{L}\left(z^{\prime}(x)\right)^{2} d x\right) \\
& =E_{b}\left(\frac{3 d_{31} E_{p} v_{\text {in }}}{2 E_{b} h_{b}}+\left(\frac{3 d_{31} E_{p} v_{\text {in }}}{2 E_{b} h_{b}^{2}}\right)^{2} \frac{L^{2}}{6}\right)
\end{aligned}
$$

For small deflections, the extensional term in the stress is negligible, though it is important to note its small nonlinear contribution to the transfer function. The displacement current induced in the piezoelectric film can be calculated directly from the computed stress.

$$
D_{3}=d_{31} \sigma_{x}
$$


Then the output current can be obtained:

$$
\begin{aligned}
i_{\text {out }} & =\omega \int_{0}^{L} \int_{0}^{w / 2} D_{3} d y d x \\
& =\frac{3 Q \omega d_{31}^{2} E_{p}^{2} w L v_{\text {in }}}{4 E_{b} h_{b}}
\end{aligned}
$$

The quality factor $Q$ is inserted to account for power dissipated in the system. Equation A.34 gives the transfer function for the piezoelectric film actuator. We can therefore extract the motional impedance $R_{x, \text { piezo }}$

$$
R_{x, \text { piezo }} \equiv \frac{v_{\text {in }}}{i_{\text {out }}}=\frac{4 E_{b} h_{b}}{3 Q \omega d_{31}^{2} E_{p}^{2} w L}
$$

\section{A.3 Comparison Of Actuation Transfer Functions}

Reducing the motional impedance of a MEMS device is critical to its integration into a larger circuit. It is therefore useful to compare the motional impedance of dielectric and piezoelectric film actuators. From Equations A.27 and A.35, the ratio of motional impedance for the two actuation methods is

$$
\frac{R_{x, \text { diel }}}{R_{x, \text { peizo }}}=\frac{Q_{\text {piezo }}}{Q_{\text {diel }}} \frac{\left(1-v_{f}\right)}{\left(1-v_{b}\right)}\left(\frac{E_{p} d_{31}}{\epsilon_{0} \epsilon_{f} v_{f}}\right)^{2} \frac{1}{\left(V_{D C} / h_{f}\right)^{2}}
$$

Equation A.36 reveals several important things. Because dielectrics are easier and cheaper to manufacture, a small ratio for Equation A.36 is desired. This indicates that a high-K dielectric material with a high Poisson ratio $v_{f}$ is needed. The dielectric must have a high breakdown field $V_{D C} / h_{f}$. If these material properties are not sufficient, piezoelectric actuation should be used. One should note 
the lack of dependence on the absolute dimensions of the cantilever. 


\section{APPENDIX B}

\section{RBT FABRICATION PROCESS}

Provided below is the comprehensive list of steps required to fabricate the nFET Resonant Body Transistor. This process is optimized for use in the Cornell Nanoscale Science and Technology Facility, but may be transferred to other facilities.

Table B.1: RBT Fabrication Process

\begin{tabular}{|c|c|c|c|c|}
\hline Gate & ProcType & Mat/Mask & Tool & Process \\
\hline $\mathrm{ALN}$ & Start & SOI & SOITEC & $\begin{array}{l}\text { Starting SOI wafer, } 400 \mathrm{~nm} \\
\text { BOX, } 340 \mathrm{~nm} \text { lightly doped p- } \\
\text { type device layer }\end{array}$ \\
\hline ALN & Clean & RCA & MOS & MOS clean with HF dip \\
\hline$\overline{\mathrm{ALN}}$ & Growth & $\mathrm{SiO}_{2}$ & Furnace & $\begin{array}{l}\text { Dry HCL thermal oxide } \\
\text { growth }\left(1100^{\circ} \mathrm{C}, 120 \mathrm{~min}\right)\end{array}$ \\
\hline ALN & Spin & PMMA & EBSpin & $\begin{array}{l}\text { Spin } 2 \mu m \text { E-Beam resist } \\
(495 \text { PMMA } 11 \% \text { Anisole, } \\
\text { 1000rpm, } 60 \mathrm{sec})\end{array}$ \\
\hline ALN & Bake & PMMA & HotPlate & Pre-Bake $\left(170^{\circ} \mathrm{C}, 15 \mathrm{~min}\right)$ \\
\hline ALN & Expose & ALN & JEOL9300 & $5 \mathrm{nA}, 100 \mathrm{keV}, 2200 \mu \mathrm{C} / \mathrm{cm}^{2}$ \\
\hline$\overline{\mathrm{ALN}}$ & Develop & MIBK & Wet & $\begin{array}{l}\text { Develop in MIBK:IPA 1:3, } \\
\text { 75sec }\end{array}$ \\
\hline ALN & Rinse & $\mathrm{DI} \mathrm{H}_{2} \mathrm{O}$ & Wet & Rinse in DI Water \\
\hline ALN & Dry & $\mathrm{N}_{2}$ & $\mathrm{~N}_{2}$ Gun & Blow dry \\
\hline$\overline{A L N}$ & Descum & PMMA & $\mathrm{Ox80}$ & $\begin{array}{l}\text { ALN resist descum (30mT, } \\
\left.100 \mathrm{~W}, 50 \mathrm{sccm} \mathrm{O}_{2}, 90 \mathrm{sec}\right)\end{array}$ \\
\hline ALN & Etch & $\overline{\mathrm{SiO}_{2}}$ & Ox100 & $\begin{array}{l}\text { ALN etch of HOM (100sccm } \\
\mathrm{CHF}_{3}, 2 \mathrm{sccm} \mathrm{O} \mathrm{O}_{2}, 15 \mathrm{~W} \text { RF, } \\
\left.2500 \mathrm{~W} \text { ICP, } 5 \mathrm{mTorr}, 11^{\circ} \mathrm{C}\right)\end{array}$ \\
\hline $\mathrm{ALN}$ & Etch & Si & Unaxis & $\begin{array}{l}\text { ALN etch of device layer } \\
\text { (0Trench recipe, } 3 \text { loops) }\end{array}$ \\
\hline ALN & Etch & $\mathrm{SiO}_{2}$ & Ox100 & $\begin{array}{l}\text { ALN etch of BOX } 100 \mathrm{sccm} \\
\mathrm{CHF}_{3}, 2 \mathrm{sccm} \mathrm{O} \mathrm{O}_{2}, 15 \mathrm{~W} \text { RF, } \\
\left.2500 \mathrm{~W} \text { ICP }, 5 \mathrm{mTorr}, 11^{\circ} \mathrm{C}\right)\end{array}$ \\
\hline$\overline{\mathrm{ALN}}$ & Strip & PMMA & Wet & $\begin{array}{l}\text { Strip PMMA } \\
\text { Chloride with } \\
\text { tion) }\end{array}$ \\
\hline
\end{tabular}

Continued on Next Page... 
Table B.1 - Continued

\begin{tabular}{|c|c|c|c|c|}
\hline Gate & ProcType & Mat/Mask & Tool & Process \\
\hline$\overline{\mathrm{ALN}}$ & Rinse & IPA & Wet & Rinse in IPA \\
\hline ALN & Dry & $\mathrm{N}_{2}$ & $\mathrm{~N}_{2}$ Gun & Blow dry \\
\hline ALN & Clean & $\mathrm{O}_{2}$ & Aura & $\mathrm{O}_{2}$ plasma clean (recipe $2 \times 2$ ) \\
\hline ALN & Clean & Hot Pir & Hamatech & Hot Piranha clean \\
\hline ALN & Etch & $\mathrm{Si}$ & PT770 & $\begin{array}{l}\text { ALN Cl etch of handle wafer } \\
(700 \mathrm{~nm})\end{array}$ \\
\hline ALN & Clean & $\mathrm{O}_{2}$ & Aura & $\mathrm{O}_{2}$ plasma clean (recipe $2 \times 4$ ) \\
\hline ALN & Clean & Hot Pir & Hamatech & Hot Piranha clean \\
\hline \multicolumn{5}{|c|}{ RX Gate } \\
\hline RX & Spin & ma-N & EBSpin & 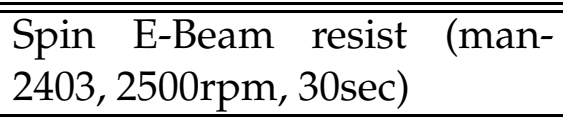 \\
\hline RX & Bake & ma-N & HotPlate & Pre-Bake $\left(90^{\circ} \mathrm{C}, 60 \mathrm{sec}\right)$ \\
\hline RX & Expose & RX & JEOL9300 & $1 \mathrm{nA}, 100 \mathrm{keV}, 1100 \mu \mathrm{C} / \mathrm{cm}^{2}$ \\
\hline $\mathrm{RX}$ & Develop & MIF300 & Wet & Develop in MIF-300, 75sec \\
\hline $\mathrm{RX}$ & Rinse & $\mathrm{DI} \mathrm{H}_{2} \mathrm{O}$ & Wet & Rinse in DI Water \\
\hline RX & Dry & $\mathrm{N}_{2}$ & $\mathrm{~N}_{2}$ Gun & Blow dry \\
\hline $\mathrm{RX}$ & Etch & $\mathrm{SiO}_{2}$ & Ox100 & $\begin{array}{l}\text { RX etch of } \mathrm{HOM}(100 \mathrm{sccm} \\
\mathrm{CHF}_{3}, 2 \mathrm{sccm} \mathrm{O} \mathrm{O}_{2}, 15 \mathrm{~W} \mathrm{RF}, \\
\left.2500 \mathrm{~W} \mathrm{ICP}, 5 \mathrm{mTorr}^{\circ} 11^{\circ} \mathrm{C}\right)\end{array}$ \\
\hline $\mathrm{RX}$ & Clean & $\mathrm{O}_{2}$ & Aura & $\mathrm{O}_{2}$ plasma clean (recipe $2 \times 2$ ) \\
\hline $\mathrm{RX}$ & Clean & Hot Pir & Hamatech & Hot Piranha clean \\
\hline RX & Etch & $\mathrm{Si}$ & PT770 & $\mathrm{RXCl}$ etch of device layer \\
\hline RX & Clean & $\mathrm{O}_{2}$ & Aura & $\mathrm{O}_{2}$ plasma clean (recipe $2 \times 2$ ) \\
\hline RX & Clean & Hot Pir & Hamatech & Hot Piranha clean \\
\hline RX & Clean & RCA & MOS & MOS clean \\
\hline $\mathrm{RX}$ & Growth & $\mathrm{SiO}_{2}$ & Furnace & $\begin{array}{l}\text { Dry HCL sacrificial oxidation } \\
\left(900^{\circ} \mathrm{C}, 3 \mathrm{~min}\right)\end{array}$ \\
\hline $\mathrm{RX}$ & Etch & $\mathrm{SiO}_{2}$ & Wet & $\begin{array}{l}\text { HF } 100: 1 \text { strip of sidewall ox- } \\
\text { ide, } 45 \mathrm{sec}\end{array}$ \\
\hline
\end{tabular}

PC Gate

\begin{tabular}{|c|c|c|c|l|}
\hline \hline PC & Clean & RCA & MOS & MOS clean \\
\hline PC & Dep & SiN & Furnace & $\begin{array}{l}15 \mathrm{~nm} \text { LPCVD Std. Nitride } \\
\text { deposition }\left(775^{\circ} \mathrm{C}, 4 \mathrm{~min}\right)\end{array}$ \\
\hline PC & Dep & n+Poly & Furnace & $\begin{array}{l}430 \mathrm{~nm} \text { LPCVD n+ Poly depo- } \\
\text { sition }\left(590^{\circ} \mathrm{C}, 430 \mathrm{~min}\right)\end{array}$ \\
\hline PC & Anneal & $\mathrm{n}+$ Poly & Furnace & $\mathrm{N}_{2}$ anneal $\left(1050^{\circ} \mathrm{C}, 1 \mathrm{hr}\right)$ \\
\hline
\end{tabular}

Continued on Next Page... 
Table B.1 - Continued

\begin{tabular}{|c|c|c|c|c|}
\hline Gate & ProcType & Mat/Mask & Tool & Process \\
\hline PC & Etch & $\mathrm{SiO}_{2}$ & Wet & $\begin{array}{l}\text { HF 100:1 strip of native oxide, } \\
20 \mathrm{sec}\end{array}$ \\
\hline PC & CMP & n+Poly & Strasbaugh & $\begin{array}{lll}\text { Poly planarization } & \text { (IT1400 } \\
\text { Pad, P1000 slurry, } & 7.5 p s i, \\
15 \mathrm{sec}) & & \end{array}$ \\
\hline PC & Clean & n+Poly & Wet & $\begin{array}{l}\text { Post-CMP clean (Std. Clean 1, } \\
\text { ultrasonication, } 10 \mathrm{~min} \text { ) }\end{array}$ \\
\hline PC & Clean & $\mathrm{n}+$ Poly & Hamatech & $\begin{array}{l}\mathrm{NH}_{4} \mathrm{OH} / \mathrm{H}_{2} \mathrm{O}_{2} \text { Hamatech } \\
\text { front-side clean }\end{array}$ \\
\hline PC & Clean & RCA & MOS & ith HF dip 20sec \\
\hline PC & Dep & $\mathrm{SiO}_{2}$ & GSI & $\begin{array}{l}\text { PECVD N1.46 oxide deposi- } \\
\text { tion }\left(400^{\circ} \mathrm{C}, 80 \mathrm{sec}\right)\end{array}$ \\
\hline PC & Clean & RCA & MOS & MOS clean \\
\hline $\mathrm{PC}$ & Anneal & $\mathrm{SiO}_{2}$ & Furnace & $\begin{array}{l}\mathrm{N}_{2} \text { anneal }\left(400-800^{\circ} \mathrm{C} \text { ramp }\right. \\
\left.2 \mathrm{hr}, 800^{\circ} \mathrm{C} 45 \mathrm{~min}\right)\end{array}$ \\
\hline PC & Spin & ma-N & EBSpin & $\begin{array}{l}\text { Spin E-Beam resist (man- } \\
2403,2500 \mathrm{rpm}, 30 \mathrm{sec})\end{array}$ \\
\hline PC & Bake & ma-N & HotPlate & Pre-Bake $\left(90^{\circ} \mathrm{C}, 60 \mathrm{sec}\right)$ \\
\hline PC & Expose & PC & JEOL9300 & $1 \mathrm{nA}, 100 \mathrm{keV}, 1000 \mu \mathrm{C} / \mathrm{cm}^{2}$ \\
\hline PC & Develop & MIF300 & Wet & Develop in MIF-300, 75sec \\
\hline PC & Rinse & $\mathrm{DI} \mathrm{H}_{2} \mathrm{O}$ & Wet & Rinse in DI Water \\
\hline PC & Dry & $\mathrm{N}_{2}$ & $\mathrm{~N}_{2}$ Gun & Blow dry \\
\hline PC & Etch & $\mathrm{SiO}_{2}$ & Ox100 & $\begin{array}{l}\text { PC etch of } \mathrm{HOM}(90 \mathrm{sccm} \\
\mathrm{CHF}_{3}, 5 \mathrm{sccm} \mathrm{O}_{2}, 10 \mathrm{sccm} \mathrm{CF}_{4} \text {, } \\
15 \mathrm{~W} \mathrm{RF}, 2500 \mathrm{~W} \mathrm{ICP}, 5 \mathrm{mTorr}^{2} \\
\left.11^{\circ} \mathrm{C}\right)\end{array}$ \\
\hline PC & ean & $\mathrm{O}_{2}$ & Aura & $\mathrm{O}_{2}$ plasma clean (recipe $2 \times 2$ ) \\
\hline PC & Clean & Hot Pir & Hamatech & Hot Piranha clean \\
\hline PC & $\mathrm{ch}$ & $\mathrm{n}+$ Poly & PT770 & PC Cl etch of polysilicon \\
\hline PC & Clean & $\mathrm{O}_{2}$ & Aura & $\mathrm{O}_{2}$ plasma clean (recipe $2 \times 2$ ) \\
\hline PC & Clean & Hot Pir & Hamatech & Hot Piranha clean \\
\hline
\end{tabular}

Ion Implant

\begin{tabular}{|c|c|c|c|l|}
\hline \hline IMP & Etch & $\mathrm{SiO}_{2}$ & Ox80 & $\begin{array}{l}\text { Blanket oxide etch to re- } \\
\text { move } \mathrm{RX} \mathrm{HOM}(40 \mathrm{mT}, 240 \mathrm{~W}, \\
\left.50 \text { sccm } \mathrm{CHF}_{3}, 2 \mathrm{sccm} \mathrm{\textrm {O } _ { 2 }}\right)\end{array}$ \\
\hline IMP & Clean & $\mathrm{O}_{2}$ & Aura & $\mathrm{O}_{2}$ plasma clean (recipe 2 2 2) \\
\hline IMP & Clean & Hot Pir & Hamatech & Hot Piranha clean \\
\hline
\end{tabular}

Continued on Next Page... 
Table B.1 - Continued

\begin{tabular}{|c|c|c|c|c|}
\hline Gate & ProcType & Mat/Mask & Tool & Process \\
\hline IMP & Implant & As & CoreSys & $\begin{array}{l}\text { As implant }\left(1 \mathrm{e} 15 \mathrm{~cm}^{2}, 70 \mathrm{keV},\right. \\
10^{o} \text { tilt, continuous rotation) }\end{array}$ \\
\hline$\overline{\mathrm{IMP}}$ & Clean & RCA & MOS & MOS clean \\
\hline IMP & Anneal & As & Furnace & $\mathrm{N}_{2}$ Anneal $\left(1000^{\circ} \mathrm{C}, 30 \mathrm{~min}\right)$ \\
\hline \multicolumn{5}{|c|}{ FOX } \\
\hline$\overline{\overline{\text { FOX }}}$ & Dep & $\mathrm{SiO}_{2}$ & GSI & $\begin{array}{l}\text { PECVD N1.46 oxide deposi- } \\
\text { tion }\left(400^{\circ} \mathrm{C}, 5 \mathrm{~min}, 1.1 \mu \mathrm{m}\right)\end{array}$ \\
\hline FOX & Spin & P20 & PhotoSpin & $\begin{array}{l}\text { Spin adhesion coat (P20, } \\
\text { 4000rpm, 30sec) }\end{array}$ \\
\hline FOX & Spin & SPR220 & PhotoSpin & $\begin{array}{l}\text { Spin photoresist (SPR220-3.0, } \\
\text { 4000rpm, 60sec) }\end{array}$ \\
\hline FOX & Bake & SPR220 & HotPlate & Pre-Bake $\left(115^{\circ} \mathrm{C}, 90 \mathrm{sec}\right)$ \\
\hline FOX & Expose & FOX & AS200 & FOX exposure $\left(40 \mathrm{~mJ} / \mathrm{cm}^{2}\right)$ \\
\hline$\overline{F O X}$ & Bake & SPR220 & HotPlate & $\begin{array}{l}\text { Post-exposure bake }\left(115^{\circ} \mathrm{C} \text {, }\right. \\
\text { 90sec) }\end{array}$ \\
\hline FOX & Develop & MIF300 & Hamatech & $\begin{array}{l}\text { Develop in MIF-300, 120sec } \\
\text { DP }\end{array}$ \\
\hline FOX & Descum & SPR220 & Ox80 & $\begin{array}{l}\text { FOX resist descum (30mT, } \\
\left.100 \mathrm{~W}, 50 \mathrm{sccm} \mathrm{O}_{2}, 2 \mathrm{~min}\right)\end{array}$ \\
\hline FOX & Etch & $\mathrm{SiO}_{2}$ & Ox80 & $\begin{array}{l}\text { FOX etch }(40 \mathrm{mT}, \quad 240 \mathrm{~W}, \\
\left.\left.50 \mathrm{sccm} \mathrm{CHF}_{3}, 2 \mathrm{sccm} \mathrm{O}\right)_{2}\right)\end{array}$ \\
\hline FOX & Clean & $\mathrm{O}_{2}$ & Aura & $\mathrm{O}_{2}$ plasma clean (recipe $2 \times 2$ ) \\
\hline FOX & Clean & Hot Pir & Hamatech & Hot Piranha clean \\
\hline \multicolumn{5}{|c|}{ M1 } \\
\hline M1 & Spin & $\mathrm{P} 20$ & PhotoSpin & $\begin{array}{l}\text { Spin adhesion coat (P20, } \\
\text { 4000rpm, 30sec) }\end{array}$ \\
\hline M1 & Spin & SPR220 & PhotoSpin & $\begin{array}{l}\text { Spin photoresist (SPR220-3.0, } \\
\text { 4000rpm, 60sec) }\end{array}$ \\
\hline M1 & Bake & SPR220 & HotPlate & Pre-Bake $\left(115^{\circ} \mathrm{C}, 90 \mathrm{sec}\right)$ \\
\hline M1 & Expose & M1 & AS200 & FOX exposure $\left(65 \mathrm{~mJ} / \mathrm{cm}^{2}\right)$ \\
\hline M1 & ImRev & SPR220 & YES & YES image reversal \\
\hline M1 & Expose & SPR220 & $\overline{\mathrm{ABM}}$ & Flood exposure (DUV, 60sec) \\
\hline M1 & Develop & MF321 & Wet & Develop in MF-321, 70sec \\
\hline M1 & Rinse & $\mathrm{DI} \mathrm{H}_{2} \mathrm{O}$ & Wet & Rinse in DI Water \\
\hline M1 & Dry & $\mathrm{N}_{2}$ & $\mathrm{~N}_{2}$ Gun & Blow dry \\
\hline M1 & Descum & SPR220 & Ox80 & $\begin{array}{l}\text { M1 resist descum (30mT, } \\
\left.100 \mathrm{~W}, 50 \mathrm{sccm} \mathrm{O}_{2}, 90 \mathrm{sec}\right)\end{array}$ \\
\hline
\end{tabular}

Continued on Next Page... 
Table B.1 - Continued

\begin{tabular}{|c|c|c|c|c|}
\hline Gate & ProcType & Mat/Mask & Tool & Process \\
\hline M1 & Etch & $\overline{\mathrm{SiO}_{2}}$ & Wet & $\begin{array}{l}\text { BOE 30:1 strip of native ox- } \\
\text { ide, 30sec }\end{array}$ \\
\hline M1 & Dep & $\mathrm{Ni}$ & CHA50 & $\begin{array}{l}\text { Conformal Ni e-beam evapo- } \\
\text { ration }(0.8 \AA / \mathrm{sec}, 40 \mathrm{~nm})\end{array}$ \\
\hline M1 & Liftoff & $\mathrm{Ni}$ & Wet & Lift-off in acetone \\
\hline M1 & Rinse & $\mathrm{Ni}$ & Wet & IPA rinse \\
\hline M1 & Dry & $\overline{\mathrm{N}_{2}}$ & $\mathrm{~N}_{2}$ Gun & Blow dry \\
\hline M1 & RTA & $\mathrm{Ni}$ & AG410 & Ni silicidation $\left(500^{\circ} \mathrm{C}, 30 \mathrm{sec}\right)$ \\
\hline M1 & Strip & Hot Pir & Hamatech & Hot Piranha strip of excess Ni \\
\hline M1 & Spin & P20 & PhotoSpin & $\begin{array}{l}\text { Spin adhesion coat (P20, } \\
\text { 4000rpm, 30sec) }\end{array}$ \\
\hline M1 & Spin & SPR220 & PhotoSpin & $\begin{array}{l}\text { Spin photoresist (SPR220-3.0, } \\
\text { 4000rpm, 60sec) }\end{array}$ \\
\hline M1 & Bake & SPR220 & HotPlate & Pre-Bake $\left(115^{\circ} \mathrm{C}, 90 \mathrm{sec}\right)$ \\
\hline M1 & Expose & M1 & AS200 & FOX exposure $\left(65 \mathrm{~mJ} / \mathrm{cm}^{2}\right)$ \\
\hline M1 & ImRev & SPR220 & YES & YES image reversal \\
\hline M1 & Expose & SPR220 & $\overline{\mathrm{ABM}}$ & Flood exposure (DUV, 60sec) \\
\hline M1 & Develop & MF321 & Wet & Develop in MF-321,70sec \\
\hline M1 & Rinse & DI $\mathrm{H}_{2} \mathrm{O}$ & Wet & Rinse in DI Water \\
\hline M1 & Dry & $\mathrm{N}_{2}$ & $\mathrm{~N}_{2}$ Gun & Blow dry \\
\hline M1 & Descum & SPR220 & Ox80 & $\begin{array}{l}\text { M1 resist descum }(30 \mathrm{mT} \text {, } \\
\left.100 \mathrm{~W}, 50 \mathrm{sccm} \mathrm{O}_{2}, 90 \mathrm{sec}\right)\end{array}$ \\
\hline M1 & Dep & $\mathrm{Ni}$ & CHA50 & $\begin{array}{l}\text { Conformal Ni e-beam evapo- } \\
\text { ration }(0.8 \AA / \mathrm{sec}, 120 \mathrm{~nm})\end{array}$ \\
\hline M1 & Liftoff & $\mathrm{Ni}$ & Wet & Lift-off in acetone \\
\hline M1 & Rinse & $\mathrm{Ni}$ & Wet & IPA rinse \\
\hline M1 & Dry & $\mathrm{N}_{2}$ & $\mathrm{~N}_{2}$ Gun & Blow dry \\
\hline
\end{tabular}

REL

\begin{tabular}{|c|c|c|c|l|}
\hline \hline REL & Spin & P20 & PhotoSpin & $\begin{array}{l}\text { Spin adhesion coat (P20, } \\
\text { 4000rpm, 30sec) }\end{array}$ \\
\hline REL & Spin & SPR220 & PhotoSpin & $\begin{array}{l}\text { Spin photoresist (SPR220-3.0, } \\
\text { 4000rpm, 60sec) }\end{array}$ \\
\hline REL & Bake & SPR220 & HotPlate & Pre-Bake $\left(115^{\circ} \mathrm{C}, 90 \mathrm{sec}\right)$ \\
\hline REL & Expose & REL & AS200 & REL exposure $\left(40 \mathrm{~mJ} / \mathrm{cm}^{2}\right)$ \\
\hline REL & Bake & SPR220 & HotPlate & $\begin{array}{l}\text { Post-exposure bake }\left(115^{\circ} \mathrm{C},\right. \\
\text { 90sec }\end{array}$ \\
\hline REL & Develop & MIF300 & Hamatech & $\begin{array}{l}\text { Develop in MIF-300, 120sec } \\
\text { DP }\end{array}$ \\
\hline
\end{tabular}

Continued on Next Page... 
Table B.1 - Continued

\begin{tabular}{|c|c|c|c|c|}
\hline Gate & ProcType & Mat/Mask & Tool & Process \\
\hline REL & Descum & SPR220 & Ox80 & $\begin{array}{l}\text { REL resist descum (30mT, } \\
\left.100 \mathrm{~W}, 50 \mathrm{sccm} \mathrm{O}_{2}, 2 \mathrm{~min}\right)\end{array}$ \\
\hline REL & Etch & $\mathrm{SiO}_{2}$ & Ox80 & $\begin{array}{l}\text { REL etch }(40 \mathrm{mT}, \quad 240 \mathrm{~W}, \\
\text { 50sccm } \mathrm{CHF}_{3}, \quad 2 \mathrm{sccm} \quad \mathrm{O}_{2}, \\
\text { 30min) }\end{array}$ \\
\hline REL & Strip & SPR220 & Ox80 & $\begin{array}{l}\text { REL resist strip (30mT, 100W, } \\
\left.50 \mathrm{sccm} \mathrm{O}_{2}, 6 \mathrm{~min}\right)\end{array}$ \\
\hline REL & Strip & SPR220 & Wet & $\begin{array}{l}\text { Strip remaining SPR220-3.0 in } \\
\text { acetone }\end{array}$ \\
\hline REL & Rinse & SPR220 & Wet & IPA $\mathrm{r}$ \\
\hline REL & Dry & $\mathrm{N}_{2}$ & $\mathrm{~N}_{2}$ Gun & Blow dry \\
\hline REL & Spin & P20 & PhotoSpin & $\begin{array}{l}\text { Spin adhesion coat (P20, } \\
\text { 4000rpm, 30sec) }\end{array}$ \\
\hline REL & Spin & SPR220 & PhotoSpin & $\begin{array}{l}\text { Spin photoresist (SPR220-3.0, } \\
\text { 4000rpm, 60sec) }\end{array}$ \\
\hline REL & Bake & SPR220 & HotPlate & Pre-Bake $\left(115^{\circ} \mathrm{C}, 90 \mathrm{sec}\right)$ \\
\hline REL & Expose & REL & AS200 & REL exposure $\left(40 \mathrm{~mJ} / \mathrm{cm}^{2}\right)$ \\
\hline REL & Bake & SPR220 & HotPlate & $\begin{array}{l}\text { Post-exposure bake }\left(115^{\circ} \mathrm{C} \text {, }\right. \\
\text { 90sec) }\end{array}$ \\
\hline REL & Develop & MIF300 & Hamatech & $\begin{array}{l}\text { Develop in MIF-300, } 120 \mathrm{sec} \\
\text { DP }\end{array}$ \\
\hline REL & Descum & SPR220 & Ox80 & $\begin{array}{l}\text { REL resist descum (30mT, } \\
\left.100 \mathrm{~W}, 50 \mathrm{sccm}_{2}, 2 \mathrm{~min}\right)\end{array}$ \\
\hline REL & Cleave & Wafer & Scribe & $\begin{array}{l}\text { Hand-cleave wafer to singu- } \\
\text { late dies }\end{array}$ \\
\hline REL & Etch & $\overline{\mathrm{SiO}_{2}}$ & Wet & $\begin{array}{l}\text { BOX sacrificial etch (BOE 6:1, } \\
\text { 5.5min) }\end{array}$ \\
\hline REL & Rinse & DI & Wet & DI water soak (4 baths) \\
\hline REL & Rinse & Acetone & Wet & $\begin{array}{l}\text { Acetone soak to remove REL } \\
\text { SPR220-3.0 }\end{array}$ \\
\hline REL & Rinse & Methanol & Wet & $\begin{array}{l}\text { Soaks in } 75 \% / 25 \%, 50 \% \\
/ 50 \%, 25 \% / 75 \% \text { Ace- } \\
\text { tone/Methanol, then } 100 \% \\
\text { Methanol. }\end{array}$ \\
\hline REL & CPD & $\mathrm{CO}_{2}$ & Tousimis & $\begin{array}{l}\text { Critical Point Dry, 30min } \\
\text { purge. }\end{array}$ \\
\hline
\end{tabular}




\section{BIBLIOGRAPHY}

[1] W. C. Tang, T.-C. H. Nguyen, and R. T. Howe. Laterally driven polysilicon resonant microstructures. In Proceedings of IEEE Micro Electro Mechanical Systems, pages 53-59, 1989.

[2] J. R. Clark, W.-T. Hsu, and C. T.-C. Nguyen. High-q vhf micromechanical contour-mode disk resonator. In IEEE International Electron Device Meeting, Technical Digest, pages 493-496, 2000.

[3] S. Pourkamali and F. Ayazi. 18 $\mu m$ thick high frequency capacitive harpss resonators with reduced motional resistance. In Solid-State Sensor, Actuator and Microsystems Workshop (Hilton Head), pages 392-393, 2004.

[4] S. Bouwstra, F. R. Blom, T. S. J. Lammerink, H. Yntema, P. Schrap, J. H. J. Fluitman, and M. Elwenspoek. Excitation and detection of vibrations of micromechanical structures using a dielectric thin film. Sensors and Actuators, 17:219-223, 1989.

[5] S. A. Bhave and R. T. Howe. Internal electrostatic transduciton for bulkmode mems resonators. In Solid-State Sensor, Actuator and Microsystems Workshop (Hilton Head), pages 59-60, 2004.

[6] Y.-W. Lin, S.-S. Li, Y. Xie, Z. Ren, and C. T.-C. Nguyen. Vibrating micromechanical resonators with solid dielectric capacitive transducer gaps. In Proceedings of IEEE International Frequency Control Symposium, pages 128-134, 2005.

[7] S. A. Bhave and R. T. Howe. Silicon nitride-on-silicon bar resonator using internal electrostatic transduction. In IEEE Solid-State Sensors, Actuators and Microsystems (Transducers), Technical Digest, pages 2139-2142, 2005.

[8] H. Chandrahalim, D. Weinstein, and S. A. Bhave. Thickness shear mode vibrations in silicon bar resonators. In Proceedings of IEEE Ultrasonics Symposium, pages 898-901, 2005.

[9] L.-W. Hung, C. T.-C. Nguyen, Y. Xie, Y.-W. Lin, S.-S. Li, and Z. Ren. Uhf micromechanical compound- $(2,4)$ mode ring resonators with solid-gap transducers. In Proceedings of IEEE International Frequency Control Symposium, pages 1370-1375, 2007. 
[10] S. A. Bhave L. F. Cheow, H. Chandrahalim. Mems filter with voltagetunable center frequency and bandwidth. In Solid-State Sensor, Actuator and Microsystems Workshop (Hilton Head), pages 304-307, 2006.

[11] S. Pourkamali, G. K. Ho, and F. Ayazi. Vertical capacitive sibars. In Proceedings of IEEE Micro Electro Mechanical Systems, pages 211-214, 2005.

[12] G. Piazza, P. J. Stephanou, J. M. Porter, M. B. J. Wijesundara, and A. P. Pisano. Low motional resistance ring-shaped contour-mode aluminum nitride piezoelectric micromechanical resonators for uhf applications. In Proceedings of IEEE Micro Electro Mechanical Systems, pages 20-23, 2005.

[13] F.P. Stratton, D. T. Chang, D. J. Kirby, R. J. Joyce, T.-Y. Hsu, R. L. Kubena, and Y.-K. Yong. A mems-based quartz resonator technology for ghz applications. In Proceedings of IEEE International Frequency Control Symposium, pages 27-34, 2004.

[14] T. Mattila, A. Oja, H. Seppa, O. Jaakkola, J. Kiihamaki, H. Kattelus, M. Koskenvuori, P. Rantakari, and I. Tittonen. Micromechanical bulk acoustic wave resonator. In Proceedings of IEEE Ultrasonics Symposium, pages 945-948, 2002.

[15] R. D. Blevins. Formulas for natural frequency and mode shape. Krieger Publishing Co., 1979. p. 176.

[16] R. Aigner. High performance rf-filters suitable for above ic integration: film bulk-acoustic-resonators (fbar) on silicon. In Proceedings of IEEE Custom Integrated Circuits Conference, pages 141-146, 2003.

[17] R. C. Ruby, A. Barfknecht, C. Han, Y. Desai, F. Geefay, G. Gan, M. Gat, and T. Verhoeven. High-q fbar filters in a wafer-level chip-scale package. In IEEE International Solid-State Circuits Conference, Technical Digest, pages 184-185, 2002.

[18] G. G. Fattinger, J. Kaitila, R. Aigner, and W. Nessler. Single-to-balanced filters for mobile phones using coupled resonator baw technology. In Proceedings of IEEE Ultrasonics Symposium, pages 416-419, 2004.

[19] J. D. Larson and R. C. Ruby. Thin-film acoustically-coupled transformer. US Patent 6946928, September 2005.

[20] S.-S. Li, Y.-W. Lin, Z. Ren, and C. T.-C. Nguyen. Self-switching vibrating 
micromechanical filter bank. In Proceedings of IEEE International Frequency Control Symposium, 2005.

[21] Y. Xie, S.-S. Li, Y.-W. Lin, Z. Ren, and C. T.-C. Nguyen. Uhf micromechanical extensional wine-glass mode ring resonators. In IEEE International Electron Device Meeting, Technical Digest, pages 39.2.1-39.2.4, 2003.

[22] J. R. Clark, M. Pai, B. Wissman, G. He, and W.-T. Hsu. Parallel-coupled square-resonator micromechanical filter arrays. In Proceedings of IEEE International Frequency Control Symposium, pages 485-490, 2006.

[23] P. J. Stephanou, G. Piazza, C. D. White, M. B. J. Wijesundara, and A. P. Pisano. Mechanically coupled contour mode piezoelectric aluminum nitride mems filters. In Proceedings of IEEE Micro Electro Mechanical Systems, pages 906-909, 2006.

[24] K. Wang and C. T.-C. Nguyen. High-order medium frequency micromechanical electronic filters. Journal of Microelectromechanical Systems, 8(4):534-557, 1999.

[25] M. P. Castanier and C. Pierre. Individual and interactive mechanisms for localization and dissipation in a mono-coupled nearly-periodic structure. Journal of Sound and Vibration, 168(3):479-505, 1993.

[26] J. A. Judge, B. H. Houston, D. M. Photiadis, and P. C. Herdic. Effects of disorder in one and two dimensional micromechanical resonator arrays for filtering. Journal of Sound and Vibration, 290(3-5):953-956, 2006.

[27] B. A. Auld. Acoustic Fields and Waves in Solids. Kreiger Publishing Company, 2nd edition edition, 1990.

[28] V. Kaajakari, A. T. Alastalo, and T. Mattila. Electrostatic transducers for micromechanical resonators: free space and solid dielectric. IEEE Transactions of Ultrasonics, Ferroelectrics, and Frequency Control, 2006.

[29] S. Gevorgian, A. Vorobiev, and T. Lewin. Dc field and temperature dependent acoustic resonances in parallel-plate capacitors based on $\mathrm{srtio}_{3}$ and $\mathrm{ba}_{0.25} \mathrm{Sr}_{0.75} \mathrm{tio}_{3}$ films: experiment and modeling. Journal of Applied Physics, 99(124112), 2006.

[30] B. Bircumshaw, G. Liu, H. Takeuchi, T.-J. King, R. T. Howe, O. O'Reilly, and A. Pisano. The radial bulk annular resonator: toward a $50 \mathrm{ohm}$ rf mems 
filter. In IEEE Solid-State Sensors, Actuators and Microsystems (Transducers), Technical Digest, pages 875-878, 2003.

[31] R. E. Newnham, V. Sundar, R. Yimnirun, J. Su, and Q. M. Zhang. Electrostriction: nonlinear electromechanical coupling in solid dielectrics. Journal of Physical Chemistry B, 101(48):10141-10150, November 1997.

[32] A.-C. Wong and C. T.-C. Nguyen. Micromechanical mixer-filters ('mixlers'). Journal of Microelectromechanical Systems, 13(1):100-112, 2004.

[33] J. T. M. van Beek, G. J. A. M. Verheijden, G. E. J. Koops, K. L. Phan, C. van der Avoort, J. van Wingerden, and J. J. M. Bontemps D. E. Badaroglu. Scalable $1.1 \mathrm{ghz}$ fundamental mode piezoresistive silicon mems resonator. In IEEE International Electron Device Meeting, Technical Digest, pages 411414, 2007.

[34] H.C. Nathanson, W.E. Newell, R.A. Wickstrom, and J.R. Davis Jr. The resonant gate transistor. IEEE Transactions on Electron Devices, 14:117-133, 1967.

[35] T. B. Gabrielson. Mechanical-thermal noise in micromachined acoustic and vibration sensors. IEEE Transactions on Electron Devices, 40(5):903-909, 1993.

[36] F. N. Hooge and L. K. J. Vandamme. Lattice scattering causes $1 / \mathrm{f}$ noise. Physics Letters A, 66(4):315-316, 1978.

[37] J. W. M. Rogers and C. Platt. Radio Frequency Integrated Circuit Design. Artech House, 2003.

[38] D. M. Fried. The design, fabrication, and characterization of independent-gate FinFETs. PhD thesis, Cornell University, May 2004.

[39] M.-H Cho, G.-W. Huang, C.-S. Chiu, K.-H. Chen, A.-S. Peng, and Y.-M. Teng. A cascade open-short-thru (cost) de-embedding method for microwave on-wafer characterization and automatic measurement. IEICE Transactions on Electronics, E88-C(5):845-850, 2005. 